\title{
Tangible heart, silicon brain
}

Citation for published version (APA):

Jin, H. (2021). Tangible heart, silicon brain: computational modelling of cardiovascular diseases. [Doctoral Thesis, Maastricht University]. Ridderprint. https://doi.org/10.26481/dis.20210921hj

Document status and date:

Published: 01/01/2021

DOI:

10.26481/dis.20210921hj

Document Version:

Publisher's PDF, also known as Version of record

\section{Please check the document version of this publication:}

- A submitted manuscript is the version of the article upon submission and before peer-review. There can be important differences between the submitted version and the official published version of record.

People interested in the research are advised to contact the author for the final version of the publication, or visit the DOI to the publisher's website.

- The final author version and the galley proof are versions of the publication after peer review.

- The final published version features the final layout of the paper including the volume, issue and page numbers.

Link to publication

\footnotetext{
General rights rights.

- You may freely distribute the URL identifying the publication in the public portal. please follow below link for the End User Agreement:

www.umlib.nl/taverne-license

Take down policy

If you believe that this document breaches copyright please contact us at:

repository@maastrichtuniversity.nl

providing details and we will investigate your claim.
}

Copyright and moral rights for the publications made accessible in the public portal are retained by the authors and/or other copyright owners and it is a condition of accessing publications that users recognise and abide by the legal requirements associated with these

- Users may download and print one copy of any publication from the public portal for the purpose of private study or research.

- You may not further distribute the material or use it for any profit-making activity or commercial gain

If the publication is distributed under the terms of Article $25 \mathrm{fa}$ of the Dutch Copyright Act, indicated by the "Taverne" license above, 


\section{Tangible Heart, Silicon Brain}

Computational Modelling of Cardiovascular Diseases 
(C) Han Jin, Maastricht 2021

All rights reserved. No part of this book may be reproduced, stored in a retrieval system of any nature, or transmitted in any form by any means (electronic, mechanical, photocopying, recording or otherwise) without prior written permission of the author, or when appropriate, by the publishers of the publications.

ISBN

Author

Layout and figure design

Cover design

Printed by
978-94-6416-717-7

Han Jin

Han Jin

Wen Li

Ridderprint | www.ridderprint.n|

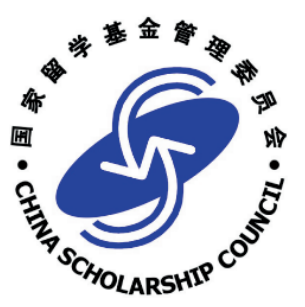

The research presented in this thesis was conducted at the Department of Pathology, Maastricht University Medical Center+ (MUMC+). The author is financially supported by the China Scholarship Council (CSC, No.201609120004). 


\title{
Tangible Heart, Silicon Brain \\ Computational Modelling of Cardiovascular Diseases
}

\author{
Dissertation \\ to obtain the degree of Doctor at Maastricht University, \\ on the authority of the Rector Magnificus, \\ Prof. dr. Rianne M. Letschert, \\ in accordance with the decision of the Board of Deans, \\ to be defended in public on
}

Tuesday, $21^{\text {st }}$ of September 2021, at 13.00 hours

by

Han Jin 


\section{Supervisors}

Prof. dr. Erik A.L. Biessen

Prof. dr. Judith C. Sluimer

\section{Co-supervisor}

Dr. Evgueni Smirnov

\section{Assessment committee}

Prof. dr. Ilja Arts (Chair)

Prof. dr. Menno de Winther (University of Amsterdam, the Netherlands)

Prof. dr. Yvan Saeys (Ghent University, Belgium)

Dr. S.L.M. Steinbusch - Coort 


\section{TABLE OF CONTENTS}

Chapter 1 General introduction

Chapter 2 Identification of a PRDM1-driven gene network to regulate $T$ cell activity in high-risk human plaque

Chapter 3 Integrative multi-omics analysis of human atherosclerosis reveals a serum response factor-driven network associated with intraplaque haemorrhage

Chapter 4 Genome-scale metabolic modelling of human carotid plaque reveals the pivotal role of glutamine/glutamate metabolism in plaque inflammation and vulnerability

Chapter 5 Towards a function-based model of macrophage plasticity: the MacroScreen platform

Chapter 6 Blood milieu in acute myocardial infarction reprograms 179 human macrophages for trauma repair

Chapter 7 Transcriptional sex dimorphism in human atherosclerosis relates to plaque type

Chapter 8 General discussion

Chapter 9 Summary | Samenvatting | 总结

Chapter 10 Impact 255

Appendices List of abbreviations

265

Acknowledgements | 致谢

273

Curriculum Vitae 



\section{Chapter 1}

General introduction 



\section{Cardiovascular disease}

Cardiovascular diseases (CVDs) are the leading cause of death globally. According to the World Health Organization (WHO) report, an estimated 17.9 million people died from CVDs in 2016, representing 31\% of all deaths worldwide ${ }^{1}$. More than $75 \%$ of CVD deaths occur in low- and middle-income countries. In China, the prevalence and mortality of CVD are continuously rising. It is estimated that in 2019, about 330 million Chinese patients suffer from CVD, and over $40 \%$ of all deaths nationwide are CVD-related, which is more than mortality due to cancer or any other diseases ${ }^{2}$. Besides, CVDs vastly increase public healthcare expenditures in China as well as worldwide. The fast increase of CVDs brings severe challenges to public health and urges for strong efforts to control it.

Of all CVDs, the two most common and lethal events, making up 85\% of CVD deaths, are heart attack and stroke. These are mainly caused by coronary (CAD) and carotid artery disease, respectively. According to statistics from the American Heart Association (AHA), CVDs present in about $50 \%$ of United States adults, including young people ${ }^{3}$. At early disease stages, people generally do not have symptoms, and only until decades later, the exacerbation of the disease progression causes acute cardiovascular events and becomes a serious health risk to cardiovascular disease patients. Atherosclerosis accounts for the acute events of the aforementioned CVDs. The presence of atherosclerotic plaques in the large arteries narrows the lumen and leads to abnormal blood flow. Furthermore, end-stage plaques may rupture or erode, and consequently, a thrombus may block the arteries. This will prevent blood from flowing to the heart and brain, leading to ischemic events, such as myocardial infarction (MI) and stroke.

\section{Atherosclerosis and macrophages}

A healthy artery is usually composed of three layers: the intima, media and adventitia. The intima layer consists of endothelial cells (ECs) lining the lumen of the vessel, as well as a subendothelial layer lying immediately under the intimal endothelium. Outside the intima is the media, which is primarily made up of smooth muscle cells (SMCs). The adventitia is the outermost layer of the artery and is mainly composed of collagen, loose connective tissue, and small blood vessels (vasa vasorum), which provide oxygen and nutrients to the medial and adventitial layers of arteries.

Atherosclerosis is a chronic, inflammatory disease characterized by the accumulation of lipids, immune cells and fibrous tissue in the inner lining of the arteries ${ }^{4}$. It is initiated by the entry and subendothelial retention of plasma apolipoprotein-B-containing lipoproteins (apoB LPs) into the arterial wall, under the agency of a variety of stimuli, including shear stress, hypertension, dyslipidaemia, 
and hyperglycaemia ${ }^{5}$. Subendothelial apoB LPs, such as low-density lipoprotein $(L D L)$, trigger an innate immune response after oxidative modification (oxLDL) ${ }^{6}$. This results in the recruitment of circulating monocytes entering into the intimal space, followed by the differentiation of circulating monocytes into macrophages of different phenotypes?

Macrophages aim at cleaning the intima from oxidized, cholesterol-laden lipoproteins ${ }^{8}$. In general, macrophages can be differentiated into two main phenotypes: pro-inflammatory (classical, M1) macrophages which can start and sustain inflammatory responses; and anti-inflammatory (alternative, M2) macrophages for tissue repairment ${ }^{9}$. Classical (M1) macrophages can be polarized from monocytes by treatment with pro-inflammatory agents like lipopolysaccharide (LPS) or interferon-gamma (IFN- $\gamma$ ), whereas alternative (M2) macrophages can be polarized by treatment with interleukin-4 (IL-4). In general, pro-inflammatory macrophages promote atherosclerosis progression and amplify local inflammation by secreting chemotactic cytokines and producing reactive oxygen species (ROS) after the formation of lipid-laden macrophages, called foam cells, while ingesting cholesterol particles (lipid uptake). The primary function of the anti-inflammatory macrophages is to dampen inflammation and clear the apoptotic cells (efferocytosis) from the accumulation of foam cells due to lipid overload and to prevent post-apoptotic necrosis ${ }^{10}$.

Macrophage phenotype both influences and is influenced by adaptive immune cell responses, mediated amongst others by cytotoxic $\mathrm{CD} 8^{+} \mathrm{T}$ cells, helper $\mathrm{CD} 4^{+} \mathrm{T}$ cells $(T h 1, T h 2, T h 17, \ldots)$ and regulatory $\mathrm{CD}^{+} \mathrm{T}$ cells $(\mathrm{Treg})^{11}$. Both Th and Treg cells migrate into progressing atherosclerotic lesions, modulating the local microenvironment. Th1 is the main subpopulation of the helper T cells in atherosclerosis, stimulating atherogenesis by secreting the signature cytokine IFN$\gamma$ to sustain the inflammatory response in the intima. In contrast, Treg cells, which decrease during the development of atherosclerosis, exert immunosuppressive actions by producing anti-atherogenic cytokines such as IL-4, IL-5, IL-10 and IL-13 and transforming growth factor- $\beta$ (TGF- $\beta)^{12}$. However, the role of many T cell subpopulations including $\mathrm{CD} 8^{+} \mathrm{T}$ cell subsets in the development of atherosclerosis is still controversial ${ }^{13}$.

The balance between $\mathrm{M} 1$ and $\mathrm{M} 2$ macrophages is thought to actively influence atherosclerosis progression. The uptake of excessive cholesterol particles by M1 macrophages will induce the abundant formation of lipid-laden macrophages, as known as foam cells, which not only augment the recruitment of circulating monocytes but also show defective efferocytosis. The latter leads to the accumulation of non-cleared apoptotic cells, expanding the necrotic core, and leading to plaque progression into a more advanced stage ${ }^{14}$. 
Upon vascular injury, vascular smooth muscle cells (VSMCs) in the media will start proliferating and will migrate from the media towards the intima. These VSMCs will produce and secrete large amounts of extracellular matrix components (e.g. collagen, elastin and proteoglycans) and form a fibrous cap that covers the necrotic core $^{15}$. This extracellular matrix stabilizes the plaque and prevents advanced plaques from rupture. However, as discussed above, phagocytic clearance of apoptotic cells is no longer efficient in advanced plaque, and the apoptotic cells will die by pro-inflammatory and radical producing necrosis, further boosting the hypoxic and inflammatory environment in atherosclerotic plaque. Macrophages secreting matric metalloproteinases stimulated degradation of the fibrous cap. Also, the prevailing hypoxia will drive microvessel growth, from the vasa vasorum into the plaque (angiogenesis). These intraplaque microvessels, however, lack a continuous basement membrane and are leaky, as a result of poorly formed tight junctions. This results in intraplaque haemorrhage (IPH), the influx of more inflammatory cells, and iron- and cholesterol-rich erythrocytes, together making the plaque susceptible to rupture ${ }^{16}$.

As the disease progresses, the fibrous cap becomes thinner and will eventually rupture $^{17-19}$. Plaque rupture mostly happens at the biomechanically weakest spot like the cap margin or shoulder region, accompanied by necrotic core leakage, angiogenesis, and intraplaque haemorrhage $\mathrm{e}^{20}$. The thrombotic complication occurs when the highly thrombogenic necrotic core is exposed to the coagulation proteins in the blood. Finally, the formation of thrombosis blocks the artery, leading to acute clinical symptoms such as myocardial infarction and stroke (Figure 1).

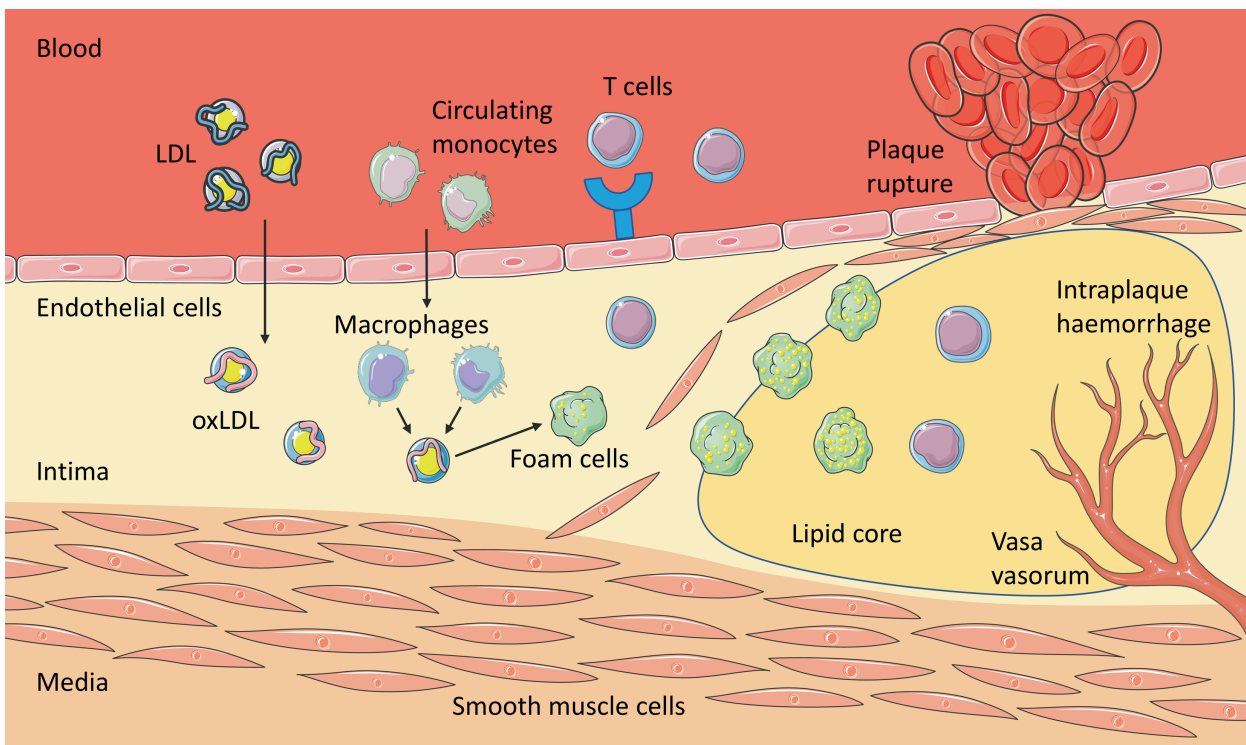

Figure 1. The development of atherosclerosis (adapted from Ruparelia et al. ${ }^{7}$ ) 


\section{Classification of atherosclerotic lesions}

For the studies on atherogenesis, it is important to precisely define and describe the disease progression from early to late stage. Virmani et al. modified the AHA classification initially defined by Stary et al. ${ }^{21}$ into a simplified plaque classification scheme based on histological characteristics ${ }^{17,18}$. Previous studies in our research group classified carotid plaques into three main groups: early, advanced stable, and ruptured lesions. Early lesions are plaques with features of "intimal thickening", "intimal xanthoma" and "pathologic intimal thickening", which is similar to that reported by Stary et $\mathrm{al}^{21}$. Advanced stable lesions can be characterized by the formation of the fibrous cap covering the necrotic core heavily infiltrated by macrophages and T cells (thick cap fibroatheroma). Ruptured plaques are defined by the disruption of the fibrous cap with the thrombus on top of the necrotic core. However, few plaques harbour signs of rupture when stained for Haematoxylin and Eosin (H\&E). Therefore we use a surrogate feature of plaque rupture, which is the presence of intraplaque haemorrhage ${ }^{22}$. Several studies suggested IPH as a sign of cracks or fissures originating from the luminal surface and a common feature for plaque rupture and luminal thrombi ${ }^{20,23}$.

In chapter 2, 3, 4 and $\mathbf{7}$ of this thesis, we mainly rely on the Maastricht Human Plaque Study (MaasHPS) cohort $^{24}$ to study the differences between stable, low-risk and rupture-prone, high-risk plaques. This cohort includes a total of 43 atherosclerotic lesions from patients undergoing carotid endarterectomy (CEA) surgery. In this cohort, plaques were categorized into two groups based on the presence or absence of IPH, resulting in 16 non-IPH and 27 IPH plaques. Non-IPH plaques are mostly early and advanced lesions, regardless of the thickness of the fibrous cap. The IPH group involves rupture-prone and ruptured plaques.

\section{Macrophage in Post-infarct repair}

Apart from being crucial in the progression of atherosclerosis, macrophages also play important roles in other cardiovascular-associated inflammation processes. For example, in post-MI repairment, the occurrence of acute myocardial infarction (AMI) directly changes the blood composition and affects the circulating system by raising an inflammatory injury/repair response through the whole body ${ }^{25,26}$. Following $\mathrm{Ml}$, circulating monocytes are recruited and differentiated into adverse phenotypes of macrophages, helping the cardiac resident macrophages with cardiac repairment ${ }^{27}$. Similar to the two distinct functions of $M 1$ and $M 2$ macrophages in atherosclerosis, in the early post-MI stage, cardiac macrophages play a pro-inflammatory role by secreting pro-inflammatory cytokines ${ }^{28}$, whereas in the later post-MI stage, the function of macrophages is shifted towards a more pro-repair phenotype. However, the activation and function of macrophages in the $\mathrm{AMI}$ environment remain unclear.

Page | 12 
In 2016, a study proposed a more complex multispectral model of macrophage activation ${ }^{29}$. Instead of the 2-stage model of monocyte-derived macrophage polarization into two extremely distinct phenotypes: the pro-inflammatory cytokine IFN- $\gamma$ (M1) and anti-inflammatory cytokine IL-4 (M2), Xue et al. activated macrophages by a variety of 28 stimuli, and further grouped the 28 conditions with control into nine main categories based on gene expression profiles. Each phenogroup was characterized by a shared transcriptional signature, and differentiation was both driven by transcription factors and epigenetic control. This study highlighted the high plasticity and complexity of macrophage activation, and showed the well adaptable of macrophage activation and polarization to the microenvironment.

\section{High-throughput analysis in cardiovascular disease}

The rapid development and wide application of high-throughput techniques, such as genomics, transcriptomics, epigenomics, proteomics, metabolomics and lipidomics marked a new era for the cardiovascular research field. While traditional wet lab-based experimental methodologies merely focus on one or several key variates to the disease, high-throughput profiling enables to grasp global changes in expression and explore the relevant pathways instead of a single molecule that promotes the disease progression. Furthermore, single-cell RNA sequencing (scRNA-seq) as well as cellular indexing of transcriptomes and epitopes by sequencing (CITE-seq) offer the possibility to measure gene expression and quantitative/qualitative information on surface proteins at a single-cell level ${ }^{30}$. To date, more and more research groups utilize the advantages of high-throughput analysis to study cardiovascular diseases.

Many human atherosclerotic plaque cohorts have been built up to study carotid atherosclerosis and related cardiovascular diseases using high-throughput techniques. The Biobank of Karolinska Endarterectomy (BiKE) is a well-known CEA biobank including microarray gene expression profiles of CEA samples from carotid plaques and peripheral blood mononuclear cells (PBMCs) from hundreds of symptomatic and asymptomatic patients ${ }^{31,32}$. Another famous biobank for the atherosclerotic study is the Athero-Express ${ }^{33}$, an ongoing longitudinal study consisting of genomics and proteomics, linked to extensive clinical phenotyping from patients undergoing CEA and femoral endarterectomy (FEA). Plaque composition-based risk factors used in this thesis, such as IPH, macrophage infiltration, collagen content and calcification, have been studied using survival analysis on the Athero-express cohort ${ }^{34}$. Besides, the Stockholm-Tartu Atherosclerosis Reverse Networks Engineering Tasks (STARNET), provide various biological networks inference through thousands of RNA expression profiles from multiple cardiovascular disease-relevant tissues from 600 coronary artery bypass 
grafting patients ${ }^{35}$. On proteomics level, Mayr and his colleagues have extensively applied Mass Spectrometry (MS)-based technology to measure the protein abundance and integrate proteomics with transcriptomics and metabolomics to study the biological pathways in cardiovascular disease ${ }^{36}$. On metabolomics level, a recent study in 2018 measured 165 different metabolites from 159 carotid plaques of CEA patients and identified a metabolite profile that was associated with plaque instability, vascular inflammation and presence of symptoms ${ }^{37}$. Despite the fact that these studies only analysed plaque omics profiling on the bulk tissue level, and thus were not able to identify the cellular source of the altered molecules and pathways, they substantially showed the power of applying high-throughput analysis in atherosclerosis and extensively broadened the cardiovascular researchers' landscape and brought profound knowledge to cardiovascular research.

A next, significant step forward was the development and application of single-cell sequencing on atherosclerotic plaque study ${ }^{38}$. Cell composition of carotid atherosclerotic plaques is rather complex and highly site, stage and patient dependent - an advanced plaque usually involves infiltrated macrophages, T cells, SMCs, ECs, fibroblasts, and so on. Each cell type can be subdivided into several subpopulations with distinct biological functions by different signatures and cell stages. Standard bulk omics technologies, such as microarrays and bulk RNA-seq, can only assess the average expression/abundance of the cell population mixture in plaque tissue. Single-cell sequencing overcomes this limitation. With the ability to measure RNA expression on the single-cell level, scRNA-seq can evaluate gene expression within a specific cell population. Furthermore, it can be also used to discover novel cell subpopulations within a heterogeneous cell population ${ }^{39}$, which is often observed in immune-infiltrated tumours and atherosclerotic plaques. In the recent three years, many remarkable applications of single-cell sequencing on atherosclerosis have been carried out. In 2019, Wirka et al. published a study using scRNA-seq on both mouse and human plaques followed by a canonical correlationbased analysis and discovered TCF21 as an SMC phenotypic modulation that contributes to the formation of protective fibrous cap of the lesions $\mathrm{s}^{40}$. In the same year, Fernandez et al. created a single-cell immune landscape of human CEA plaque and defined the cell-cell interaction between plaque T cells and macrophages by applying mass cytometry (CyTOF), CITE-seq and scRNA-seq on human plaque lesions from both symptomatic and asymptomatic patients ${ }^{41}$. In 2020, a comprehensive cellular atlas of human carotid atherosclerotic plaque was created by Pasterkamp et al. using single-cell RNA and single-cell ATAC sequencing ${ }^{42}$. Meanwhile, a meta-analysis including nine scRNA-seq datasets from mouse aortas was carried out by Zernecke et al. to study the leukocyte diversity in atherosclerosis ${ }^{43}$. It is foreseeable that more and more studies on atherosclerotic plaques will utilize the advantages of high-throughput and single-cell screening, Page | 14 
which will lead to substantial breakthroughs in cardiovascular research.

\section{The traditional (transcript)omics data analysis pipeline}

The traditional pipeline for high-throughput gene expression data analysis consists of statistically-based gene differential expression analysis, followed by overrepresentation and pathway analysis on the differentially expressed genes $(D E G s)^{44}$. Using statistical testing, researchers can observe whether a given gene is significantly differentially expressed in one condition over the other. It can be also used to discover all significant DEGs that are triggered by disease-inherent mechanisms versus healthy tissue. To achieve statistical significance, a minimum of three replicates is generally required for differential expression analysis, which means for a case-control study, a batch of transcriptional profiles of six samples is sufficient. Thus, the cost of performing a traditional high-throughput gene expression experiment is by now, acceptable, making the traditional analytical workflow flexible and applicable to many studies.

Having obtained the set of DEGs, gene set overrepresentation analysis (GSOA) and pathway analysis can be used to define the relevant biological processes and pathways that are associated with the DEG set. These are usually statistically-based methods using Fisher's exact test based on the hypergeometric distribution. Applying such analyses requires a set of query genes that are up-/down-regulated by the disease or intervention, or a set of genes that are inferred to be co-functional in a certain condition. By comparing the overlap between query genes and preannotated genes, conclusions can be made on whether a specific biological process or pathway is significantly associated with the query genes. In this way, the most associated pathways and biological processes to the set of DEGs can be revealed. Many public available databases for gene functional annotation and pathways, such as Gene Ontology $(\mathrm{GO})^{45}$, Kyoto Encyclopedia of Genes and Genomes (KEGG) ${ }^{46}$, Reactome Pathway Database ${ }^{47}$, and Wikipathways ${ }^{48}$, are widely used for GSOA. This traditional analysis pipeline has been widely applied on transcriptomics with microarray or bulk RNA-seq data, as well as on scRNA-seq data.

Despite the outstanding performance of the traditional workflow for analysing omics data, it has several limitations. First, while screening significant DEGs from more than ten thousand genes, the sensitivity and the discovery of false-positive DEGs needs to be controlled and balanced carefully ${ }^{49}$. This is likely to happen when the minimal requirement of sample size (three per group) is used for analysing tissues with high inter-individual differences such as human plaque. Increasing the number of replicate samples can significantly improve the detection power, however, this may lead to an extra cost. Second, GSOA based on screened DEGs can reveal all relevant biological pathways altered by the disease, but pathways with corresponding functional genes are not well organized and need to be further 
clarified by more advanced models. Furthermore, mutual interactions between altered genes or pathways are not accounted for by the traditional workflow. Also, DEG-based statistical approaches generally perform poorly for designing disease or treatment classifiers ${ }^{50}$. Therefore, more sophisticated methods are required for the analysis of high-throughput data.

\section{Machine Learning and its application in Bioinformatics}

With the rapid generation of high-throughput data, a lot of bioinformatic approaches, including machine learning-based strategies, have been developed ${ }^{51}$. Machine learning can be roughly divided into two categories: supervised and unsupervised learning. Supervised learning aims at training a prediction model based on sample data with certain labels to make predictions for unknown samples, and unsupervised learning aims at clustering unlabelled samples into several groups (Figure 2).
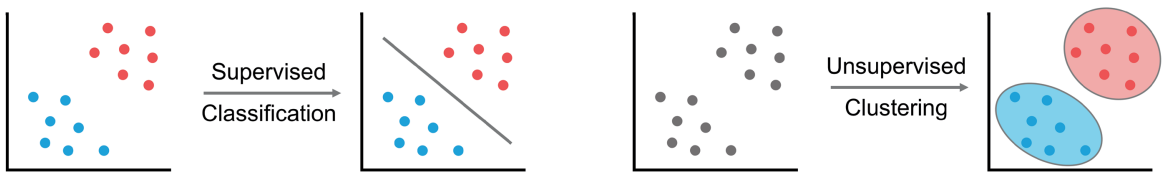

Figure 2. Supervised and unsupervised learning

Supervised learning aims at learning a decision function from labelled samples to classify unlabelled samples. Unsupervised learning looks for the sample relationships and undetected patterns from unlabelled data.

Applying cutting-edge supervised machine learning algorithms on biological data has led to breakthroughs in biomedical diagnosis ${ }^{52}$. For example, in 2014, Google proposed a deep convolutional neural network called "GoogLeNet" that can diagnose cancer from images faster than pathologists ${ }^{53}$. The accuracy of GoogLeNet was around $89 \%$, which is significantly superior to the accuracy of $73 \%$ achieved by a team of human pathologists. Despite this success, we note a well-known limitation of the machine learning algorithms: they often overfit the training data; i.e. they produce prediction models that eminently fit the training data and may fail to predict new samples ${ }^{54}$. This problem is well present for omics data in general, especially, where the dimensionality of the data (thousands to more than ten thousand) is generally much higher than the sample size (few to hundreds) ${ }^{54}$. The effect of overfitting can be diminished by controlling the data fit of the prediction models. In this context, multiple techniques have been proposed, such as regularization, ensembles, early stopping, and feature selection, that employ 
usually a form of model selection (e.g., using cross-validation) ${ }^{54}$. Among these techniques, feature selection deserves special attention due to its intrinsic ability to (partly) explain the data and prediction models $\mathrm{s}^{55}$. By definition, feature selection reduces the number of features, and thus simplifies data representation, the model, and their predictions ${ }^{56}$. It can be implemented as 1) filters that select features according to some general criteria (e.g., DEGs, correlation to the outcome); 2) wrappers that select features by maximizing the generalization performance of the prediction models (e.g., accuracy rate, ROC AUC, true positive rate, etc.); and 3) embedded methods that select features during model training (e.g., decision trees, decision rules, LASSO, etc.). While the efficiency of wrapper methods largely depends on the computational complexity of the prediction model used for feature selection, filter methods are much faster when analysing high dimensional omics data. However, there is a price, especially for univariate filter methods: they tend to select highly inter-correlated, redundant features, leading to overfitting and a non-minimal feature subset. Embedded methods seem to provide a trade-off between the filter methods and wrapper methods: they are usually fast as the filter methods, while taking into account the interaction of features, which is similar to the wrapper methods. We note that these three methods have been established as standard feature-selection methods for analysing omics data ${ }^{55}$. For example, using wrappers we can identify the best predicting genes for the diseased tissues, and these genes can be viewed as key factors for the disease under study. Thus, they can be used as novel biomarkers and potential targets for therapeutic intervention.

Unsupervised machine learning algorithms have been widely used in highthroughput omics data analysis as well ${ }^{51}$. A typical example is the hierarchical clustering of transcriptomics data followed by heatmap visualization. Clustering analysis can be done on both genes and samples. Clustered genes are often considered biologically co-functional. Therefore, a GSOA can be performed to reveal the associated biological processes and pathways of the gene cluster. For scRNA-seq data with thousands of samples, clustering analysis can separate different cell types and even discover novel subpopulations in a cell population. This is usually executed after unsupervised dimensionality reduction by principal component analysis (PCA) from ten or twenty thousand genes to a lower space defined by dozens of principal components ${ }^{57}$. PCA can significantly reduce dimensionality and remove irrelevant information and noise while minimizing the loss of principal information, which is crucial in downstream clustering analysis. However, PCA relies on an orthogonal linear transformation from the original data to a new projection space, and, thus, it has a poor performance when dealing with non-linear data. For visualization of cell subpopulations and their distribution based on single-cell expression data, t-distributed Stochastic Neighbour Embedding $(\mathrm{tSNE})^{58}$ and Uniform Manifold Approximation and Projection (UMAP) $)^{59}$ are commonly used as they are two non-linear methods suitable for this case. A 
projection of cells from high-dimensional scRNA-seq data into two dimensions provided by tSNE or UMAP can effectively and informatively exhibit the cell heterogeneity and subpopulation division.

Another challenge in analysing single-cell sequencing data is the integration of the data from multiple modalities or individuals, e.g., integrating sCRNA-seq with CITEseq as well as SCATAC-seq, or integrating scRNA-seq data from different human individuals or even jointly analysing human data with mouse data. To this end, transfer learning-based approaches have been adapted to allow integrative analysis across different modalities, individuals, or even species ${ }^{60}$. This whole workflow for single-cell sequencing data, including data integration, clustering, dimensionality reduction and visualization, is particularly useful in cancer and immune-related studies to understand the heterogeneity, diversity and dynamics of the immune microenvironment in disease tissue.

\section{Multi-omics integration}

Despite the depth of the multitude of omics datasets available, most studies involve only one single omics layer, which may limit the understanding of the precise mechanisms of cardiovascular disease. For example, not all genetic mutations screened by DNA microarray or sequencing can cause genetic disorders and diseases. Although high-throughput gene expression data can trace and monitor a wide range of disease-induced changes on a molecular level, the identified key genes are not directly translatable to detectable markers and entities for diagnostic purposes. In contrast, large-scale protein profiling analysis provides directly translatable and functional entities. However, we note that the proteomics data have limited coverage compared to transcriptomics. Integrating multiple omics can provide an overall picture of disease mechanisms and a deeper understanding of the underlying processes involved in the disease. So far, researchers have identified more than 150 CAD-associated genetic loci by genome-wide association studies $(\mathrm{GWAS})^{61}$. Integrating genomics, transcriptomics and epigenomics, including copy number variation (CNV) and single-nucleotide polymorphism (SNP), will benefit the discovery and understanding of the DNA variation and gene causality in disease manifestation. Furthermore, different omics layers provide information at different levels. For example, to extrapolate gene expression to metabolite abundance, there is a huge gap that includes transcriptional regulation, protein biosynthesis and enzyme-catalysed metabolic reaction. Integrative analysis of transcriptomics, proteomics and metabolomics can utilize the advantage of each omics layer to build a systematic and comprehensive model that better describes the disease mechanism or trajectory. Besides, considerable heterogeneity and inconsistency exist between omics layers, which might also be a fascinating path that is worth exploring. 
Although multi-omics analyses have shown remarkable performance and promising results in the identification of new diagnostics or therapeutic targets in cancer, applying such analysing strategy on CVD is not straightforward and still faces challenges. For example, a typical multi-omics analysis on CVD identified Biliverdin Reductase $\mathrm{B}$ (BLVRB) as a marker of IPH using an intersection analysis between omics on human carotid plaques and plasma from the BiKE cohort ${ }^{32}$. This study utilized multiple omics profiling on not only arterial lesions but also plasma and/or PBMCs from the circulatory system, which requires diverse high-throughput data collection and elaborate study design. However, the interactions between pathological lesions and the circulating environment are still unclear and may need more sophisticated mathematical approaches rather than a simple overlay of omics. Therefore, the development and application of multi-omics analysis strategies in CVD are necessary and important.

\section{Network medicine}

While there are many outstanding tools for omics analysis and visualization, a promising analysing strategy shown to be superior for high-throughput omics data views the biological system as a complex network that can be analysed by networkbased strategies. It can be used not only for analysing the relationships between biological elements from a single omics layer, but also for integrating molecular elements from multiple omics into a whole system. In 2011, Barabasi et al. proposed network medicine, a novel approach to understanding human disease ${ }^{62}$. In biomedical research, a network can be used to model the interactome between biological elements. Nodes or vertexes in the network can be RNA molecules, proteins, or any other biological elements; their interactions are represented by links or edges, which can be directly derived from previous experiments, or assessed mathematically by correlation or mutual information-based relationships. Having constructed the biological network, network-based analysing strategies, such as network controllability, node centrality, and shortest path-based proximity can be applied to analyse the network property and identify the key elements, which could serve as a starting point for the discovery of novel drug target.

In the following paragraphs, I will introduce several widely applicable biological networks in biomedical research. These are gene co-expression network, gene regulatory network, protein-protein interaction network, and metabolic network (Figure 3). In addition, I will provide the scale-free property, a common and important topological structure of the biological network. 


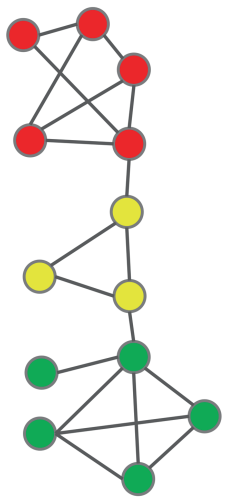

Gene co-expression network

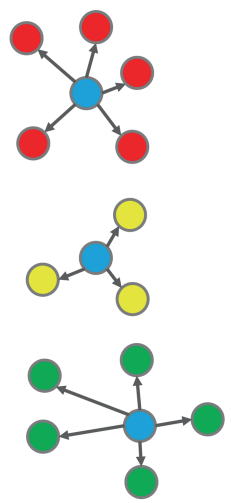

Gene regulatory network

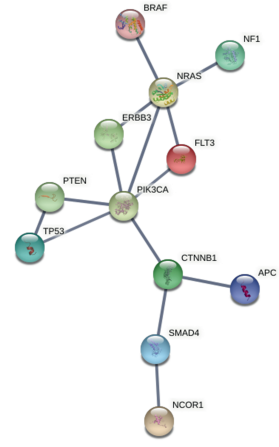

Protein-protein interaction network

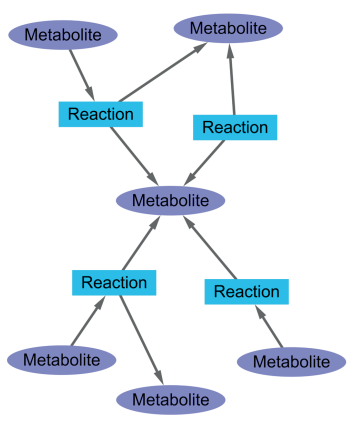

Metabolic network

Figure 3. Examples of the four types of networks used in this thesis

\section{Scale-free property}

A scale-free network is a network which the distribution $p(k)$ of node degrees $k$ follows a power law:

$$
p(k) \sim k^{-r}
$$

where $r$ is a parameter often in the range of 2 and 3. Based on this definition, the scale-free topology can be evaluated by modelling the $R^{2}$ of a linear regression between $\log _{10}(p(k))$ and $\log _{10}(k)$. Usually, a network with an $R^{2}$ above 0.8 or 0.85 is considered scale-free.

Many networks, including biological networks, are reported to be scale-free and can be modelled by scale-free topology. In a scale-free network, most of the nodes are low-degree and are interconnected via high-degree nodes called "hubs" (Figure 4). Based on this characteristic, removing nodes randomly from the network will not destroy the network structure, as most of the randomly selected nodes are not the hubs and do not substantially contribute to the network connectivity. In contrast, if the network hubs are identified and removed at the same time, the whole network will be destroyed. This is called "error tolerance", which reflects the robustness of the complex network ${ }^{63,64}$. Researchers can utilize this characteristic to analyse a biological scale-free network. Once a scale-free biological network is constructed, the key elements identified by graph-based methods can be considered as the key targets for precision medicine and intervention. 


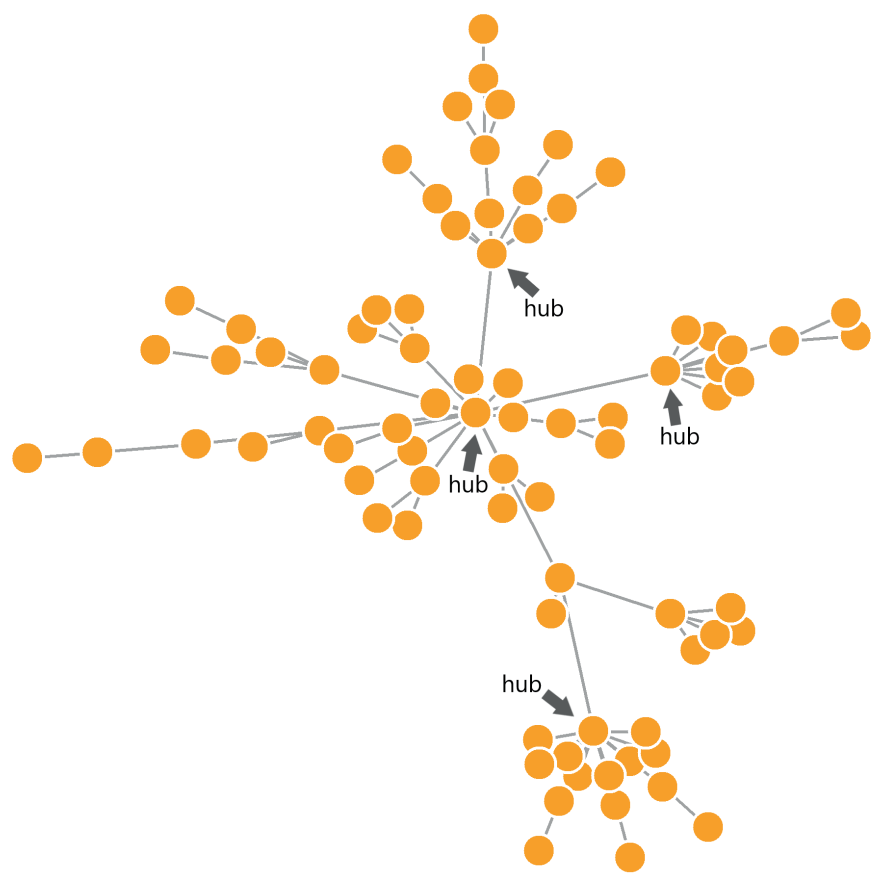

Figure 4. Example of a scale-free network

The network ( $n=80$ ) was created using the R package igraph ${ }^{65}$ (v1.2.6), following the definition of the Barabasi-Albert model under the default setting. Four nodes acting as hubs are indicated in the network.

\section{Gene co-expression network}

A gene co-expression network is an undirected network in which associated genes as nodes are linked by their co-expression relationships evaluating by Pearson's correlation coefficient (PCC) or mutual information-based similarity measurements ${ }^{66}$. Gene co-expression networks can be computationally generated from gene expression data by measuring pairwise gene similarity across a minimum of dozens of samples. After constructing the co-expression network, graph and modularity-based clustering algorithms can be deployed to detect the co-expressed genes communities/clusters. Co-expressed genes in each cluster are considered cofunctional; their biological functions and underlying molecular mechanisms relevant to the co-expressed gene clusters can be inferred by GSOA.

Many tools, such as GeneMANIA ${ }^{67}$, have been developed to study and explore gene co-expression and predict the functions of co-expressed genes. Weighted Gene Coexpression Network Analysis (WGCNA) ${ }^{68}$ is a representative framework for 
constructing and analysing gene co-expression networks. While in most cases, a scale-free co-expression network cannot be directly obtained by only measuring PCC for each gene pair, WGCNA applies a soft thresholding power $\beta$ on the PCCs to force the network to be scale-free:

$$
\alpha_{i . j}=\left|\operatorname{cor}\left(x_{i}, x_{j}\right)\right|^{\beta}
$$

where $x_{i}$ and $x_{j}$ are the expression of gene $i$ and gene $j$, and $\alpha_{i . j}$ is the transformed gene pair similarity between gene $i$ and gene $j$. After soft thresholding transformation, the scale-free topology can be approximated in most of the gene co-expression networks. Further analyses, such as gene clustering, hub gene identification and GSOA can be done on the scale-free gene co-expression network.

One of the first applications of WGCNA on CVD is made by Lusis and co-workers on the transcriptional profiles of human aortic endothelial cells ${ }^{69}$. In their study, several functional gene clusters that are associated with inflammatory responses to oxidized phospholipids in ECs from heart transplant donors, which may play important roles in atherosclerosis vulnerability ${ }^{69}$. Furthermore, despite the initial purpose of WGCNA that is designed for gene co-expression analysis, several studies showed the potential capability of applying WGCNA on proteomics, metabolomics and other omics ${ }^{70}$.

\section{Gene regulatory network}

Co-expressed genes are functionally related which may reflect the modulatory activity of a common/shared regulator ${ }^{71}$. The regulator, which is also called the "transcription factor" (TF), is a protein that binds to a specific DNA sequence to regulate the rate of transcription of a specific DNA to messenger RNA (mRNA). TFs with directed links to their regulating targets (genes) can form a gene regulatory network $(G R N)^{72}$. The identification of key TFs using network-based analysis on GRN, can provide deep insights in understanding the interactions between biological molecules, which can benefit precision medicine and the discovery of novel drugs based on drug-target interactions.

Several approaches have been developed to identify TF-target relationships. Chromatin immunoprecipitation followed by sequencing (ChIP-seq) is an experimental-based method to study the interactions between DNA and proteins ${ }^{73}$. By combining ChIP assays with sequencing, this technique can effectively detect the genome-wide DNA binding sites for TFs and other proteins. For a set of differentially expressed or co-expressed genes that are assumed to be regulated by the same TFs, the TFs can be predicted either by looking for the protein motif that can bind the common sites of the genes, or by overrepresentation analysis based on the known 
TF-target gene relationships from ChIP-seq experimental results. After that, GRN can be constructed by linking the predicted TFs with their regulating target genes.

\section{Protein-protein interaction network}

Proteins behave and act as the essential part of biological organisms. By performing a vast array of functions within organisms, proteins directly determine biological functions and control molecular and cellular mechanisms ${ }^{74}$. Proteins physically interact with each other, forming a complex system to regulate almost every process including signal transduction, catalysing metabolic reactions, responding to stimuli, and other biological activities. With the development and application of network science, proteins with their complex interactions can be logically depicted and analysed as a complex system by a network model. Protein-protein interaction (PPI) networks are usually undirected, except when depicting the relationship between kinase and its substrate. Constructing a PPI network can be based on both experimental, and computational methods.

Even though similar to the structure of the gene co-expression network, the PPI network represents the biological system at a different level. Analysing PPI networks can provide a more straightforward model/concept of the disease mechanisms. Afterwards, the network can be directly used in network-based drug screening for therapeutic intervention. Moreover, with the effect of posttranslational modifications, elements from a co-expressed gene cluster are not always highly interconnected on the protein level. Therefore, a large number of studies superimposed PPI network to co-expression network, to utilize the advantages of both networks from two different levels to form a unified biological system.

\section{Metabolic network}

The structure of a metabolic network is slightly different from the biological networks described above. A metabolic network is a directed biological network composed of metabolic substrates and products linked by metabolic reactions. Metabolic reactions are directional chemical processes that take place in living organisms and help to maintain the life cycle, including the consumption of energy for cellular processes, and the production and degradation of metabolites such as proteins, lipids, nucleic acids, and carbohydrates.

Metabolic reactions are catalysed by enzymes, i.e., the proteins that mediate chemical reactions. Considering this intrinsic feature, integrating enzyme-coding gene expression from high-throughput omics into the metabolic network can reconstruct a genome-scale metabolic model (GEM) that combines gene expression and metabolic reaction into a whole system. Furthermore, based on the GEM and gene differential expression analysis, researchers can predict the likelihood and 
significance level of the abundance of a metabolite that is directly influenced by the expression of the associated enzyme-coding genes ${ }^{75}$. So far, GEMs have been widely studied and applied in the biomedical research field to discover the metabolic dynamics and the unbalance of metabolism altered by gene expression in disease tissues. For cardiovascular research, this is particularly important, as the disarray of associated metabolism pathways is a major contributor to cardiovascular diseases and other comorbidities like obesity and diabetes. Also, for atherosclerosis study, the complex cellular composition and high diversity of cell populations in plaque implicate an intricate metabolic system in plaque formation, progression and rupture. Previous metabolic studies in cardiovascular mainly focused on the disorder of metabolites quantified by metabolomics, however, lacking a systematic overview. Therefore, a comprehensive system depicting plaque metabolism with genes, enzymes, metabolites and metabolic reactions is urgently required to understand novel mechanisms and identifying drug targets for cardiovascular diseases. 


\section{Outline of the thesis}

Summarizing all above, we postulate that the wide application of novel statistical/mathematical, machine learning- or network-based strategies in cardiovascular research will provide profound new insights into key processes in the pathogenesis of CVD, and may lead to breakthroughs in cardiovascular research. In this thesis, we therefore will focus on the application of the novel data analysis approaches to unravel high-throughput omics and integrate multiple omics data sets with the key aim first to dissect new mechanisms in the clinically relevant transition of a stable into a ruptured atherosclerotic plaque, and then to validate their relevance by wet-lab experiments and on independent data sets of CVD relevant tissue.

In chapter 2, we will construct a gene co-expression network model of atherosclerotic plaque from the MaasHPS transcriptomics dataset. Based on the constructed co-expression network, we will further extend the network analysis by a WGCNA-based Bayesian network to depict systemic processes in atherosclerotic plaque, in order to reveal the pivotal roles of T cells in plaque progression. We will also screen candidate drugs through drug repurposing to reverse the adverse function of T cells in plaque rupture.

In chapter 3, we will analyse the plaque instability by multi-omics integrative analysis consisting of transcriptomics, proteomics and peptidomics. Moreover, we will compare the performance of multi-omics to that of single-omics based models, and dissect the IPH associated regulatory factors driving this model. Chapter $\mathbf{4}$ will focus on the integration of metabolomics and transcriptomics to build an atherosclerotic plaque-specific GEM to study metabolic alterations and dysfunctions in late-stage plaque.

In chapter 5, we will develop a novel platform MacroScreen that enables measuring in-depth "functionomics" profiles of human macrophages to accelerate the biological analysis of our omics analysis findings. By integrating functionomics and transcriptomics of polarized macrophages by 28 different stimuli using canonical correlation analysis (CCA), we will establish a connection between transcriptional and functional profiles of the 28 stimulated macrophages plus control, and we will cluster them into several groups based on this shared information. The platform and data analytical pipeline will be used in chapter 6 to study and analyse the functional reprogramming of the human macrophages in patients' serum after myocardial infarction.

Chapter 7 will focus on the sex-specific transcriptional differences in human atherosclerosis. Finally, we will discuss the major findings of this thesis and the future perspectives in chapter $\mathbf{8}$. 


\section{REFERENCES}

1. WHO, https://www.who.int/news-room/fact-sheets/detail/cardiovasculardiseases-(cvds)

2. Zhao D, Liu J, Wang M, Zhang X, Zhou M. Epidemiology of cardiovascular disease in China: current features and implications. Nat Rev Cardiol. 2019;16(4):203-212.

3. Virani SS, Alonso A, Benjamin EJ, et al. Heart disease and stroke statistics2020 update: A report from the American Heart Association. Circulation. 2020;141:E139-E596.

4. Libby P, Ridker PM, Hansson GK. Progress and challenges in translating the biology of atherosclerosis. Nature. 2011;473(7347):317-325.

5. Lusis AJ. Atherosclerosis. Nature. 2000;407(6801):233-241.

6. Weber $\mathrm{C}$, Noels $\mathrm{H}$. Atherosclerosis: Current pathogenesis and therapeutic options. Nat Med. 2011;17(11):1410-1422.

7. Ruparelia N, Chai JT, Fisher EA, Choudhury RP. Inflammatory processes in cardiovascular disease: A route to targeted therapies. Nat Rev Cardiol. 2017;14(3):133-144.

8. Barrett TJ. Macrophages in Atherosclerosis Regression. Arterioscler Thromb Vasc Biol. 2020;40(1):20-33.

9. Italiani $\mathrm{P}$, Boraschi D. From monocytes to $\mathrm{M} 1 / \mathrm{M} 2$ macrophages: Phenotypical vs. functional differentiation. Front Immunol. 2014;5(OCT):514.

10. Tabas I, Lichtman AH. Monocyte-Macrophages and T Cells in Atherosclerosis. Immunity. 2017;47(4):621-634.

11. Robertson AKL, Hansson GK. T cells in atherogenesis: For better or for worse? Arterioscler Thromb Vasc Biol. 2006;26(11):2421-2432.

12. Tse K, Tse H, Sidney J, Sette A, Ley K. T cells in atherosclerosis. Int Immunol.

13. Saigusa $\mathrm{R}$, Winkels $\mathrm{H}$, Ley $\mathrm{K}$. T cell subsets and functions in atherosclerosis. Nat Rev Cardiol. 2020;17(7):387-401.

14. Tabas I. Macrophage death and defective inflammation resolution in atherosclerosis. Nat Rev Immunol. 2010;10(1):36-46.

15. Bennett MR, Sinha S, Owens GK. Vascular Smooth Muscle Cells in Atherosclerosis. Circ Res. 2016;118(4):692-702.

16. Kolodgie FD, Gold HK, Burke AP, et al. Intraplaque Hemorrhage and Progression of Coronary Atheroma. N Engl J Med. 2003;349(24):2316-2325.

17. Virmani R, Kolodgie FD, Burke AP, Farb A, Schwartz SM. Lessons From Sudden Coronary Death. Arterioscler Thromb Vasc Biol. 2000;20(5):12621275.

18. Virmani R, Burke AP, Kolodgie FD, Farb A. Vulnerable plaque: the pathology of unstable coronary lesions. J Interv Cardiol. 2002;15(6):439-446.

19. Bentzon JF, Otsuka F, Virmani R, Falk E. Mechanisms of plaque formation and rupture. Circ Res. 2014;114(12):1852-1866.

Page | 26 
20. Virmani R, Kolodgie FD, Burke AP, et al. Atherosclerotic plaque progression and vulnerability to rupture: Angiogenesis as a source of intraplaque hemorrhage. Arterioscler Thromb Vasc Biol. 2005;25(10):2054-2061.

21. Stary HC, Chandler AB, Dinsmore RE, et al. A definition of advanced types of atherosclerotic lesions and a histological classification of atherosclerosis: $A$ report from the Committee on Vascular Lesions of the council on arteriosclerosis, American heart association. Circulation. 1995;92(5):13551374.

22. Paterson JC. Capillary rupture with intimal hemorrhage as a cause of pulmonary thrombosis. Am Heart J. 1939;18(4):451-457.

23. Michel JB, Virmani R, Arbustini E, Pasterkamp G. Intraplaque haemorrhages as the trigger of plaque vulnerability. Eur Heart J. 2011;32(16):1977-1985.

24. Jin $\mathrm{H}$, Goossens $\mathrm{P}$, Juhasz $\mathrm{P}$, et al. Integrative multiomics analysis of human atherosclerosis reveals a serum response factor-driven network associated with intraplaque hemorrhage. Clin Transl Med. 2021;11(6):11.

25. Gombozhapova A, Rogovskaya Y, Shurupov V, et al. Macrophage activation and polarization in post-infarction cardiac remodeling. J Biomed Sci. 2017;24(1):1-11.

26. Mouton AJ, DeLeon-Pennell KY, Rivera Gonzalez OJ, et al. Mapping macrophage polarization over the myocardial infarction time continuum. Basic Res Cardiol. 2018;113(4).

27. Van Der Laan AM, Ter Horst EN, Delewi R, et al. Monocyte subset accumulation in the human heart following acute myocardial infarction and the role of the spleen as monocyte reservoir. Eur Heart J. 2014;35(6):376385.

28. Rienks M, Carai P, Bitsch N, et al. Sema3A promotes the resolution of cardiac inflammation after myocardial infarction. Basic Res Cardiol. 2017;112(4).

29. Xue J, Schmidt SV, Sander J, et al. Transcriptome-Based Network Analysis Reveals a Spectrum Model of Human Macrophage Activation. Immunity. 2014;40(2):274-288.

30. Stoeckius M, Hafemeister C, Stephenson W, et al. Simultaneous epitope and transcriptome measurement in single cells. Nat Methods. 2017;14(9):865868.

31. Perisic L, Aldi S, Sun Y, et al. Gene expression signatures, pathways and networks in carotid atherosclerosis. J Intern Med. 2016;279(3):293-308.

32. Matic LP, Jesus Iglesias $M$, Vesterlund $M$, et al. Novel Multiomics Profiling of Human Carotid Atherosclerotic Plaques and Plasma Reveals Biliverdin Reductase B as a Marker of Intraplaque Hemorrhage. JACC Basic to Transl Sci. 2018;3(4):464-480.

33. Verhoeven BAN, Velema E, Schoneveld AH, et al. Athero-express: Differential atherosclerotic plaque expression of mRNA and protein in 
relation to cardiovascular events and patient characteristics. Rationale and design. Eur J Epidemiol. 2004;19(12):1127-1133.

34. Hellings WE, Peeters W, Moll FL, et al. Composition of carotid atherosclerotic plaque is associated with cardiovascular outcome: A prognostic study. Circulation. 2010;121(17):1941-1950.

35. Franzén $\mathrm{O}$, Ermel $\mathrm{R}$, Cohain $\mathrm{A}$, et al. Cardiometabolic risk loci share downstream cis- and trans-gene regulation across tissues and diseases. Science (80- ). 2016;353(6301):827-830.

36. Langley SR, Dwyer J, Drozdov I, Yin X, Mayr M. Proteomics: From single molecules to biological pathways. Cardiovasc Res. 2013;97(4):612-622.

37. Tomas L, Edsfeldt A, Mollet IG, et al. Altered metabolism distinguishes highrisk from stable carotid atherosclerotic plaques. Eur Heart $J$. 2018;39(24):2301-2310.

38. Williams JW, Winkels H, Durant CP, Zaitsev K, Ghosheh Y, Ley K. Single Cell RNA Sequencing in Atherosclerosis Research. Circ Res. Published online April 24, 2020:1112-1126.

39. Brbić $M$, Zitnik $M$, Wang $S$, et al. MARS: discovering novel cell types across heterogeneous single-cell experiments. Nat Methods. Published online 2020.

40. Wirka RC, Wagh D, Paik DT, et al. Atheroprotective roles of smooth muscle cell phenotypic modulation and the TCF21 disease gene as revealed by single-cell analysis. Nat Med. 2019;25(8):1280-1289.

41. Fernandez DM, Rahman AH, Fernandez NF, et al. Single-cell immune landscape of human atherosclerotic plaques. Nat Med. 2019;25(10):15761588.

42. Depuydt MAC, Prange KHM, Slenders L, et al. Microanatomy of the Human Atherosclerotic Plaque by Single-Cell Transcriptomics. Circ Res. 2020;127:1437-1455.

43. Zernecke A, Winkels $\mathrm{H}$, Cochain $\mathrm{C}$, et al. Meta-analysis of leukocyte diversity in atherosclerotic mouse aortas. Circ Res. 2020;127(3):402-426.

44. Subramanian A, Tamayo P, Mootha VK, et al. Gene set enrichment analysis: A knowledge-based approach for interpreting genome-wide expression profiles. Proc Natl Acad Sci U S A. 2005;102(43):15545-15550.

45. Consortium TGO, Carbon S, Douglass E, et al. The Gene Ontology resource: enriching a GOld mine. Nucleic Acids Res. 2021;49(D1):D325-D334.

46. Kanehisa M, Araki M, Goto S, et al. KEGG for linking genomes to life and the environment. Nucleic Acids Res. 2008;36(SUPPL. 1):D480-D484.

47. Fabregat $A$, Jupe $S$, Matthews $L$, et al. The Reactome Pathway Knowledgebase. Nucleic Acids Res. 2018;46(D1):D649-D655.

48. Martens $M$, Ammar A, Riutta $A$, et al. WikiPathways: connecting communities. Nucleic Acids Res. 2021;49(D1):D613-D621.

49. Rapaport F, Khanin R, Liang Y, et al. Comprehensive evaluation of differential 
gene expression analysis methods for RNA-seq data. Genome Biol. 2013;14(9):R95.

50. Peng $\mathrm{H}$, Long $\mathrm{F}$, Ding $\mathrm{C}$. Feature selection based on mutual information: Criteria of Max-Dependency, Max-Relevance, and Min-Redundancy. IEEE Trans Pattern Anal Mach Intell. 2005;27(8):1226-1238.

51. Larrañaga $P$, Calvo $B$, Santana $R$, et al. Machine learning in bioinformatics. Brief Bioinform. 2006;7(1):86-112.

52. Sidey-Gibbons JAM, Sidey-Gibbons CJ. Machine learning in medicine: a practical introduction. BMC Med Res Methodol. 2019;19(1):64.

53. Szegedy C, Liu W, Jia Y, et al. Going deeper with convolutions. In: Proceedings of the IEEE Computer Society Conference on Computer Vision and Pattern Recognition. Vol 07-12-June-2015. IEEE Computer Society; 2015:1-9.

54. Hastie T, Tibshirani R, Friedman J. The Elements of Statistical Learning. Springer New York; 2009.

55. Saeys $Y$, Inza I, Larranaga $P$. A review of feature selection techniques in bioinformatics. Bioinformatics. 2007;23(19):2507-2517.

56. Guyon I, Elisseeff A. An Introduction to Variable and Feature Selection. Vol 3.; 2003.

57. Butler A, Hoffman P, Smibert P, Papalexi E, Satija R. Integrating single-cell transcriptomic data across different conditions, technologies, and species. Nat Biotechnol. 2018;36(5):411-420.

58. Van Der Maaten L, Hinton G. Visualizing Data Using T-SNE. Vol 9.; 2008.

59. Mcinnes L, Healy J, Melville J. UMAP: Uniform Manifold Approximation and Projection for Dimension Reduction.; 2018.

60. Stuart T, Butler A, Hoffman P, et al. Comprehensive Integration of Single-Cell Data. Cell. 2019;177(7):1888-1902.e21.

61. Matsunaga $\mathrm{H}$, Ito K, Akiyama M, et al. Transethnic Meta-Analysis of Genome-Wide Association Studies Identifies Three New Loci and Characterizes Population-Specific Differences for Coronary Artery Disease. Circ Genomic Precis Med. 2020;13(3):2670.

62. Barabási A-L, Gulbahce N, Loscalzo J. Network medicine: a network-based approach to human disease. Nat Rev Genet. 2011;12(1):56-68.

63. Albert $\mathrm{R}$, Jeong $\mathrm{H}$, Barabási AL. Error and attack tolerance of complex networks. Nature. 2000;406(6794):378-382.

64. Jeong $\mathrm{H}$, Mason SP, Barabási AL, Oltvai ZN. Lethality and centrality in protein networks. Nature. 2001;411(6833):41-42.

65. Csardi G, Nepusz T. The igraph software package for complex network. InterJournal, complex Syst. 2006;1695(5):1-9.

66. Zhang B, Horvath S. A general framework for weighted gene co-expression network analysis. Stat Appl Genet Mol Biol. 2005;4(1). 
67. Mostafavi S, Ray D, Warde-Farley D, Grouios C, Morris Q. GeneMANIA: A real-time multiple association network integration algorithm for predicting gene function. Genome Biol. 2008;9(SUPPL. 1):1-15.

68. Langfelder P, Horvath S. WGCNA: An R package for weighted correlation network analysis. BMC Bioinformatics. 2008;9(1):1-13.

69. Gargalovic PS, Imura M, Zhang B, et al. Identification of inflammatory gene modules based on variations of human endothelial cell responses to oxidized lipids. Proc Natl Acad Sci U S A. 2006;103(34):12741-12746.

70. Pei G, Chen L, Zhang W. WGCNA Application to Proteomic and Metabolomic Data Analysis. In: Methods in Enzymology. Vol 585. Academic Press Inc.; 2017:135-158.

71. Janky R, Verfaillie A, Imrichová H, et al. iRegulon: From a Gene List to a Gene Regulatory Network Using Large Motif and Track Collections. PLoS Comput Biol. 2014;10(7):e1003731.

72. Davidson E, Levine M. Gene regulatory networks. Proc Natl Acad Sci U S A. 2005;102(14):4935.

73. Park PJ. ChIP-seq: Advantages and challenges of a maturing technology. Nat Rev Genet. 2009;10(10):669-680.

74. Szklarczyk D, Gable AL, Lyon D, et al. STRING v11: protein-protein association networks with increased coverage, supporting functional discovery in genome-wide experimental datasets. Nucleic Acids Res. 2018;47:607-613.

75. Patil KR, Nielsen J. Uncovering transcriptional regulation of metabolism by using metabolic network topology. Proc Natl Acad Sci U $S$ A. 2005;102(8):2685-2689. 




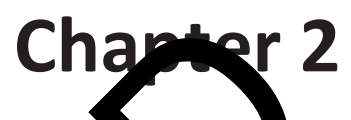

\section{Identification of a PRDM1-driven gengye rory to} regulate $T$ cell activity in high-risk up an praque

Han Jin, Emiel van der Vorst, Sanne L. Maas, Ch hg Lu, Ja Nagenborg, Marco Manca, Joël M.H. Karel, Rachel Cavill, Olivia cring, Junel. J. Sikkink, Barend M.E. Mees, Mat J.A.P. Daemen, Evgueni Sr rnov, th C. Sluimer, Erik A.L. Biessen

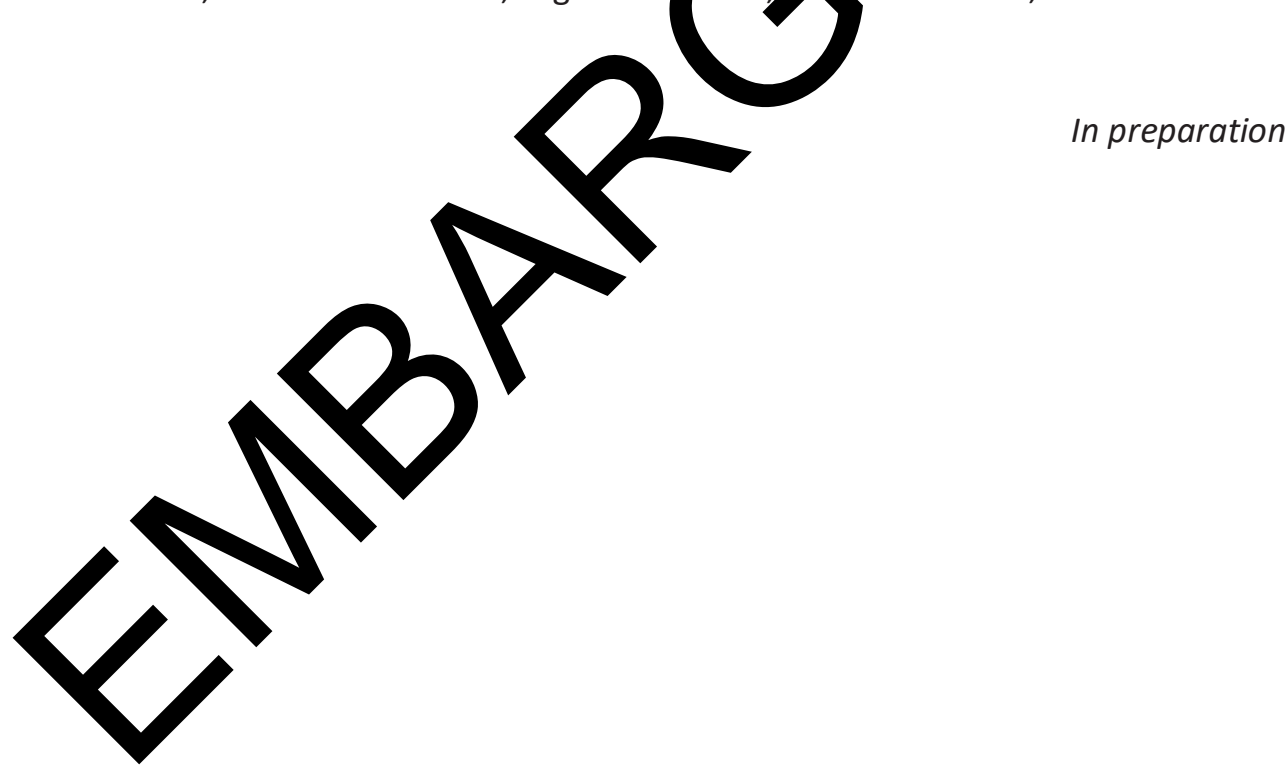




\section{Chapter 3}

\section{Integrative multi-omics analysis of human atherosclerosis reveals a serum response factor- driven network associated with intraplaque haemorrhage}

Han Jin, Pieter Goossens, Peter Juhasz, Wouter Eijgelaar, Marco Manca, Joël Karel, Evgueni Smirnov, Cornelis J.J.M. Sikkink, Barend M.E. Mees, Olivia Waring, Kim van Kuijk, Gregorio E. Fazzi, Marion J.J. Gijbels, Martina Kutmon, Chris T.A. Evelo, Ulf Hedin, Mat J.A.P. Daemen, Judith C. Sluimer, Ljubica Matic, Erik A.L. Biessen 



\section{ABSTRACT}

While single-omics analyses on human atherosclerotic plaque have been very useful to map stage- or disease-related differences in expression, they only partly capture the array of changes in this tissue and suffer from scale-intrinsic limitations. In order to better identify processes associated with intraplaque haemorrhage and plaque instability, we therefore combined multiple omics into an integrated model.

In this study, we compared protein and gene makeup of low- vs high-risk atherosclerotic lesion segments from carotid endarterectomy patients, as judged from the absence or presence of intraplaque haemorrhage, respectively. Transcriptomic, proteomic and peptidomic data of this plaque cohort were aggregated and analysed by DIABLO, an integrative multivariate classification and feature selection method.

We identified a protein-gene associated multi-omics model able to segregate stable, non-haemorrhaged from vulnerable, haemorrhaged lesions at high predictive performance (AUC > 0.95). The dominant component of this model correlated with $\alpha S M A^{-P D G F R} \alpha^{+}$fibroblast-like cell content $(\mathrm{p}=2.4 \mathrm{E}-05)$ and $\mathrm{Arg}^{+}$macrophage content $(p=2.2 \mathrm{E}-04)$ and was driven by serum response factor (SRF), possibly in a megakaryoblastic leukemia-1/2 (MKL1/2) dependent manner. Gene set overrepresentation analysis on the selected key features of this model pointed to a clear cardiovascular disease signature, with overrepresentation of extracellular matrix synthesis and organization, focal adhesion and cholesterol metabolism terms, suggestive of the model's relevance for the plaque vulnerability. Finally, we were able to corroborate the predictive power of the selected features in several independent mRNA and proteomic plaque cohorts.

In conclusion, our integrative omics study has identified an intraplaque haemorrhage-associated cardiovascular signature provides excellent stratification of low- from high-risk carotid artery plaques in several independent cohorts. Further study revealed suppression of an SRF-regulated disease network, controlling lesion stability, in vulnerable plaque, which can serve as a scaffold for the design of targeted intervention in plaque destabilization. 


\section{INTRODUCTION}

Atherosclerosis and associated cardiovascular diseases remain one of the leading causes of death worldwide ${ }^{1}$. In the clinically relevant stage, atherosclerotic lesions may transition from a stable, low-risk to an unstable phenotype, at high risk of causing acute ischemic events, such as myocardial infarction and ischemic stroke ${ }^{2}$. Histopathology and experimental studies and more recently, genome-wide association studies (GWAS) and extensive omics searches have considerably enhanced our understanding of this disease's pathogenesis ${ }^{3}$.

So far, the latter studies have largely relied on global profiling of mRNA expression or protein abundance in plaque tissue and were primarily designed to dissect differential expression, leading amongst others to the identification of plaque contained biomarkers correlating with future cardiovascular events (e.g. osteopontin) ${ }^{4-6}$. For instance, based on mRNA expression data from the Biobank of Karolinska Endarterectomies (BiKE) cohort ${ }^{7}$, Diez et al. were able to identify novel gene networks operating in the atherosclerotic plaque ${ }^{8}$.

A limitation of such global approaches is that they often fail to reveal detailed information about a particular cellular component in plaques. Laser-capture microscopy ${ }^{9,10}$ and single-cell RNA sequencing-based analysis of atherosclerotic plaque macrophages ${ }^{11-13}$ bypassed this pitfall. These studies allowed assessment and deconvolution, respectively, of the expression profiles of relevant plaque contained subsets, and for the latter confirmed the strong proinflammatory nature of non-foamy plaque macrophages ${ }^{13}$.

However, for in-depth dissection of critical processes in plaque, mere transcriptional profiling may fall short of incorporating net biosynthesis and protein activity, whereas proteomics profiling alone will bias towards most abundant, rather than most relevant proteins, implying that both scales have serious shortcomings ${ }^{14}$. Moreover, protein and mRNA profiles are notorious for their rather poor mutual correlation ${ }^{15,16}$, complicating the translation of genomics findings to clinical practice. Therefore, it is important to interrogate plaques by multiple omics and to evaluate the integrated model for biological and/or clinical implications ${ }^{17}$, To this end, significant efforts have already been made, and a few recent studies have illustrated the potential of such approaches ${ }^{18-20}$, Applying differential expression and intersect analyses ${ }^{18}$ and/or linking omics with genetics data ${ }^{19,20}$, these studies largely ignored mutual interaction of gene and/or proteins, and, for the former, did not truly integrate the data scales in a multivariate manner ${ }^{21}$.

Therefore, in the current study we set out to integrate the protein, peptide and mRNA makeup of carotid artery lesions from the Maastricht Human Plaque Study (MaasHPS) $^{22}$ of symptomatic carotid endarterectomy (CEA) patients. We 
interrogated a total of 42 carotid artery lesions, based on the presence/absence of intraplaque haemorrhage (IPH), a hallmark of plaque stability and event risk ${ }^{23,24}$. By integrative machine learning ${ }^{25}$ we were able to identify a composite disease stage classifier, the performance of which was benchmarked against single-omics derived classifiers using only mRNA expression or protein/peptide abundance and validated in several independent plaque datasets. 


\section{METHODS}

\section{Sample collection and classification}

Atherosclerotic plaque samples obtained during carotid endarterectomy from 24 symptomatic patients were collected. All patient materials were collected in the Maastricht Pathology Tissue Collection in line with the Dutch Code for Proper Secondary Use of Human Tissue (https://www.federa.org) and the local Medical Ethical Committee (protocol number 16-4-181). This study conforms to the Declaration of Helsinki, all participants have given informed written consent prior to the inclusion. The endarterectomy specimens were cut into parallel, transverse segments of $5 \mathrm{~mm}$ thickness. Each alternating segment was snap-frozen in liquid nitrogen and stored at $-80^{\circ} \mathrm{C}$, their flanking segments fixed for 24 hours in formalin, decalcified for 4 hours before processing and embedding in paraffin for histological evaluation.

Haematoxylin-Eosin (H\&E) stained plaque tissue was macroscopically pre-classified for plaque stage before omics experiments. Segments were stratified into non-IPH and IPH groups according to the absence or presence of IPH. For each CEA specimen and patient, two samples (one non-IPH and one IPH) were collected for omics experiments ( $n=24$ at the start for each). However, one IPH and four non-IPH samples did not pass the QC test and were excluded from analysis (see the following sections). Simultaneously with the microarray performance for the omics experiments, but prior to bioinformatic analysis, tissue was sectioned further for additional staining and computer-aided quantitative measurement of plaque IPH. Based on this in-depth reinspection, the three experienced pathologists (MJAPD, $J C S, M J J G$ ) agreed to remove one ambiguous "non-IPH" sample, showing small but surface-detached luminal fibrin clot, as this could represent either a surgery artefact or bona fide IPH. In three allegedly "non-IPH" samples, quantitative morphometry detected minor IPH $(0.43 \%, 0.40 \%, 0.33 \%$, respectively), which was overlooked in the pre-classification. These three were recategorized as IPH after inspection by the pathologists. Due to the recategorization of samples from the non-IPH to the IPH group, three pairs of the total 26 samples of the IPH group were from three patients, respectively. To explore the potential confounder effects of this adjustment, we performed hierarchical clustering of the samples based on the plaque traits (measured as described in the following section). This analysis convincingly showed that there is no patient-specific heterogeneity among the samples, as well as that plaque phenotype is dominant over sample origin (data not shown). The final cohort for this study therefore included transcriptomics, proteomics and peptidomics for $16 \mathrm{non}-\mathrm{IPH}$ and $26 \mathrm{IPH}$ plaques (see Figure 1A-B for flow scheme of cohort build-up). For detailed information on the patient cohort definition see Table S1.

Page | 74 


\section{Morphometry and immunohistochemistry}

All H\&E slides were photographed at a $12.5 \times$ magnification and examined digitally using Leica Q500MC software. Total tissue, media, cap, necrotic core, haemorrhage and luminal thrombus area were measured on H\&E. Plaque size was calculated by subtracting the medial and luminal thrombus area from that of the total carotid tissue. Necrotic core and haemorrhage area were quantified relative to the plaque size. Cap thickness was measured at 3-15 positions throughout the entire cap region, at regular intervals. In addition, we measured the relative area of the following histological features: $\mathrm{CD}_{3} 1^{+}$endothelial cell content (\% of total plaque area), $\mathrm{CD}^{+} \mathrm{T}$-cell content (\% of total plaque area), $\mathrm{CD}^{+} 8^{+}$macrophage content (\% of total plaque area), iNOS ${ }^{+}$(M1) macrophage content (\% of $\mathrm{CD}^{+} 8^{+}$macrophage area), $\operatorname{Arg}^{+}(\mathrm{M} 2)$ macrophage content (\% of $\mathrm{CD}^{+} 8^{+}$macrophage area), collagen content ( $\%$ Sirius red of total plaque area), $\alpha \mathrm{SMA}^{+}$smooth muscle cell (SMC) content ( $\%$ of total plaque area), $\alpha$ SMA-PDGFR $\alpha^{+}$fibroblast-like cell content ( $\%$ of total plaque area), and calcification (\% Alizarin red of total plaque area). These histological features are denoted as plaque traits, their distribution and statistical differences between paired non-IPH and IPH plaques are shown in Figure S1. Moreover, a correlation heatmap was created to explore the relationships between the plaque traits (Figure S2).

For the MFAP4 immunohistochemistry for validation purposes, four- $\mu m$ thick sections were cut from 35 plaques (13 non-IPH, 22 IPH plaques) left available from the total 42 plaques. Deparaffinization and rehydration were performed using xylene and graded ethanol. Endogenous peroxidase was blocked with $0.3 \% \mathrm{H}_{2} \mathrm{O}_{2}$ for 15 minutes followed by heat-induced antigen retrieval in a microwave for 10 minutes in a low pH target retrieval solution (K8005, DAKO, Agilent Technologies). After cooling down for 20 minutes, sections were incubated overnight at $4{ }^{\circ} \mathrm{C}$ with polyclonal rabbit anti-human MFAP4 antibody (1:1000, NBP2-30439: Novus Biologicals). After washing in TBS, sections were incubated for 1 hour at room temperature in Brightvision poly-HRP anti-rabbit IgG (ImmunoLogic, VWR KDPVR55 HRP). After washing in TBS, staining was developed using the chromogen 3,3'diaminobenzide (DAB, DAKO K3468) for 5 minutes and sections were counterstained with Mayer's Haematoxylin (VWR International B.V., Amsterdam, The Netherlands), dehydrated, mounted and cover slipped. Sections were digitized using the Pannoramic 1000 scanner (3DHistech Ltd.) at 20x magnification. Morphometric analysis was done using the free software package QuPath (v0.2.3) to measure $\%$ area positivity / total area.

\section{Sample preparation for transcriptomics and proteomics/peptidomics}

Snap-frozen omics segments were pulverized and 5 to $20 \mathrm{mg}$ of material was subjected to transcriptomic, proteomic, and peptidomic analysis. 
RNA was isolated by Guanidium Thiocyanate extraction, followed by further purification using the Nucleospin RNA II kit (Macherey-Nagel GmbH \& Co. KG). RNA concentration was measured using a Nanodrop ND-1000 spectrophotometer (Nanodrop Technologies, Wilmington, DE, U.S.A). RNA quality and integrity were determined on the Agilent 2100 Bioanalyzer (Agilent Technologies, Inc., Santa Clara, CA, U.S.A.). Pure RNA samples that had an RNA Integrity Number (RIN) greater than 6.0 and a sample purity A260/280 ratio greater than 1.8 were taken for transcriptomic analysis (45 out of 48 samples). One analysis for a non-IPH plaque had failed, therefore in total four unqualified non-IPH samples were excluded from the microarray experiment.

Proteomic analysis was carried out by utilizing the 8-plex iTRAQ reagent for relative quantification. Reference sample used for relative quantification was created by pooling the redundant specimens derived from the equal portions of the samples for omics experiments. Pulverized tissue samples $(5-20 \mathrm{mg})$ were homogenized in a Covaris E100 ultrasonic homogenizer using $400 \mu \mathrm{L}$ lysis buffer of $6 \mathrm{M}$ guanidium hydrochloride, 1\% Triton-114, $50 \mathrm{mM}$ triethylammonium bicarbonate (TEAB), 50 $\mathrm{mM}$ dithiothreitol (DTT), and protease inhibitor tablets (Roche, $1 / 4$ tablet in $10 \mathrm{~mL}$ buffer). The homogenate was centrifuged for $15 \mathrm{~min}$ at 4,000x $\mathrm{g}$ to remove insoluble debris. DTT content of the homogenate was raised to $100 \mathrm{mM}$ and protein reduction was completed through incubation at $70{ }^{\circ} \mathrm{C}$ for an hour. Alkylation was completed by adding $0.5 \mathrm{M}$ iodoacetamide and followed by a 30-min incubation at room temperature. The excess alkylating reagent was quenched by the addition of $12 \mu \mathrm{L}$ of $1 \mathrm{M}$ DTT. The protein content of the homogenate was recovered on a Poros R1 column using a Vision Chromatography Station (Applied Biosystems). Proteins were eluted with $70 \%$ isopropanol/30\% formic acid solvent mixture and dried down in a Speedvac.

\section{Omics data collection}

Biotinylated cRNA was prepared using the Illumina TotalPrep RNA Amplification Kit (Ambion, Inc., Austin, TX, U.S.A.) according to the manufacturer's specifications starting with $100 \mathrm{ng}$ total RNA. Per sample $750 \mathrm{ng}$ of cRNA was used for hybridization. Hybridization and washing were performed according to the Illumina standard assay procedure. Scanning was performed on the Illumina BeadStation 500 (Illumina, Inc., San Diego, CA, U.S.A.), while image analysis and extraction of raw expression data were done using Illumina Beadstudio v3 Gene Expression software with default settings (no background subtraction and no normalization). Transcripts were measured by Illumina HumanRef-8 v2.0 expression BeadChip ${ }^{\circledR}$.

Proteins were resuspended in $2 M$ freshly prepared urea, $1 M$ TEAB, $1 \%$ n-octylglucoside buffer and digested with trypsin added at 1:20 w/w ratio to the sample, for 4 hours at $37^{\circ} \mathrm{C}$. Digestion was stopped by increasing the temperature to $95^{\circ} \mathrm{C}$ 
for 5 minutes. Digested samples were labelled by the 8-plex iTRAQ reagents following the manufacturer's protocols (Applied Biosystems) using a sample digest quantity that represents approximately $40 \mu \mathrm{g}$ protein content. Samples were labelled with the reagents yielding the $\mathrm{m} / \mathrm{z} 114,115,116,118,119,121$ reporter fragments in the MS/MS scans. Reference samples were labelled with the 113 and 117 reagents. ITRAQ labelled reactions were quenched by the addition of $1 \mathrm{M}$ ammonium bicarbonate.

Eight samples (three non-IPH, three IPH, and two replicates for QC and reference) constituting an ITRAQ mix were combined, desalted and fractionated by strong cation exchange (SCX) chromatography using an Agilent 1200 instrument. Eight SCX fractions were injected for HPLC and resolved over a 90 -min gradient of $5 \%$ solvent B (10\% H2O/90\% ACN/0.1\% TFA) to 38\% B (solvent A: 95\% H2O/5\% ACN/0.1\% TFA). The elution volume was collected onto a plate for mass spectrometric analysis by matrix-assisted laser desorption ionization (MALDI) as 10-sec intervals, using 10 $\mathrm{mg} / \mathrm{mL}$ of alpha-cyano-4-hydroxycinnamic acid in $50-50 \%$ acetonitrile-water as the matrix. Each HPLC run was represented as a 500-spot array on the MALDI plate. These plates were analysed on an AB4800 mass spectrometer (MDS/SCIEX, Concord, ON, Canada).

Peptide quantification was carried out by calculating the average ion intensity ratios relative to the $\mathrm{m} / \mathrm{z} 113$ and 117 peaks. Protein ratios were determined as the medians of all peptide ratios matching to the same protein. Peptide sequences were identified from MS/MS fragmentation spectra using the Mascot search engine (Matrix Science, UK) and the SwissProt database. The whole proteomic/peptidomic analysis was carried out following the same protocols in a previous plasma proteomics study for cardiovascular disease ${ }^{26}$. Experimental details and parameters used in this analysis can be found from the paper give above. Once all the study samples were analysed, the complete peptide set was re-mapped to a minimum protein set, whereafter proteins and peptides identified were mapped to genes or gene families.

The corresponding microarray profile of one IPH sample with failed MS measurement (due to technical reasons) was removed. The final MaasHPS cohort therefore included transcriptomics for 16 non-IPH and $26 \mathrm{IPH}$ plaques with successful proteomics and peptidomics profiling from the same samples.

\section{Data preprocessing}

A total of 22,184 human transcripts and variants as defined by the NCBI Reference Sequence (RefSeq) were analysed. Transcriptomic data were analysed using the $R$ Bioconductor lumi package (v2.38.0). First, we performed a variance stabilizing transformation, which is incorporated in the lumi package. Then, the robust spline 
normalization (RSN) algorithm in the lumi package was applied to normalize the data. As low-variance genes and noise expression will not only reduce the effectiveness of subsequent clustering and machine learning, but also slow down computations, we selected the top 10,000 most variable genes from a total pool of 17,759 unique detectable genes, for further analysis.

Measured values of the abundance of 1,330 proteins and 4,736 peptides were normalized using a procedure based on Vandesompele et $\mathrm{al}^{27}$. Intrinsic to the analysis methodology, datasets were showing a considerable rate of missing values ( $26.45 \%$ and $38.94 \%$, respectively). To reduce the noise and bias affected by features with sub- and peri-threshold abundance, we discarded all features with $\geq$ $50 \%$ missing values. For the remaining features, missing values were imputed by $\mathrm{k}$ nearest neighbours ( $k-N N)$ imputation $(k=7)$ in an unsupervised manner, as this proved superior to other methods in a pilot analysis ${ }^{28}$.

Finally, 10,000 genes, 943 proteins, and 2,637 peptides were detectable in the 42 samples ( 16 non-IPH vs 26 IPH) and were used for further analysis. Genes, proteins, and peptides were mapped based on the HUGO Gene Nomenclature Committee (HGNC) symbols.

\section{Single- and multi-omics data analysis}

For single-omics analysis of the transcriptomic, proteomic and peptidomic data, we deployed sparse Partial Least Squares -Discriminant Analysis (sPLS-DA, or single sPLS-DA) $)^{29,30}$, a multivariate methodology which allows sample classification and feature selection by projecting the data into a lower-dimensional space in a supervised manner. While applying this method on omics datasets, which typically have a high-dimensional feature space with limited sample size, this algorithm implements LASSO ${ }^{31}$ to limit the number of features used in the model so as to reduce the curse of dimensionality. To enable classification based on multi-block datasets, derived from transcriptomic, proteomic, and peptidomic analysis of plaques, we deployed integrative SPLS-DA using DIABLO provided in the mixOmics $\mathrm{R}$ package (http://mixomics.org/, v6.8.5) ${ }^{25}$, a powerful package shown to extract biologically relevant signatures from multiple omics data by maximizing the sum of the covariance between all pairs of latent components from each dataset and projecting the different omics data into a common space ${ }^{32}$. Compared with other multi-omics machine learning algorithms, this method has several advantages. First, it allowed full integration of multi-omics data by extracting common information from different omics layers for classification. Second, it enables model-embedded feature selection, which is a great help for identifying disease-specific novel biomarkers. Third, this method provides clear and interpretable visualization, facilitating downstream exploration and interpretation by biologists. The inherent characteristics $^{33}$ and the successful applications of sPLS-DA on several microarray Page $\mid 78$ 
data $^{34,35}$ suggest the suitability of using this method in our study.

\section{Parameter optimization}

SPLS-DA requires extensive optimization of both the number of components and the number of features for constructing each component. In brief, the number of testing components was set from 1 to 6 , as in most cases, the prediction performance deteriorates rapidly when the number of the components increase above 5. Then, the number of features to be tested for constructing the sPLS-DA component was configured. For single sPLS-DA on transcriptomics, proteomics and peptidomics, a range of features from 3 to 300 (from 3 to 30 in steps of 3, from 30 to 60 in steps of 6, from 60 to 150 in the step of 15, and from 150 to 300 in the step of 30) were set for each component. For integrative SPLS-DA, to achieve the comparable number of selected features, the range of testing features here for each component and each omics was set from 10 to 100 (from 10 to 50 in steps of 5, from 50 to 100 in steps of 10, in total 14 candidates per component per omics).

Specifically, for integrative sPLS-DA, a design matrix representing how close the datasets should be connected to each other needs to be configured. According to Singh's suggestion ${ }^{36}$, we configured the design matrix $C$ as the correlation between omics datasets as 0.1 , and the correlation between omics dataset and outcome dummy matrix as 1.

Optimal parameters can be derived in one step from the model with the best classification in cross-validation. A series of single and integrative sPLS-DA prediction models were obtained from each combination of these parameter settings, and their performances were tested under stratified 5-fold crossvalidation with 1,000 random repeats. Considering the unbalance of the sample phenotypes, to fairly reflect the classification performance of training models, in this tuning phase we used balanced error rate (BER), which takes the average of the errors on each class to evaluate the classification error. The optimal parameters can be concluded from Figure S3A (for integrative sPLS-DA, data not shown) and were listed in Table S2. The performance of the single and integrative sPLS-DA models with the given optimal parameters was evaluated by accuracy and AUC under stratified 5-fold cross-validation with 10,000 random repeats (Figure S3B).

\section{Feature selection}

Inspired by the method used in a previous multi-omics study ${ }^{32}$, to ensure that selected features had broader significance beyond the single best prediction model, we performed stratified sampling with replacement generating 10,000 incomplete copies of the full dataset; each copy covered $80 \%$ of the original data, and phenotype distribution was identical to that of the original dataset. We then ran single- and multi-omics analysis on each copy, selecting the overall highest-ranking 
features by aggregating the loading weight value (i.e., the importance to the model) of each feature from each copy to obtain the final feature subset. The optimal parameters (Table S2) were used to determine the number of the selected features.

To simplify the downstream analysis, we assembled gene, peptide and protein identifiers of selected features into a final feature set, unifying the identifiers into the corresponding gene symbol and excluding duplications. The final selections used for overrepresentation analysis, transcriptional regulatory analysis and validation entailed 291 and 283 gene features, for single and integrative sPLS-DA, respectively.

\section{Bioinformatic analyses}

Differential gene expression analysis: We used the function ImFit() provided in the limma R package (v3.42.2) for differential expression analysis on preprocessed transcriptomics between IPH and non-IPH group. Positive log2 fold change (Log2FC) values indicate genes have higher expression in IPH group, vice versa.

Gene Set Overrepresentation Analysis (GSOA): We performed GSOA using the R packages clusterProfiler ${ }^{37}$ (v3.12.0) and ReactomePA ${ }^{38}$ (v1.28.0) identifying biological functions of the selected gene sets. Three categories of GSOA datasets were queried: Gene Ontology (Biological Process GOBP, Molecular Function GOMF, and Cellular Component GOCC), Reactome Pathway Database (REACTOME), and Kyoto Encyclopedia of Genes and Genomes (KEGG). All genes covered by this Illumina microarray platform and all detectable proteins/peptides in the MS experiments were used as the background. Benjamini-Hochberg adjusted $p$-values were calculated as a cut-off to avoid presenting the false discovery of the significant terms.

Transcriptional Regulation Analysis: iRegulon ${ }^{39}$ (v1.3), a Cytoscape plugin, was used to map transcription factors (TFs) driving a gene network. The selected features by integrative SPLS-DA were set as input in iRegulon for transcription factor discovery. By setting the default parameters, we have a broad search space including 9,713 position weight matrices (PWMs) and 1,120 ChIP-seq tracks. The putative regulatory region was set to $20 \mathrm{~kb}$ centred around transcription start sites (TSS). Top 5 TFs were ranked by normalized enrichment score and visualized as a transcriptional regulatory network.

\section{Validation cohorts and approach}

The predictive power of genes from the multi-omics signature was validated in the following independent human carotid endarterectomy cohorts on microarray gene expression: GSE28829 ${ }^{40}\left(n=29 ; 13\right.$ early and 16 advanced plaques) and GSE43292 ${ }^{4}$ ( $n=64 ; 32$ plaque-free artery segments and 32 atheromatous plaques).

Page $\mid 80$ 
The prediction performance of the gene lists obtained from integrative sPLS-DA component 1-4 (MULTI 1-4, $\mathrm{n}=283$ ) and single sPLS-DA component 1-2 (SINGLE, $\mathrm{n}$ $=291$, for peptidomics only component 1 ) were tested by logistic regression (solver = 'Ibfgs'), support vector machine (SVM, kernel = 'rbf', gamma = 'auto') and decision tree (default settings) with stratified 5 -folds cross-validation and 1,000 random repeats provided in the scikit-learn machine learning package (v0.24.1) in Python. As the integrative sPLS-DA component 1 (MULTI 1, $n=22$ ) was dominant in our model, we also compared the performance of the MULTI 1 signature with that of MULTI 1-4 signature from the complete 4-component model. Classification performance using full data (FULL) was evaluated as a baseline. Predictive performance was evaluated by two measures: Area Under the Curve of Receiver Operating Characteristic (AUC), and accuracy.

\section{BiKE verification}

BiKE cohort patient inclusion for CEA surgery and enrolment in the biobank, clinical data, sample processing and large-scale datasets have been described in detail previously ${ }^{7,18}$. In this study, the classification performance of MULTI 1 was also validated on transcriptomics ( $n=137 ; 127$ carotid plaques and 10 normal arteries) and proteomics ( $\mathrm{n}=36 ; 18$ carotid plaques and 18 adjacent, healthy, matched segments) from the BiKE cohort. Within the BiKE, two subcohorts were defined. For the first, we compared non-atherosclerotic tissues (normal; $n=10$ for transcriptomics, $\mathrm{n}=18$ for proteomics) versus atherosclerotic arteries (plaques; $\mathrm{n}$ $=127$ for transcriptomics, $n=18$ for proteomics), based on their morphological and histological characteristics (HIST). For the second, carotid plaque tissues were classified as asymptomatic ( $n=40$ for transcriptomics, $n=9$ for proteomics) vs symptomatic ( $n=87$ for transcriptomics, $n=9$ for proteomics) based on the patient's clinical presentation (CLIN). For both subcohorts, we tested the classification performance of the identified multi-omics signature MULTI 1 on transcriptomics and proteomics, respectively, using logistic regression with the same setting mentioned above for GSE28829 and GSE43292. Gene Set Enrichment Analysis (GSEA) was performed on the signature MULTI 1 against the genes and proteins from BiKE transcriptomics and proteomics, with the genes/proteins ranked by log 2 fold change based on the comparisons of HIST and CLIN.

\section{Statistical analysis}

Component-component and component-trait associations were measured by Pearson's Correlation Coefficients with p-values. Classification performances are presented as the mean \pm standard deviation (SD). Distribution of plaque traits and IHC-based MFAP4 quantification for paired plaques are presented as box and whisker plots with the first quartile, median, third quartile, and the largest or smallest values within 1.5 times the interquartile range above the third quartile or 
below the first quartile, respectively. Statistical significance between non-IPH and IPH groups was evaluated using two-tailed Wilcoxon rank-sum test (for nonnormally distributed data) or Student's t-test (for normally distributed data). For the MaasHPS data, paired statistical testing was performed. Shapiro-Wilk test was used for the normality test. Statistical significance between the classification performance was analysed by Student's t-test. Significance level is denoted by * $p$ value $<0.05, * * p$-value $<0.01,{ }^{* * *} p$-value $<0.001,{ }^{* * * *} p$-value $<0.0001$. Statistical analyses were performed in $\mathrm{R}$ (v3.6.3). 


\section{RESULTS}

In this study, we deeply phenotyped low- versus high-risk human carotid artery atherosclerotic lesion segments, as defined by the absence or presence of intraplaque haemorrhage (IPH), building an integrated gene/protein classification model for IPH. For this, we interrogated a carotid endarterectomy cohort (MaasHPS) by transcriptomic (microarray), proteomic and peptidomic analysis (both LCMALDI-MS/MS). Flanking sections were taken for detailed histological examination of cellular and acellular plaque composition and progression stage ${ }^{41}$ (Figure S1), resulting in 42 CEA samples (16 non-IPH vs 26 IPH, Table S1) from 24 patients (see Materials and methods section and Figure $1 A-B$ for cohort build-up scheme). After preprocessing, a total of 10,000 genes, 943 proteins, and 2,637 peptides were analysed according to the workflow described in Figure 1A. For clarity's sake, mRNAs/genes, proteins, and peptides from omics data are termed features and a set of features is termed signature. 


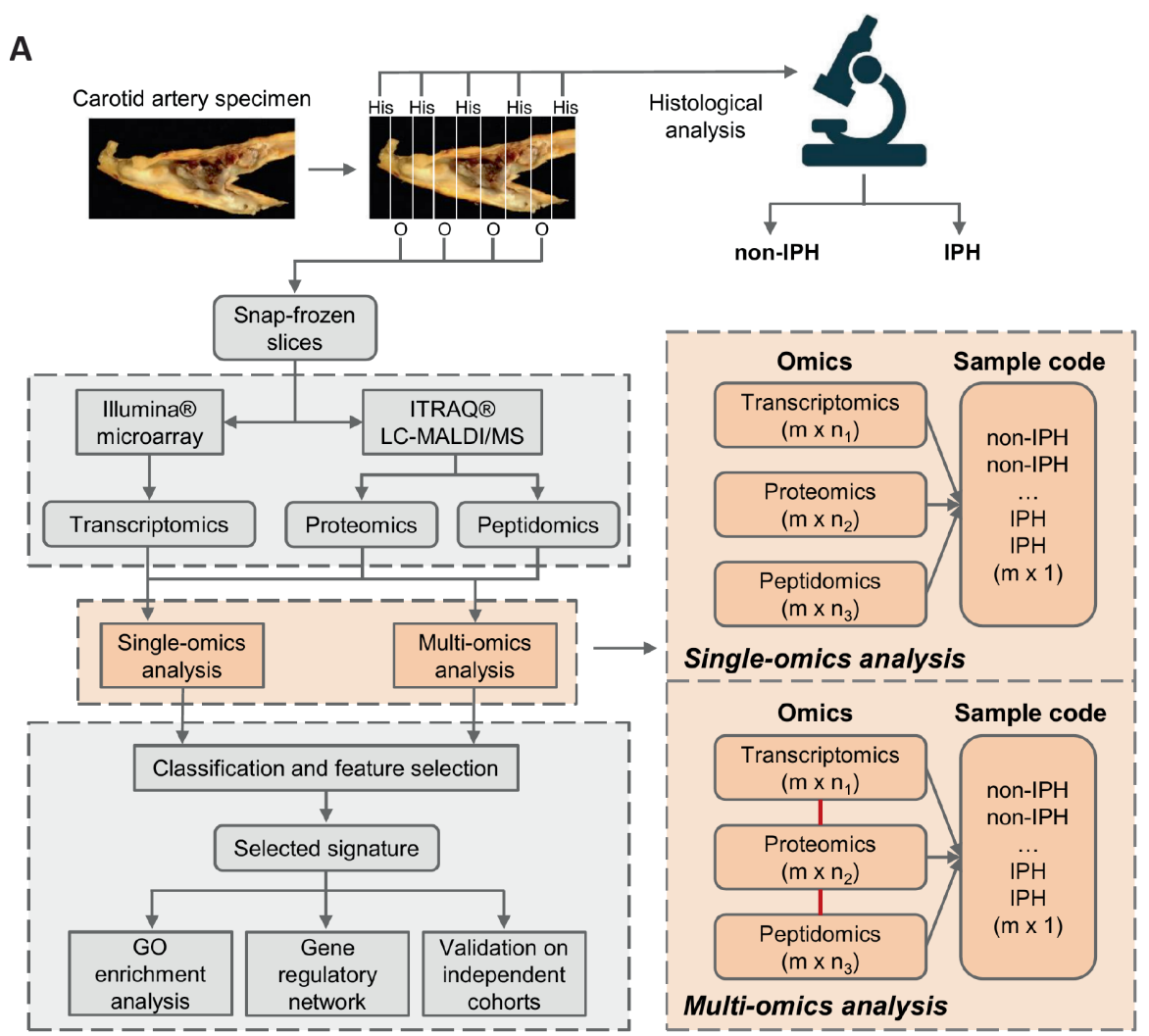

B

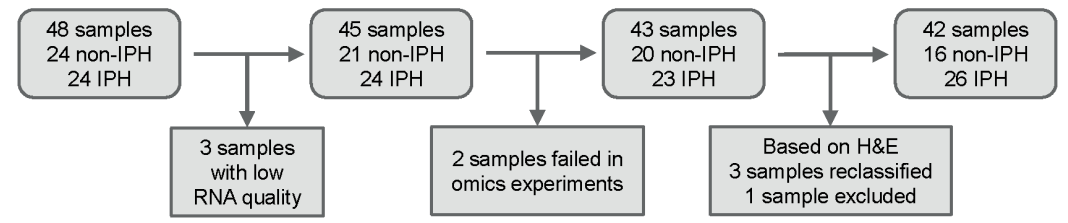

Figure 1. Workflow of carotid sample collection and data analysis

(A) The entire carotid endarterectomy specimen was cut in parallel $5 \mathrm{~mm}$ thick slices, snap-frozen in liquid nitrogen and stored until use. Every second slice was sectioned. After H\&E staining, sections were categorized using the criteria of Virmani, ${ }^{41}$ and were further classified histologically based on the presence/absence of IPH, resulting in 16 non-IPH and $26 \mathrm{IPH}$ plaques for omics analysis. Workflow for single- and multi-omics integrative SPLS-DA analysis are shown in the orange dashed square. The integrative SPLS-DA prediction model was obtained by connecting transcriptomics, proteomics and peptidomics as an entirety, m: number of samples, n1, n2, n3: number of features. (B) Flow scheme of the MaasHPS cohort Page | 84 
build-up and the criteria for sample exclusion. In total, 42 samples (16 non-IPH and $26 \mathrm{IPH}$ plaques) were included in this study.

\section{Single-omics sPLS-DA}

First, we built single sPLS-DA models based on the transcriptomic, proteomic, and peptidomic datasets, separately. The optimal parameters for model building can be found in Table S2 and Figure S3A. As is evident from Figure 2A, all three models were able to segregate IPH from non-IPH plaque at high predictive performance, with accuracies of approximately 0.9, and AUC's of > 0.95 (Figure S3B); in fact, nearly $90 \%$ of samples could be correctly classified by all three omics datasets separately. Next, we constructed heatmaps for the 3 omics layers, respectively, depicting the differential expression of signature members by IPH vs non-IPH plaque (Figure 2B and S4A). Component 1 gene, protein and peptide signatures showed strong differential expression between plaques with or without IPH. This is in support of the discriminative power of component 1 (Figure 2A), and suggests that the first component is the dominant factor in the prediction model.

Next, we mapped the distribution of the selected feature sets (Figure 2C), based on the Pearson's Correlation Coefficient between the latent components (i.e. component 1 and 2) and the selected features ${ }^{42}$. For both components selected features were distributed differently, centring towards the extreme ends of component 1 , and being more diffusely distributed for component 2 . Thus, unlike component 2, component 1 features showed pronounced mutual interaction, suggesting involvement in a coordinated biological process. Gene set overrepresentation analysis (GSOA) of the selected signatures for the first, decisive component revealed a clear overrepresentation of fibroblast and extracellular matrix-associated biological processes (Figure 2D). In contrast, but expected based on the diffuse distribution in Figure 2B-C, component 2 did not offer deeper insights into haemorrhage relevant processes. Of note, even for component 1 , the level of overrepresentation was rather low, precluding firm conclusions based on single sPLS-DA only. 


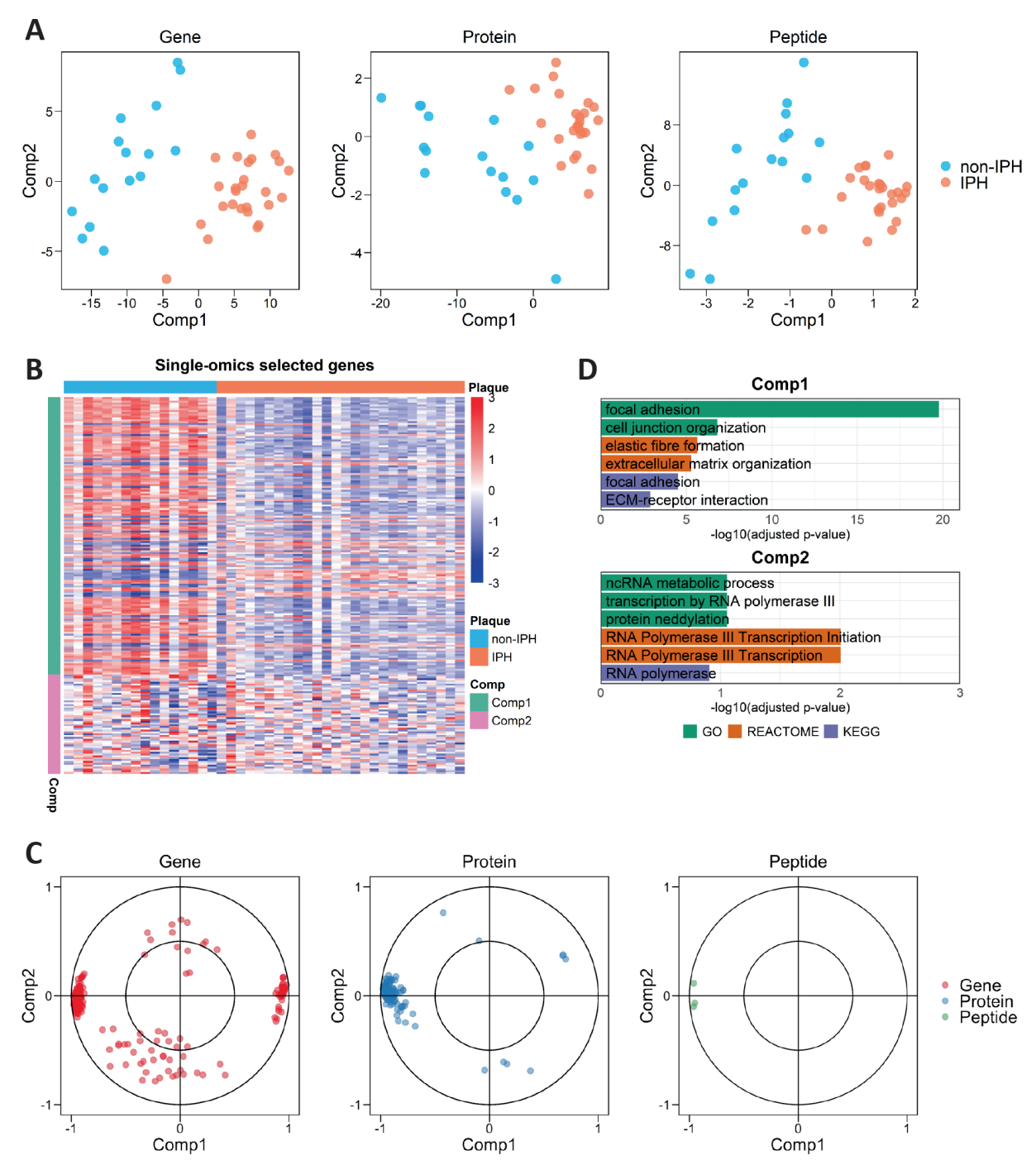

Figure 2. Single-omics SPLS-DA on transcriptomics, proteomics, and peptidomics (A) 2-component sample distribution (16 non-IPH and 26 IPH) for single-omics sPLSDA on plaque transcriptomics (gene), proteomics (protein), and peptidomics (peptide), respectively. For visualization purposes, two components were included in the plot for peptidomics. (B) Heatmap shows expression level of the selected genes (comp1, $n=150$; comp2, $n=54$ ) from transcriptomics. Expression values were z-normalized per row. (C) Selected features by single sPLS-DA on the three omics are plotted respectively ( $n=204$ for genes; $n=111$ for proteins; $n=3$ for peptides), according to their Pearson's Correlation Coefficient to the component 1 
and 2. (D) GSOA on standardized gene set of single sPLS-DA. For each component, six highly ranked overrepresented terms are selectively shown.

\section{Multi-omics sPLS-DA}

In order to establish a more comprehensive and robust model of intraplaque haemorrhage, we sought to analyse plaque transcriptomic, proteomic and peptidomic data in an integrated manner by integrative sPLS-DA using the optimized parameters in Table S2. The resulting prediction model provided excellent discrimination of non-IPH vs IPH plaque (Figure 3A), and performed at least equally good as all of the single-omics models, in terms of accuracy and AUC (Figure S3B). The features of the $1^{\text {st }}$, but not $2^{\text {nd }}-4^{\text {th }}$ components, showed overt differential expression between non-IPH and IPH plaque (Figure 3B and S4B), regardless of their origin (i.e., gene, protein, peptide). Furthermore, the selected proteins and peptides in Figure 3B showed considerable overlap (12 out of 20). Also, for each of the other three components, part of the selected proteins and peptides are overlapping, implying the contribution of the proteomics and peptidomics to the multi-omics model are to some extent similar but not identical. Crosscomponent correlation analysis confirmed the strong mutual correlation of component members from the three omics layers, whereas cross-interaction across the components was very weak (Figure $3 \mathrm{C}$ ), underpinning the high level of independence of the four components of our model.

To add biological meaning to the prediction model, we set out to identify cofunctional gene/protein/peptide clusters, correlating with each of the four components of the integrative model (Figure 3D). Several clusters were observed, suggesting a very strong mutual correlation between genes, proteins and peptides. This points to coordinate function and/or regulation of the cluster members. GSOA of the gene, protein and peptide members underlying the integrated classification model showed a clear cardiovascular signature (Figure 3E), with marked overrepresentation of extracellular matrix organization (component 1 and 3), lipid metabolism (component 2), and immune response terms (component 4). Interestingly, component 1 and 3 showed considerable functional overlap, despite their low interdependence. Of note, the first component of the multi-omics model was very comparable to that for the single-omics models, and showed a significant level of overlap (Figure 3F). To validate the GSOA findings, we correlated the four components with several important plaque traits, such as intraplaque haemorrhage, fibrosis and macrophage presence (Figure 4A). The dominant first component was highly correlated with plaque size (correlation $=0.74, p=1.8 \mathrm{E}-08$ ) and haemorrhaged plaque area (correlation $=0.48, p=1.4 \mathrm{E}-03$ ), as expected, as well as with $\alpha$ SMA-PDGFR $\alpha^{+}$fibroblast-like cells (correlation $=0.64, p=2.4 \mathrm{E}-05$ ), $\operatorname{Arg} 1^{+}$ healing macrophage phenotype (correlation $=-0.62, p=2.2 \mathrm{E}-04$ ), and collagen 
content of non-IPH plaque (correlation $=0.72, p=3.6 \mathrm{E}-03$ ), concordant with the GSOA findings (Figure 3E). Indeed, plaque size, haemorrhage area, collagen content and $\alpha S_{M A}{ }^{-P D G F R} \alpha^{+}$cell content were significantly correlated with each other (Figure S2), possibly explaining the correlations between component 1 and these plaque traits (Figure 4A).

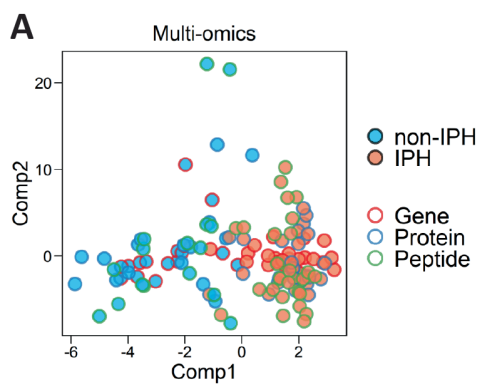

B

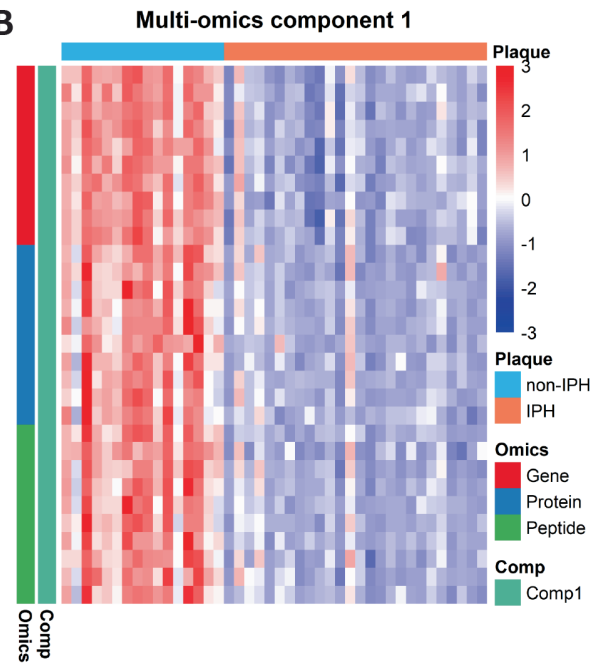

C Multi-omics component-component correlation

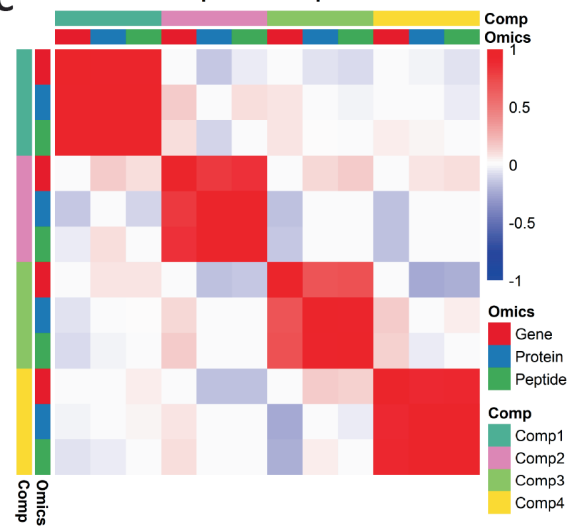

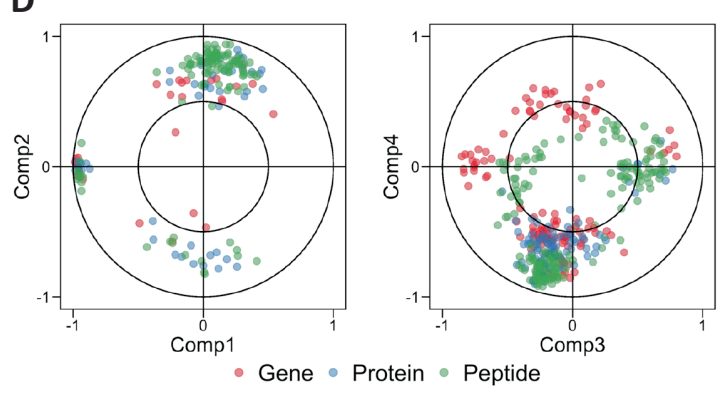

E
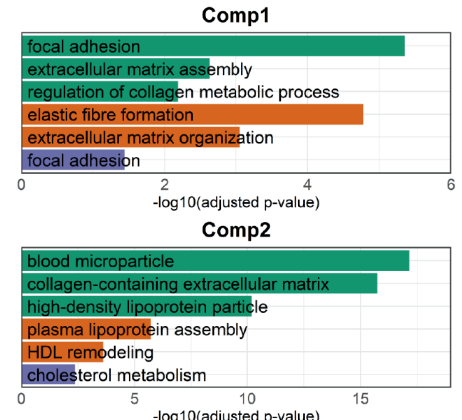

Comp3

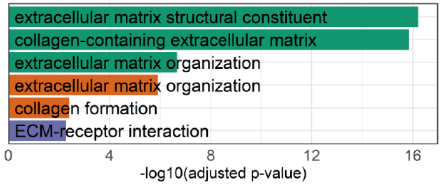

Comp4

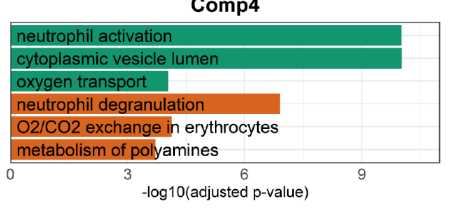

GO REACTOME — KEGG

$\mathbf{F}$

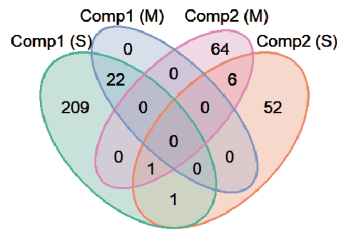


Figure 3. Multi-omics sPLS-DA on transcriptomics, proteomics, and peptidomics

(A) 2-component sample distribution for multi-omics SPLS-DA on plaque transcriptomics (gene), proteomics (protein), and peptidomics (peptide). Non-IPH and IPH plaques are denoted in skyblue and coral, samples from different omics layers (16 non-IPH and 26 IPH for each omics layer) are denoted by red, blue and green borders. Considering the high consistency between the components of the three omics layers in the multi-omics model (see Figure $3 C$ ), three sample distributions for different data layers were combined into one plot. (B) Heatmap shows expression/abundance level of the selected genes $(n=10)$, proteins $(n=10)$, and peptides $(n=10)$ for the first component of multi-omics analysis. Expression values were z-normalized per row. (C) Heatmap shows correlations between components from the multi-omics model. (D) Distribution of the selected genes, proteins, and peptides on their corresponding components of multi-omics SPLS-DA. Elements are plotted according to their Pearson's Correlation Coefficients to the component 1 and 2 ( $n=30$ for genes; $n=50$ for proteins; $n=110$ for peptides), or 3 and 4 ( $n=125$ for genes; $n=100$ for proteins; $n=170$ for peptides). (E) GSOA on standardized gene set of multi-omics sPLS-DA. For each component, six highly ranked overrepresented terms are selectively shown. (F) Overlapping between standardized gene subset of single-omics (S) component 1 and 2, and multi-omics (M) component 1 and 2.

Given the functionally related signature members in integrative sPLS-DA component 1 , suggesting a shared regulatory basis, we performed regulatory analysis by iRegulon to identify transcription factors (TFs) driving this signature set and built a regulatory network of the IPH signature encompassing mRNAs, protein, peptides, and in silico identified TFs. As can be seen from Figure 4B, all the 22 nonredundant members of component 1 were targeted by the top 5 enriched TFs. MRNA of the highest-ranked TF, serum response factor (SRF), was significantly and sharply down-regulated in haemorrhaged plaque regions, mirroring the effects seen on most of the SRF connected network members. SRF signalling is tightly dependent on its cofactor, where myocardin family members drive locomotion and cell adhesion activities, while T-cell factors will promote proliferation. Hypergeometric testing using the reference dataset of Xie's study ${ }^{43}$ showed a clear overrepresentation of SRF-targeting network members in megakaryoblastic leukemia-1/2 (MKL1/2) regulated SRF-dependent genes ( $p$-value $=2.7 E-03)$, but not in ETS-domain protein 4 (ELK4) regulated SRF-dependent genes (Figure 4C). This points to the former as the dominant signalling pathway in non-IPH plaque, promoting actin cytoskeleton organization, locomotion and cell adhesion regulated by SRF in plaque. 
One of the SRF-regulated features with the strongest down-regulation in IPH compared to non-IPH, at gene expression level (Figure 4B), was microfibrilassociated glycoprotein 4 (MFAP4), which has been described to localize in vascular wall extracellular matrix fibres and to be involved in neointima formation. ${ }^{44}$ Immunohistochemical validation of MFAP4 presence in plaque sections from the same cohort (MaasHPS) indeed confirmed its reduced abundance in haemorrhaged plaques (Figure 4D-E). We also examined the MFAP4 gene expression and protein abundance in BiKE cohort, which showed a down-regulation in atherosclerotic arteries compared to normal or adjacent tissues, as well as in symptomatic patients compared to asymptomatic patients (Figure S5A). Moreover, based on GSEA, we observed significant negative normalized enrichment scores (NES) of the SRF regulated network members in BiKE cohort for the comparisons of HIST and CLIN, suggesting the general down-regulation of the network members in carotid plaques compared with normal or adjacent tissues, and in symptomatic patients compared with asymptomatic patients (Figure S5B), which is in line with the expression in Figure 4B. 

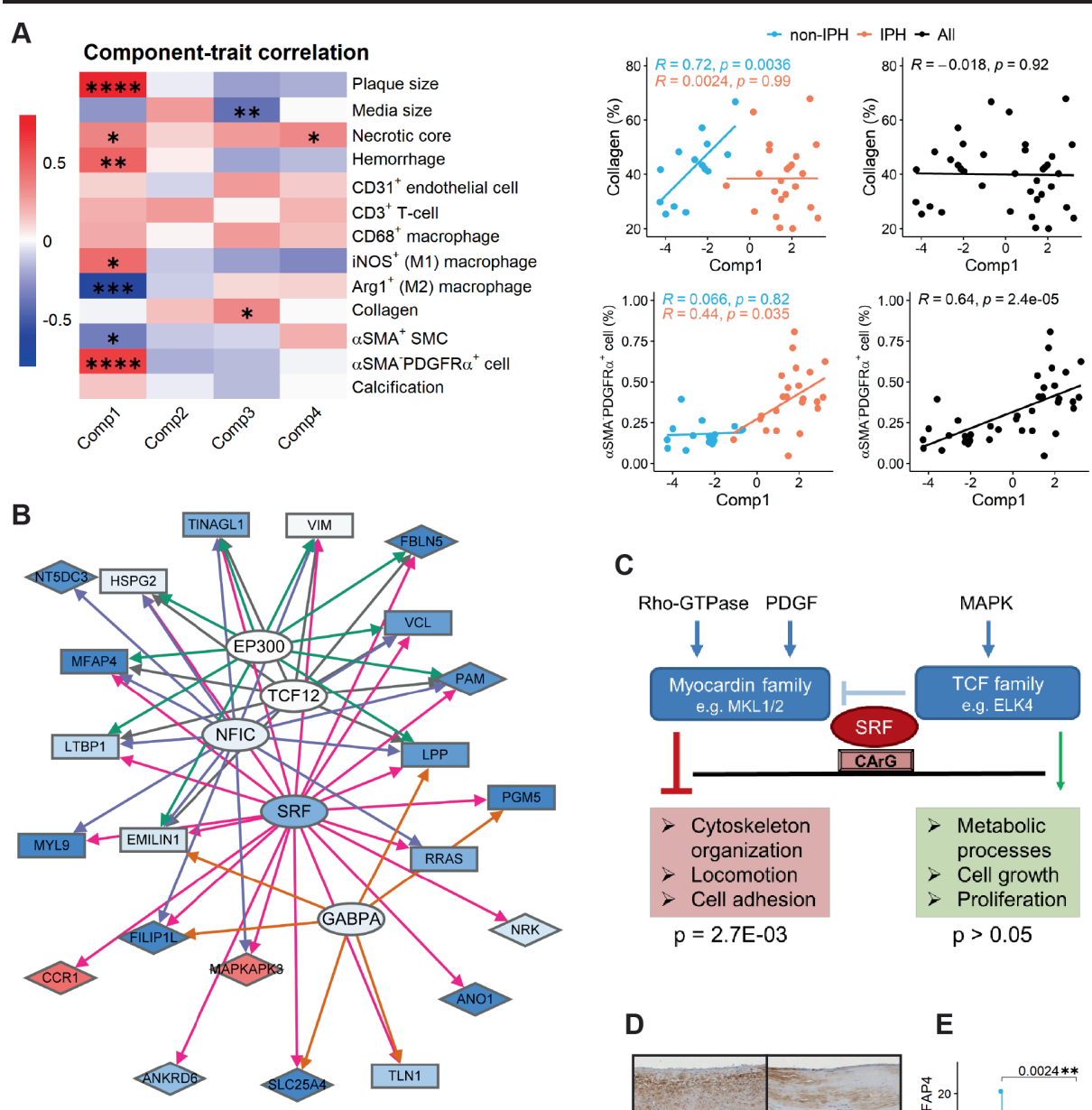

D
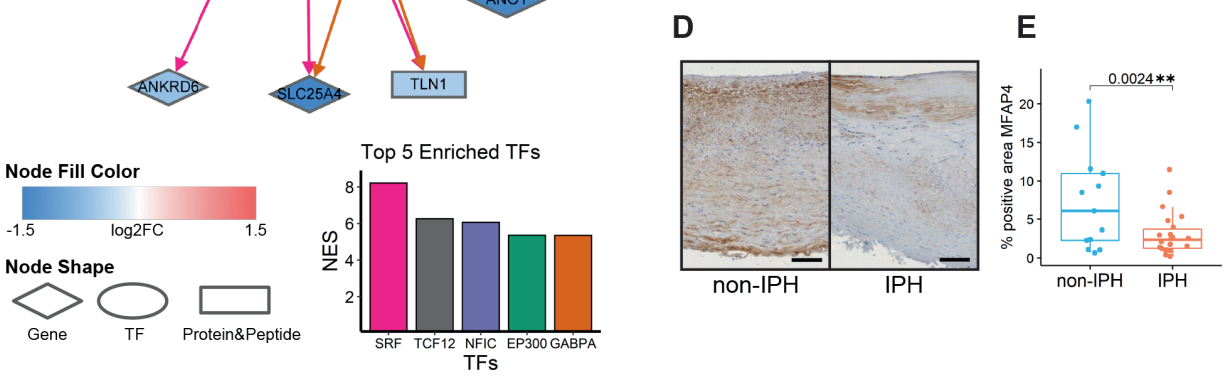

Figure 4. Biological extension of the results of the multi-omics analysis

(A) Correlation-based heatmap shows correlations and corresponding p-values (denoted as asterisk) between multi-omics component 1-4 (derived from the transcriptomic block) and plaque traits. Specifically, correlations of components with collagen content and $\alpha$ SMA-PDGFR $\alpha^{+}$fibroblast-like cell content are shown as scatter plots ( $n$ on-IPH, $n=16$; IPH, $n=26$; all plaques, $n=42$ ). (B) Transcription factors (TFs), targeting the elements from the first component of multi-omics sPLSDA based set of genes/proteins/peptides were extracted by iRegulon with default 
settings. Likely interactions of TFs with their downstream targets as obtained from iRegulon, are indicated by directed lines with different colours per TF. Genes, proteins and peptides, and TFs are depicted in different shapes; gene-level differential expression (log2 fold change) is shown by green-red gradient. The top 5 enriched TFs for multi-omics component 1 ranked by Normalized Enrichment Score (NES) are shown below, with the same colours as the directed lines in the network. (C) SRF network operating in plaques primarily steers the MKL1/2, not the ELK4 coregulated pathway. P-values were calculated by hypergeometric test. (D) Representative pictures of IHC for MFAP4 (brown area) in non-IPH and IPH plaques. Scale bar $=100 \mu \mathrm{m} ; 100 \times$ magnification. $(\mathrm{E})$ Differences of IHC-based MFAP4 positive area (\%) between paired non-IPH and IPH plaques (both $n=13$ ).

\section{Independent validation of findings from multi-omics analyses}

Finally, we assessed whether the IPH signature was able to discriminate low- from high-risk plaques in two independent mRNA cohorts in the public domain: GSE28829 ${ }^{40}$ ( $n=29$; mRNA; early vs advanced plaque) and GSE43292 ${ }^{4}$ ( $n=64$; mRNA; intact arterial tissue vs atheroma). Although the cohort setup differs from our cohort, carotid advanced plaque (GSE28829) and atheroma (GSE43292), respectively, both largely represent unstable plaque phenotypes (thin cap fibroatheroma and beyond). The main difference is in the control tissue with even lower risk than our non-haemorrhaged stable plaque: i.e., early-stage lesion (low risk) and non-lesioned arterial tissue, in the two validation cohorts, respectively, versus non-haemorrhaged stable plaque in our study, but we argued that our signature may still be valid for these broader disease trajectories.

Indeed, in both cohorts, the signature genes from integrative sPLS-DA component 1 (MULTI 1) were differentially expressed between control vs diseased artery (Figure 5A), with general downregulation in advanced plaque. Both the single-omics (SINGLE) and the multi-omics (MULTI 1, MULTI 1-4) signatures performed very well for the logistic regression and SVM classifiers, with AUCs of 0.95 in advanced vs early (GSE28829, Figure 5B and S6A). Of note, the first component of the multiomics model (MULTI 1 ) by itself already performed surprisingly well in stratifying the two plaque phenotypes for GSE28829, albeit not as good as the complete 4component gene set (MULTI 1-4, Figure 5B and S6A-B). Apparently, the first component is dominant in stratifying the validation cohorts, with a complementary contribution of components 2-4. Validation in GSE43292 did not show major differences in predictive power of the SINGLE, MULTI 1 and MULTI 1-4 gene sets; with overall high performance (AUCs $>0.8$ ) for all gene sets tested by logistic regression and SVM (Figure 5B and S6A). Decision tree classifiers performed less impressive than the other two classifiers (Figure S6B). Here, the MULTI 1-4 gene set appeared to be superior to the other models (Figure S6B), possibly owing to the Page | 92 
structure of the decision tree classifier, in which the splitting nodes can incorporate the independent features from distinct multi-omics components.

Finally, we validated the performance of the MULTI 1 signature in the BiKE cohort study, which includes both high-powered transcriptomics $(n=127)$ and proteomics datasets $(n=36)$ from CEA tissue. The MULTI 1 feature set performed well on both the transcriptomics and proteomics in segregating advanced plaque from healthy carotid tissue (histological, HIST; see Figure 5C). Interestingly, while both involving haemorrhaged plaques, our signature was even able to stratify, plaques from symptomatic versus asymptomatic patients based on their proteomics at a reasonable classification performance ( $A \cup C=0.80$ ), and to a lesser extent transcriptional profile ( $A \cup C=0.57$; clinical, CLIN; see Figure $5 C$ ). Indeed, all the MULTI 1 members overlap with MULTI 1-4 and SINGLE (Figure 5D), suggesting the MULTI 1 signature derived from the dominant component of the multi-omics model is essential for plaque classification. 


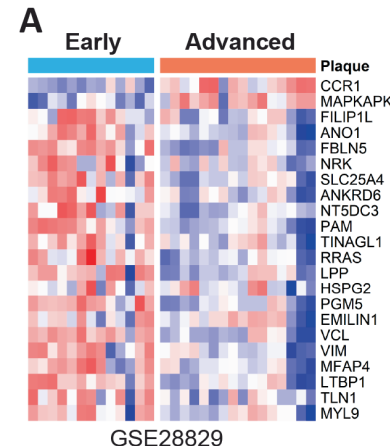

Normal

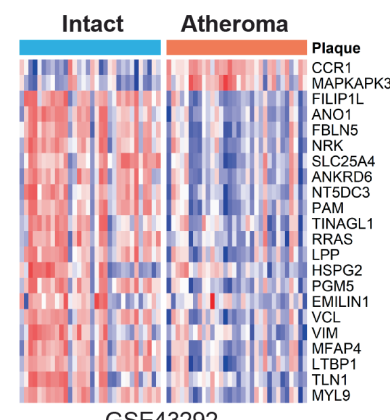

GSE43292

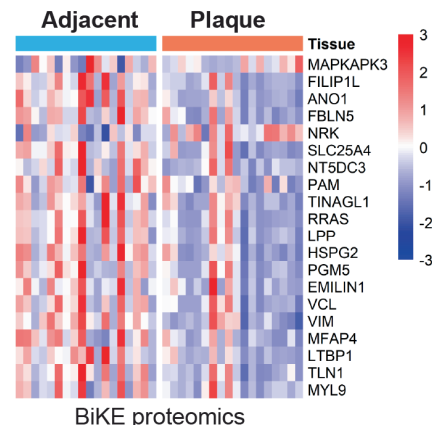

BiKE proteomics

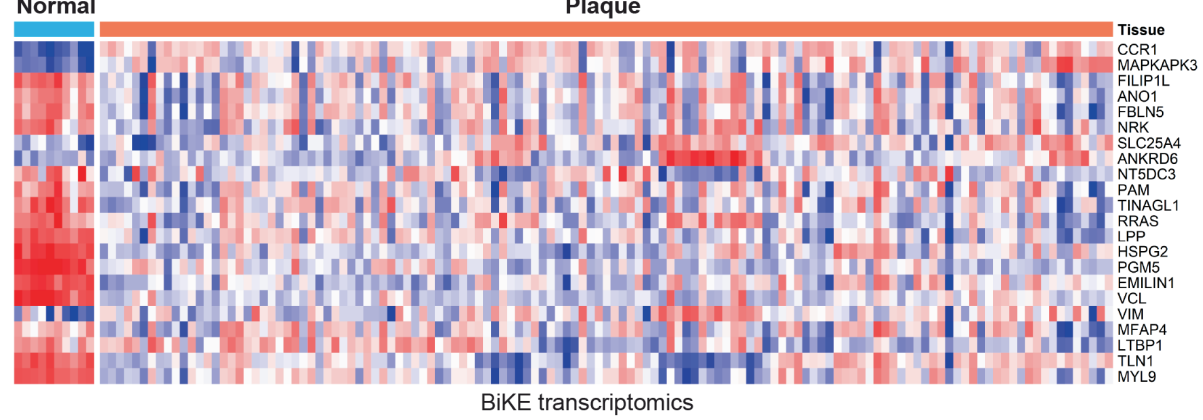

B
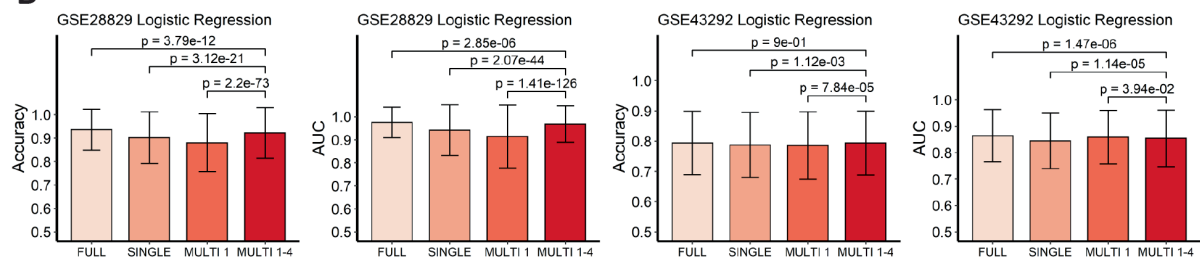

C
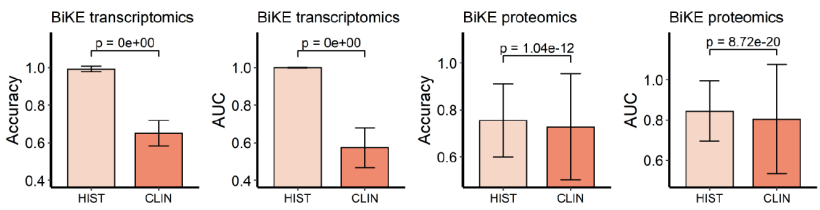

D

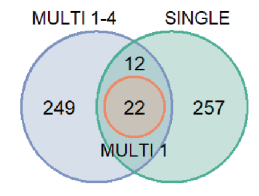

Figure 5. Validation of selected gene sets using independent cohorts

(A) Heatmaps shows expression/abundance pattern of the selected genes/proteins from component 1 by integrative sPLS-DA analysis (MULTI 1) in validation cohorts. For GSE28829, early ( $n=13$ ) vs advanced ( $n=16$ ) plaques; for GSE43292, intact arteries $(n=32)$ vs atheromata $(n=32)$; for BiKE proteomics, adjacent tissues $(n=$ 18 ) vs plaques $(n=18)$; for BiKE transcriptomics, normal tissues $(n=10)$ vs plaques $(n=127)$. Expression values were z-normalized per row. (B) Classification performance based on full data (FULL), single-omics (SINGLE), multi-omics 
component 1 (MULTI 1), and multi-omics component 1-4 (MULTI 1-4) feature subsets on two independent cohorts GSE28829 and GSE43292. (C) Classification performance of MULTI 1 feature subsets on BiKE cohort in distinguishing normal ( $\mathrm{n}$ $=10$ for transcriptomics; $n=18$ for proteomics) vs plaque ( $n=127$ for transcriptomics; $n=18$ for proteomics) based on histological characteristics (HIST), or asymptomatic ( $n=40$ for transcriptomics; $n=9$ for proteomics) vs symptomatic ( $n=87$ for transcriptomics; $n=9$ for proteomics) based on clinical events (CLIN). For Figure 5B-C, results were measured by accuracy and AUC using logistic regression, with stratified 5 -folds cross-validation and 1,000 random repeats. Results are presented as mean \pm SD. (D) Overlapping between feature sets SINGLE, MULTI 1, and MULTI 1-4. 


\section{DISCUSSION}

Elucidation of critical processes in the transition of low-risk into high-risk ruptureprone plaque in humans will pave the way for early diagnosis of, and targeted intervention in atherosclerosis-related cardiovascular diseases. Here we have deployed integrative analysis of transcriptomic, proteomic and peptidomic expression/abundance profiles of human carotid artery plaque, to build a multitethered prediction model for distinguishing low- from high-risk carotid artery lesions. The significance of this model was corroborated in multiple independent plaque cohort studies. Finally, based on this model, we constructed an SRF-driven regulatory gene/protein network overrepresented in extracellular matrix remodelling and interaction terms, that could serve as a starting point for the design of a targeted intervention.

Single-omics studies on human atherosclerotic plaque by us (this study, Goossens et al., Eijgelaar et al. $)^{22,45}$ and others ${ }^{4-6,8}$ have already shown the power of these high-throughput approaches for biomarker discovery and mechanistic studies. For instance, Ayari et al. have identified the CD163/HO-1 axis to be associated with iron-heme homeostasis in atherosclerotic plaque, using differential expression analysis on microarray data extracted from 68 carotid atheroma specimens ${ }^{4}$. However, single-omics approaches intrinsically fail to capture the complexity of biological systems as a whole, providing useful but incomplete information ${ }^{46,47}$. Moreover, genomic and peptide/protein expression/abundance in plaque were seen to show poor mutual correlation ${ }^{15}$. Consequently, genomics findings are not directly translatable to (pre)clinical application, while protein-based models generally are rather sparse, biased towards high abundance proteins, and lacking their regulatory context. This combined with the observation that both domains display distinct but complementary correlations with relevant clinical traits pleads in favour of integrative multi-omics analysis on atherosclerosis to have the best of both worlds, producing a robust, translatable model of disease progression.

This study illustrates the benefits of truly integrative omics analysis in terms of accuracy, visibility, and model interpretability. Our multi-omics prediction model achieved similar, if not higher, accuracy in stage classification as a single omics model. The prediction power of the selected features was successfully validated on several independent cohorts comparing healthy artery or early (stable) plaque vs advanced (unstable) plaque or atheroma. Even, the selected features performed reasonably well in stratifying plaques from symptomatic vs asymptomatic patients at protein level (BiKE proteomics), although plaques in both groups were having an advanced (unstable) phenotype. However, the true merit of integrative analysis is in the robustness of the model, its biological significance and the direct clinical translatability of network-contained proteins. Significances of overrepresented terms in the multi-omics signature outperformed those of the single-omics Page | 96 
signature, due to the strong mutual interaction of signature members per component. Interestingly, while the dominant component of the single and multiomics models showed considerable overlap, the auxiliary $2^{\text {nd }}$ to $4^{\text {th }}$ components of the single and multi-omics models differed substantially, with those of the latter being much more coherent and biologically meaningful. The dominant component in the multi-omics model was overrepresented in collagen metabolism and macrophage inflammation terms, reflecting peri-rupture modelling processes. The auxiliary components alluded to additional CVD relevant terms, such as lipid and lipoprotein metabolism, neutrophil responses and, again, tissue metabolism, mirroring early responses to haemorrhage and/or rupture. Additionally, we found that the BLVRB, which had been previously proposed by Matic et al. ${ }^{18}$ to define intraplaque haemorrhage, was also contained in our multi-omics model (component 4).

Literature search for the MULTI 1 elements indicated an overall relationship (15 of the total 22) to vascular smooth muscle function and inflammation in cardiovascular diseases (Table S3). Interestingly, the regulatory network based on the dominant component, identified SRF as the driving factor in peri-rupture remodelling. Despite the fact that a small media could not be excluded from the dissected tissue during the CEA surgery, the lack of correlation between the dominant component and the media size (Figure 4A) indicated the SRF-driven network was only associated with intimal not medial processes related to IPH. SRF is a MADS family type transcription factor, which acts by binding CArG box motifs. The relevance of SRF for cardiovascular health is well documented ${ }^{48,49}$. In cardiac and vascular smooth muscle cells, it was seen to drive phenotypic switch by regulating contractile gene program $\mathrm{s}^{50}$. In endothelial cells, SRF is essential for VEGF induced cell signalling and angiogenesis, and thus endothelial dysfunction ${ }^{51}$. Moreover, SRF mediates cellular lipid and glucose responses by controlling LXRB gene expression, modulating these metabolic sensors ${ }^{52}$. While a direct role in ischemic heart disease is likely, experimental data to underpin this notion is lacking.

Mechanistically, SRF transcriptional responses depend critically on its coactivator. The major coactivator classes are T-cell factors (e.g. TCF21 ${ }^{53}$, ELK1/2), which are responsible for renin-angiotensin-aldosterone system (RAAS) activated mechanosensing, and myocardin-family members (e.g. MRTF), which transduces mothers against decapentaplegic homolog (SMAD) and Rho-associated kinase responses ${ }^{54}$. As pointed out by Gualdrini et al., competition between T-cell and myocardin family members for SRF will skew its responses towards antagonistic proliferative and contractile programs of gene expression, respectively ${ }^{55}$. Our data seem to plead for MKL1/2-SRF signalling as the major dysregulated axis in IPH plaque, suggesting that remodelling responses will prevail. 
Although our integrative multi-omics approach holds promise, it has a number of limitations. First, this study was based on a moderately dimensioned cohort study containing CEA tissues from male patients and may therefore only partly reflect the population's diversity in disease risk profile as well as pathogenesis. The fact that we were able to confirm the validity of our selected genes in multi-omics model in several other cohorts, however indicates that our findings have a wider scope. Second, the multi-omics approach was only based on genes, proteins and peptides. Incorporation of metabolomics and lipidomics into the multi-omics signature may further strengthen the model and deepen our insights into key processes in this clinically relevant stage transition ${ }^{56,57}$.

\section{CONCLUSIONS}

In conclusion, our study underpins the added value of integrative multi-omics analysis to a single-omics study of human atherosclerosis. Comparing a single- and multi-omics analysis of human carotid atherosclerotic plaque, we showed that while both approaches perform excellently in segregating low- from high-risk plaques, the latter provides much deeper insight into critical processes taking place prior to or just after plaque rupture in humans. Moreover, our integrative multiomics approach revealed an SRF driven gene/protein network, associated with this phase transition, that could guide efforts to the design of new treatments to promote non-expansive plaque healing.

\section{DATA AVAILABILITY}

All microarray data are available in public data repositories. MaasHPS transcriptomics can be accessed from the Gene Expression Omnibus (GEO, https://www.ncbi.nlm.nih.gov/geo/) with accession number GSE163154. BiKE transcriptomics can be accessed from the GEO with accession number GSE21545. The other two validation data can be accessed from the GEO with accession number GSE43292 and GSE28829.

\section{AUTHOR CONTRIBUTIONS}

$\mathrm{HJ}$ conceived the study, processed the data, performed the computational analyses and drafted and revised the manuscript with input from all co-authors. CJJMS and BMEM performed the CEA surgery, WE, MM and MJAPD collected the plaque samples. PJ performed proteomics and peptidomics experimental analysis. 
Immunostaining was performed by JCS, KvK, OW, GEF, MJJG and other experienced colleagues. MM helped in data analysis and multi-omics data alignment. JK, ES, MK and CTAE gave input in the data analysis strategies. $\mathrm{LM}$ and $\mathrm{UH}$ performed analysis on the BiKE cohort dataset. JCS, PG, LM, MM and OW offered critical revision. EALB conceived and supervised the study, provided funding and critical feedback at all stages.

\section{ACKNOWLEDGEMENTS}

This work has been supported by the China Scholarship Council (Grant No. 201609120004) to Han Jin, the Marie Skłodowska-Curie Actions (MSCA-IF-2020, Grant No. 661099 AtheroMph Proliferation) to Pieter Goossens, the European Research Area Network on Cardiovascular Diseases (ERA-CVD and Dutch Heart Foundation, Grant No. JTC-2017t100 AtheroMacHete) to Pieter Goossens and Erik A.L. Biessen, the Dutch Heart Foundation (NWO, Grant No. 2016T060 and 2020T042) to Judith C. Sluimer and Pieter Goossens, the NWO VIDI fellowship (Grant No. 0.16.186.364), and a Leducq transatlantic network grant (Grant No. 15CVD04) to Judith C. Sluimer, two grants from the Centre for Translational Molecular Medicine (CTMM): Circulating Cells to Marco Manca, and the Plaque at RISK (PARISK) to Mat J.A.P. Daemen. The BiKE study and Ljubica Matic have been supported with funding from the Swedish Research Council (VR, 201902027), Swedish Heart-Lung Foundation (HLF, 20200621, 20200520, 20180244, 201602877, 20180247), Swedish Society for Medical Research (SSMF, P13-0171). Ljubica Matic also acknowledges funding from Sven and Ebba-Christina Hagberg, Tore Nilsson's, Magnus Bergvall's and Karolinska Institute research (KI Fonder) and doctoral education (KID) foundations. We thank Jennifer Campbell, Wade Hines, Mahesh Hulkarni, Moria Lynch, Manny Paniagua, Mahadevan Sethuraman from BG Medicine, Inc., Waltham, MA, USA for their help in proteomics experiment. We also thank Veronica Herías and Clairy Dinjens from Maastricht UMC+ for their assistance in analyzing plaque morphology.

Conflict of interest: none declared. 


\section{REFERENCES}

1. Song $\mathrm{P}$, Fang Z, Wang $\mathrm{H}$, et al. Global and regional prevalence, burden, and risk factors for carotid atherosclerosis: a systematic review, meta-analysis, and modelling study. Lancet Glob Heal. 2020;8(5):e721-e729.

2. Virmani R, Burke AP, Farb A, Kolodgie FD. Pathology of the Vulnerable Plaque. J Am Coll Cardiol. 2006;47(8 SUPPL.):C13-C18.

3. Libby P, Ridker PM, Hansson GK. Progress and challenges in translating the biology of atherosclerosis. Nature. 2011;473(7347):317-325.

4. Ayari $\mathrm{H}$, Bricca $\mathrm{G}$. Identification of two genes potentially associated in ironheme homeostasis in human carotid plaque using microarray analysis. J Biosci. 2013;38(2):311-315.

5. de Kleijn DPV, Moll FL, Hellings WE, et al. Local Atherosclerotic Plaques Are a Source of Prognostic Biomarkers for Adverse Cardiovascular Events. Arterioscler Thromb Vasc Biol. 2010;30(3):612-619.

6. Folkersen L, Persson J, Ekstrand J, et al. Prediction of Ischemic Events on the Basis of Transcriptomic and Genomic Profiling in Patients Undergoing Carotid Endarterectomy. Mol Med. 2012;18(4):669-675.

7. Perisic L, Aldi S, Sun Y, et al. Gene expression signatures, pathways and networks in carotid atherosclerosis. J Intern Med. 2016;279(3):293-308.

8. Diez D, Wheelock ÅM, Goto S, et al. The use of network analyses for elucidating mechanisms in cardiovascular disease. Mol Biosyst. 2010;6(2):289-304.

9. Chai JT, Ruparelia N, Goel A, et al. Differential Gene Expression in Macrophages From Human Atherosclerotic Plaques Shows Convergence on Pathways Implicated by Genome-Wide Association Study Risk Variants. Arterioscler Thromb Vasc Biol. 2018;38(11):2718-2730.

10. Chinetti-Gbaguidi G, Daoudi M, Rosa M, et al. Human Alternative Macrophages Populate Calcified Areas of Atherosclerotic Lesions and Display Impaired RANKL-Induced Osteoclastic Bone Resorption Activity. Circ Res. 2017;121(1):19-30.

11. Cochain C, Vafadarnejad E, Arampatzi P, et al. Single-Cell RNA-Seq Reveals the Transcriptional Landscape and Heterogeneity of Aortic Macrophages in Murine Atherosclerosis. Circ Res. 2018;122(12):1661-1674.

12. Winkels $\mathrm{H}$, Ehinger $\mathrm{E}$, Vassallo $\mathrm{M}$, et al. Atlas of the Immune Cell Repertoire in Mouse Atherosclerosis Defined by Single-Cell RNA-Sequencing and Mass Cytometry. Circ Res. 2018;122(12):1675-1688.

13. Kim K, Shim D, Lee JS, et al. Transcriptome Analysis Reveals Nonfoamy Rather Than Foamy Plaque Macrophages Are Proinflammatory in Atherosclerotic Murine Models. Circ Res. 2018;123(10):1127-1142.

14. Rogers S, Girolami M, Kolch W, et al. Investigating the correspondence between transcriptomic and proteomic expression profiles using coupled 
cluster models. Bioinformatics. 2008;24(24):2894-2900.

15. Ghazalpour A, Bennett B, Petyuk VA, et al. Comparative Analysis of Proteome and Transcriptome Variation in Mouse. Snyder M, ed. PLoS Genet. 2011;7(6):e1001393.

16. Bersanelli M, Mosca $E$, Remondini $D$, et al. Methods for the integration of multi-omics data: mathematical aspects. BMC Bioinformatics. 2016;17(S2):S15.

17. Hasin Y, Seldin M, Lusis A. Multi-omics approaches to disease. Genome Biol. 2017;18(1):83.

18. Matic LP, Jesus Iglesias M, Vesterlund M, et al. Novel Multiomics Profiling of Human Carotid Atherosclerotic Plaques and Plasma Reveals Biliverdin Reductase B as a Marker of Intraplaque Hemorrhage. JACC Basic to Transl Sci. 2018;3(4):464-480.

19. Langley SR, Willeit K, Didangelos A, et al. Extracellular matrix proteomics identifies molecular signature of symptomatic carotid plaques. J Clin Invest. 2017;127(4):1546-1560.

20. Tomas L, Edsfeldt A, Mollet IG, et al. Altered metabolism distinguishes highrisk from stable carotid atherosclerotic plaques. Eur Heart J. 2018;39(24):2301-2310.

21. Buescher JM, Driggers EM. Integration of omics: more than the sum of its parts. Cancer Metab. 2016;4(1):4.

22. Goossens P, Gijbels MJJ, Zernecke A, et al. Myeloid type I interferon signaling promotes atherosclerosis by stimulating macrophage recruitment to lesions. Cell Metab. 2010;12(2):142-153.

23. Michel JB, Virmani R, Arbustini E, Pasterkamp G. Intraplaque haemorrhages as the trigger of plaque vulnerability. Eur Heart J. 2011;32(16):1977-1985.

24. Hellings WE, Peeters W, Moll FL, et al. Composition of carotid atherosclerotic plaque is associated with cardiovascular outcome: A prognostic study. Circulation. 2010;121(17):1941-1950.

25. Rohart F, Gautier B, Singh A, Lê Cao K-A. mixOmics: An R package for 'omics feature selection and multiple data integration. Schneidman D, ed. PLOS Comput Biol. 2017;13(11):e1005752.

26. Juhasz $\mathrm{P}$, Lynch $\mathrm{M}$, Sethuraman $\mathrm{M}$, et al. Semi-targeted plasma proteomics discovery workflow utilizing two-stage protein depletion and off-line LCMALDI MS/MS. J Proteome Res. 2011;10(1):34-45.

27. Vandesompele J, De Preter K, Pattyn F, et al. Accurate normalization of realtime quantitative RT-PCR data by geometric averaging of multiple internal control genes. Genome Biol. 2002;3(7).

28. Troyanskaya $\mathrm{O}$, Cantor $\mathrm{M}$, Sherlock $\mathrm{G}$, et al. Missing value estimation methods for DNA microarrays. Bioinformatics. 2001;17(6):520-525.

29. Chung D, Keles S. Sparse Partial Least Squares Classification for High 
Dimensional Data. Stat Appl Genet Mol Biol. 2010;9(1).

30. Lê Cao K-A, Boitard S, Besse P. Sparse PLS discriminant analysis: biologically relevant feature selection and graphical displays for multiclass problems. BMC Bioinformatics. 2011;12(1):253.

31. Tibshirani R. Regression Shrinkage and Selection Via the Lasso. J R Stat Soc Ser B. 1996;58(1):267-288.

32. Günther OP, Shin H, Ng RT, et al. Novel Multivariate Methods for Integration of Genomics and Proteomics Data: Applications in a Kidney Transplant Rejection Study. Omi A J Integr Biol. 2014;18(11):682-695.

33. Barker $M$, Rayens W. Partial least squares for discrimination. J Chemom. 2003;17(3):166-173.

34. Pérez-Enciso $M$, Tenenhaus $M$. Prediction of clinical outcome with microarray data: A partial least squares discriminant analysis (PLS-DA) approach. Hum Genet. 2003;112(5-6):581-592.

35. Nguyen D V., Rocke DM. Tumor classification by partial least squares using microarray gene expression data. Bioinformatics. 2002;18(1):39-50.

36. Singh A, Shannon CP, Gautier B, et al. DIABLO: an integrative approach for identifying key molecular drivers from multi-omics assays. Birol I, ed. Bioinformatics. 2019;35(17):3055-3062.

37. Yu G, Wang LG, Han Y, He QY. ClusterProfiler: An R package for comparing biological themes among gene clusters. Omi A J Integr Biol. 2012;16(5):284287.

38. Yu G, He QY. ReactomePA: An R/Bioconductor package for reactome pathway analysis and visualization. Mol Biosyst. 2016;12(2):477-479.

39. Janky R, Verfaillie A, Imrichová $\mathrm{H}$, et al. iRegulon: From a Gene List to a Gene Regulatory Network Using Large Motif and Track Collections. PLoS Comput Biol. 2014;10(7):e1003731.

40. Döring Y, Manthey HD, Drechsler M, et al. Auto-Antigenic Protein-DNA Complexes Stimulate Plasmacytoid Dendritic Cells to Promote Atherosclerosis. Circulation. 2012;125(13):1673-1683.

41. Virmani R, Kolodgie FD, Burke AP, Farb A, Schwartz SM. Lessons From Sudden Coronary Death. Arterioscler Thromb Vasc Biol. 2000;20(5):12621275.

42. González I, Cao K-AL, Davis MJ, Déjean S. Visualising associations between paired 'omics' data sets. BioData Min. 2012;5(1):19.

43. Xie L. MKL1/2 and ELK4 co-regulate distinct serum response factor (SRF) transcription programs in macrophages. BMC Genomics. 2014;15(1):301.

44. Schlosser A, Pilecki B, Hemstra LE, et al. MFAP4 Promotes Vascular Smooth Muscle Migration, Proliferation and Accelerates Neointima Formation. Arterioscler Thromb Vasc Biol. 2016;36(1):122-133.

45. Eijgelaar WJ, Horrevoets AJG, Bijnens APJJ, Daemen MJAP, Verhaegh WFJ. 
Equivalence testing in microarray analysis: Similarities in the transcriptome of human atherosclerotic and nonatherosclerotic macrophages. Physiol Genomics. 2010;41(3):212-223.

46. Palsson B, Zengler K. The challenges of integrating multi-omic data sets. Nat Chem Biol. 2010;6(11):787-789.

47. Gomez-Cabrero D, Abugessaisa I, Maier D, et al. Data integration in the era of omics: current and future challenges. BMC Syst Biol. 2014;8 Suppl 2(Suppl 2):I1.

48. Miano JM, Ramanan N, Georger MA, et al. Restricted inactivation of serum response factor to the cardiovascular system. Proc Natl Acad Sci U S A. 2004;101(49):17132-17137.

49. Doi $\mathrm{H}$, Iso $\mathrm{T}$, Yamazaki $\mathrm{M}$, et al. HERP1 inhibits myocardin-induced vascular smooth muscle cell differentiation by interfering with SRF binding to CArG box. Arterioscler Thromb Vasc Biol. 2005;25(11):2328-2334.

50. Xia XD, Zhou Z, Yu X hua, Zheng XL, Tang CK. Myocardin: A novel player in atherosclerosis. Atherosclerosis. 2017;257:266-278.

51. Franco CA, Blanc J, Parlakian A, et al. SRF selectively controls tip cell invasive behavior in angiogenesis. Dev. 2012;140(11):2321-2333.

52. Nilsson M, Dahlman-Wright K, Karelmo C, Ebeling M, Gustafsson J-A, Steffensen KR. Elk1 and SRF transcription factors convey basal transcription and mediate glucose response via their binding sites in the human LXRB gene promoter. Nucleic Acids Res. 2007;35(14):4858-4868.

53. Nagao M, Lyu Q, Zhao Q, et al. Coronary Disease-Associated Gene TCF21 Inhibits Smooth Muscle Cell Differentiation by Blocking the MyocardinSerum Response Factor Pathway. Circ Res. 2020;126(4):517-529.

54. Iwasaki K, Hayashi K, Fujioka T, Sobue K. Rho/Rho-associated kinase signal regulates myogenic differentiation via myocardin-related transcription factor-A/Smad-dependent transcription of the Id3 gene. J Biol Chem. 2008;283(30):21230-21241.

55. Gualdrini F, Esnault C, Horswell S, Stewart A, Matthews N, Treisman R. SRF Co-factors Control the Balance between Cell Proliferation and Contractility. Mol Cell. 2016;64(6):1048-1061.

56. Blazier AS, Papin JA. Integration of expression data in genome-scale metabolic network reconstructions. Front Physiol. 2012;3:299.

57. Huang S, Chaudhary K, Garmire LX. More Is Better: Recent Progress in MultiOmics Data Integration Methods. Front Genet. 2017;8:84. 


\section{SUPPLEMENTAL DATA}
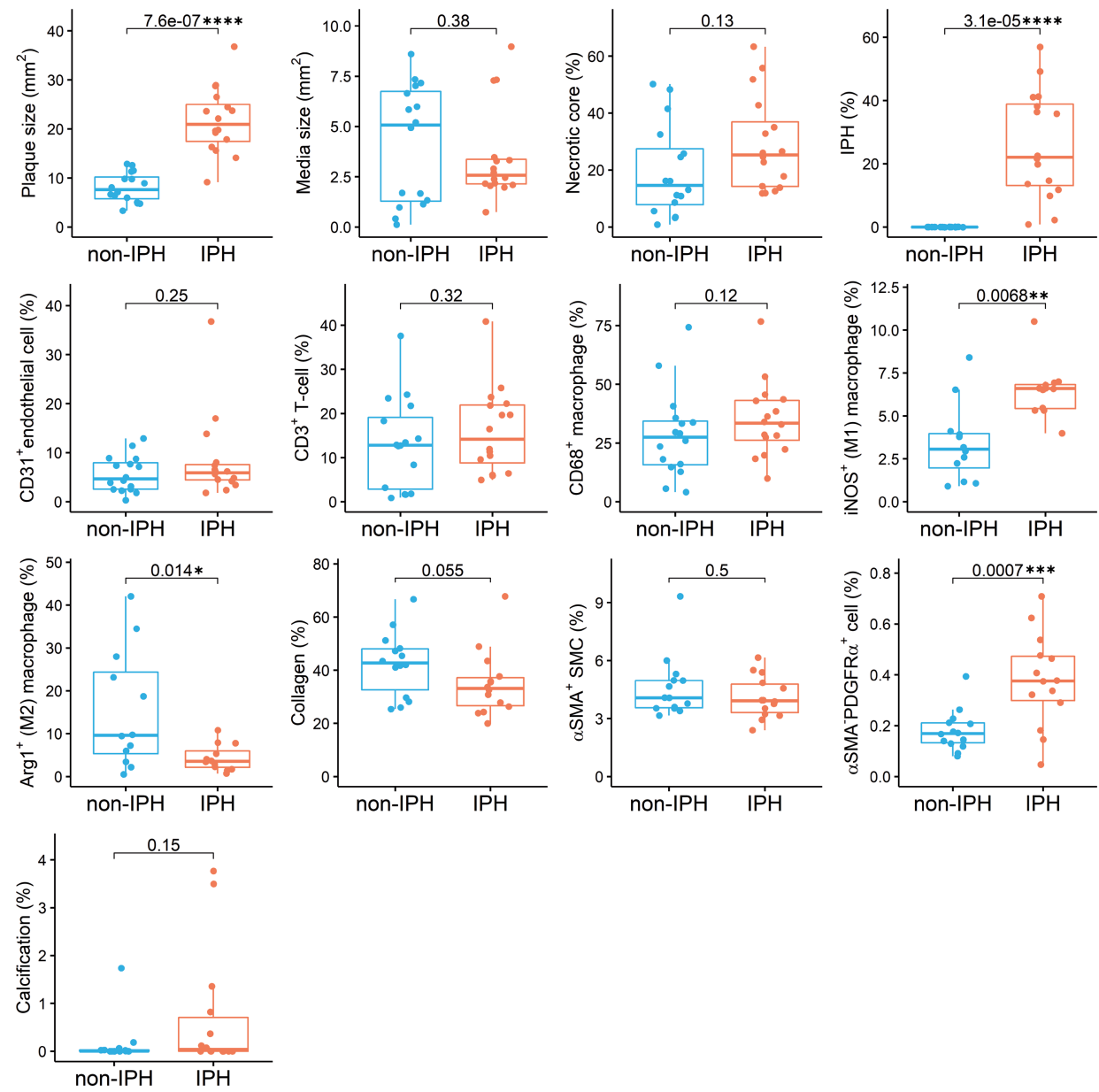

Figure S1. Distribution of plaque traits

Distribution of plaque traits between paired non-IPH and IPH (both $n=16$ ) samples. 


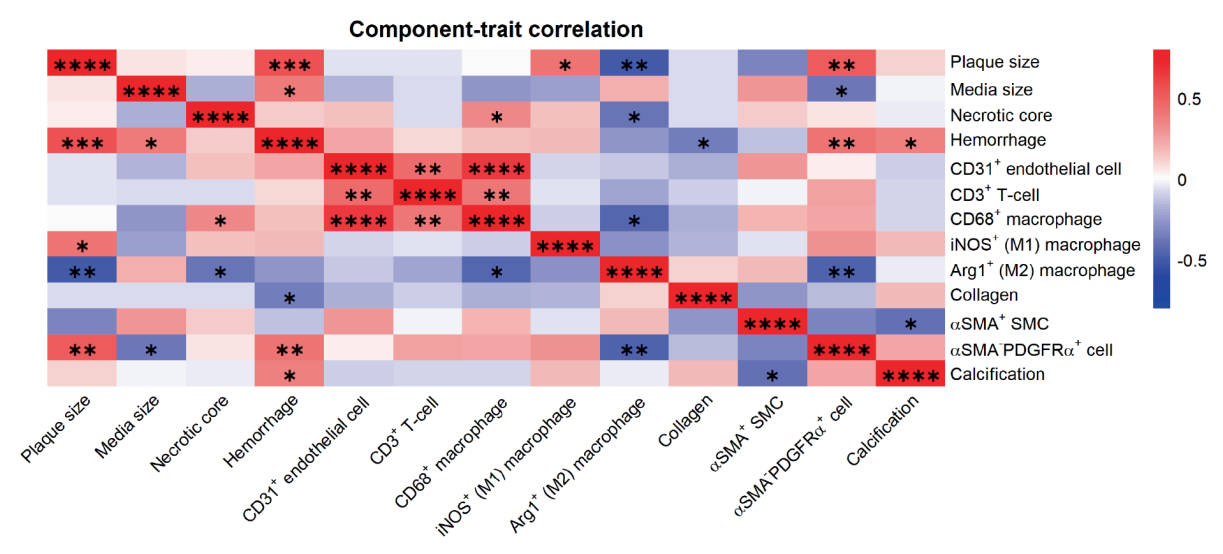

Figure S2. Correlations between plaque traits

Heatmap shows correlations between plaque traits. 

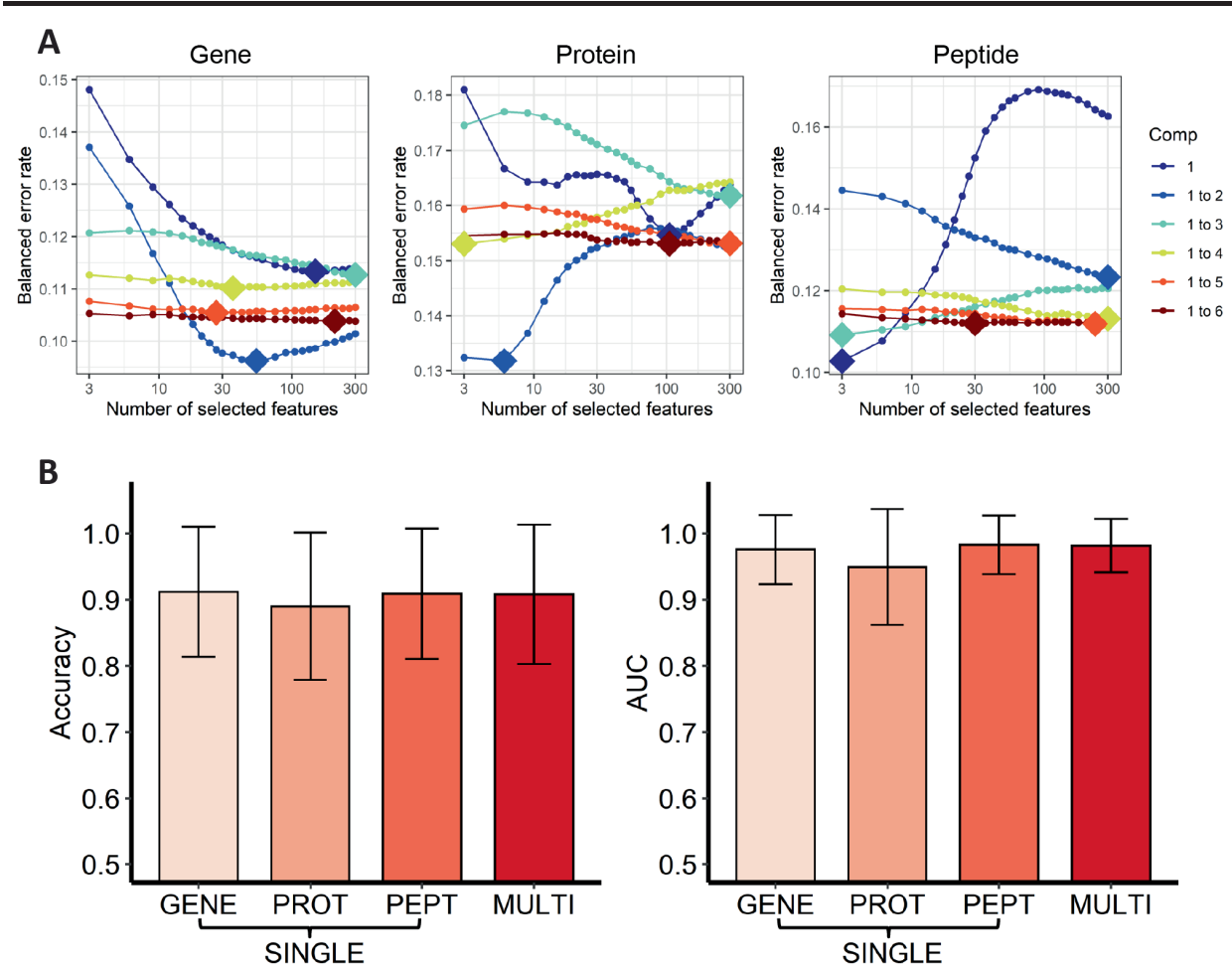

Figure S3. Parameter optimization

(A) Balanced Error rate of training models under different parameter settings for single-omics SPLS-DA on transcriptomics, proteomics, and peptidomics. A range of features from 3 to 300 (from 3 to 30 in steps of 3, from 30 to 60 in steps of 6, from 60 to 150 in the step of 15, and from 150 to 300 in the step of 30) was set for each component. Results were calculated with stratified 5-fold cross-validation and 1,000 random repeats. (B) Performance of the prediction models under the optimal parameter settings. Results were measured by accuracy and AUC, with stratified 5fold cross-validation and 10,000 random repeats. Results are presented as mean \pm SD. GENE, PROT, PEPT stand for the results of single sPLS-DA (SINGLE) based on transcriptomics, proteomics, and peptidomics, respectively. MULTI represents the results of integrative SPLS-DA on the three omics. 


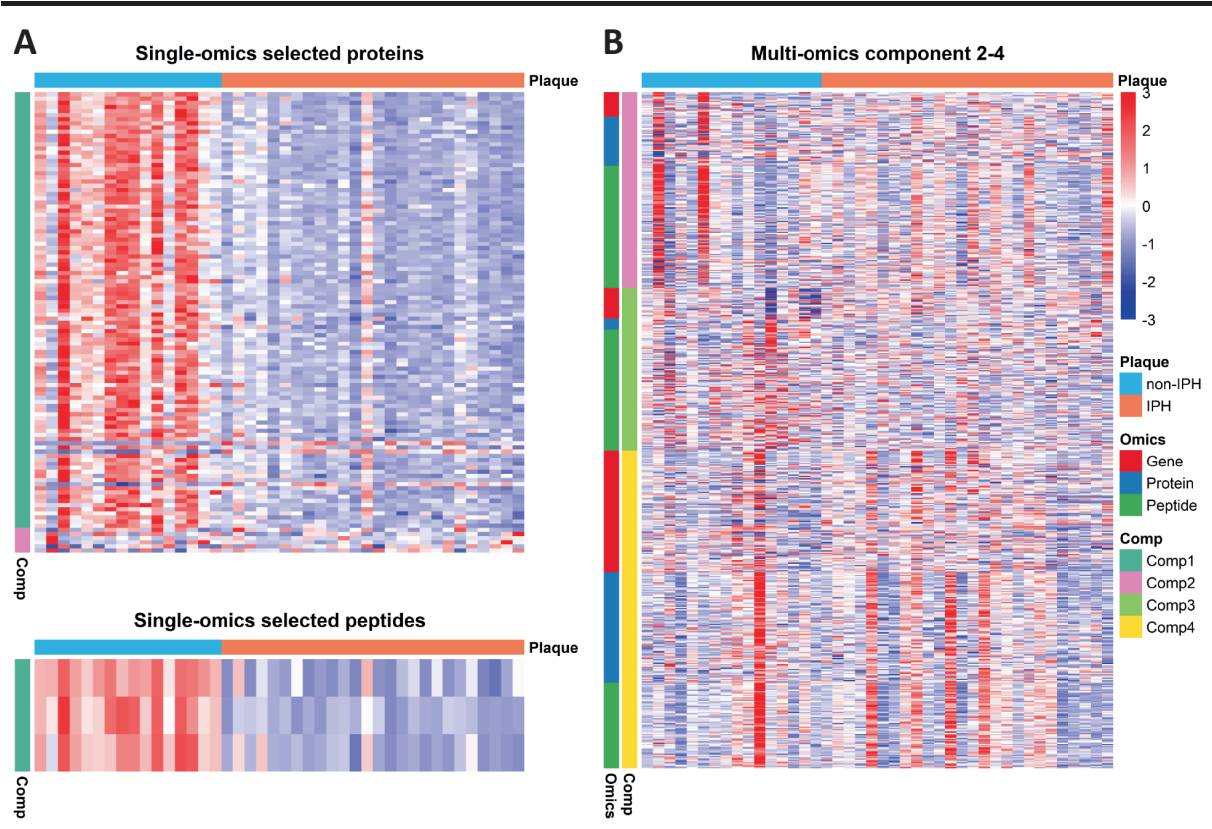

Figure S4. Heatmap of the selected features

(A) Heatmaps show the expression level of the selected proteins from proteomics and peptides from peptidomics by single-omics analysis. (B) Heatmap shows the expression level of the selected features for component 2-4 of multi-omics analysis. Expression values were z-normalized per row. 
A
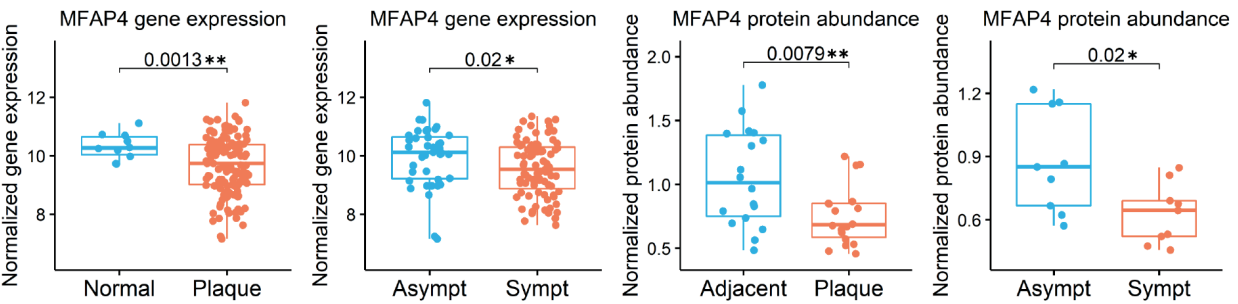

B

BiKE Transcriptomics Normal vs Plaque

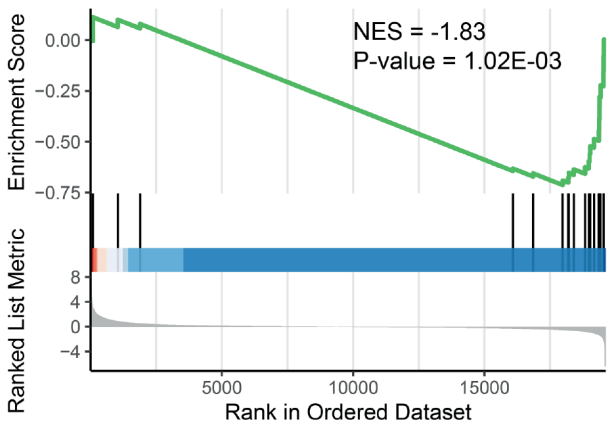

BiKE Proteomics Adjacent vs Plaque

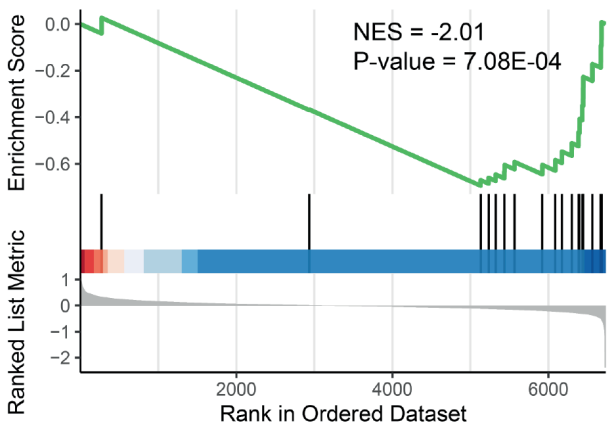

BiKE Transcriptomics Asympt vs Sympt

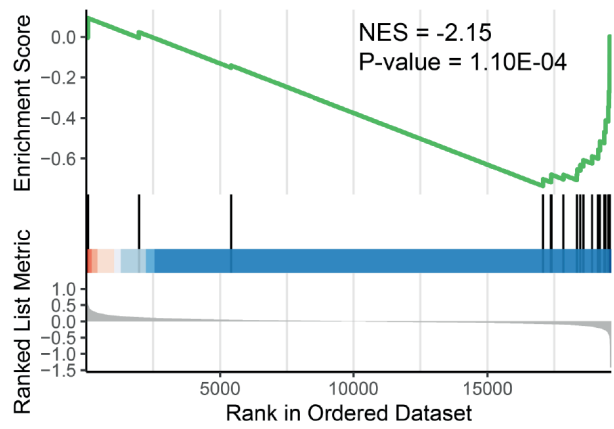

BiKE Proteomics Asympt vs Sympt

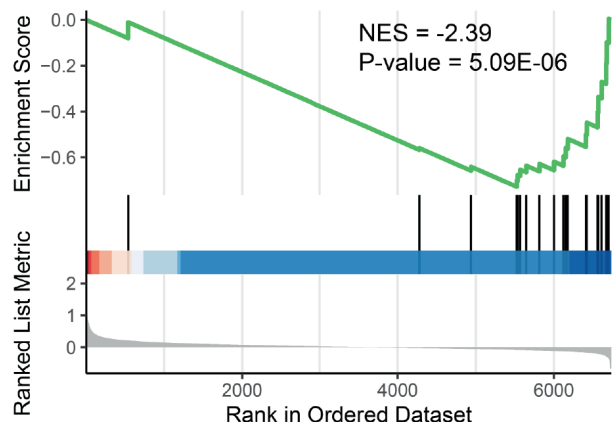

Figure S5. Validation of MFAP4 and other SRF targeting genes in BiKE cohort

(A) Gene expression and protein abundance of MFAP4 in BiKE cohort. The levels of gene expression were compared between normal (Normal, $n=10$ ) and atherosclerotic arteries (Plaque, $n=127$ ), and between asymptomatic (Asympt, $n=$ 40) and symptomatic (Sympt, $n=87$ ) patients. The levels of protein abundance were compared between adjacent healthy segments (Adjacent, $n=18$ ) and atherosclerotic segments (Plaque, $n=18$ ), and between asymptomatic (Asympt, $n$ =9) and symptomatic (Sympt, $n=9$ ) patients. Results are shown as box and whiskers plots with the same setting in Figure S1. P-values were calculated using a two-tailed Wilcoxon rank-sum test or Student's t-test. (B) GSEA of the SRF targeting network members (see Figure 4B) on BiKE cohort. Genes and proteins in BiKE 
transcriptomics and proteomics were ranked based on the comparisons in Figure S5A and were used as the GSEA background. NES, normalized enrichment score. 
A
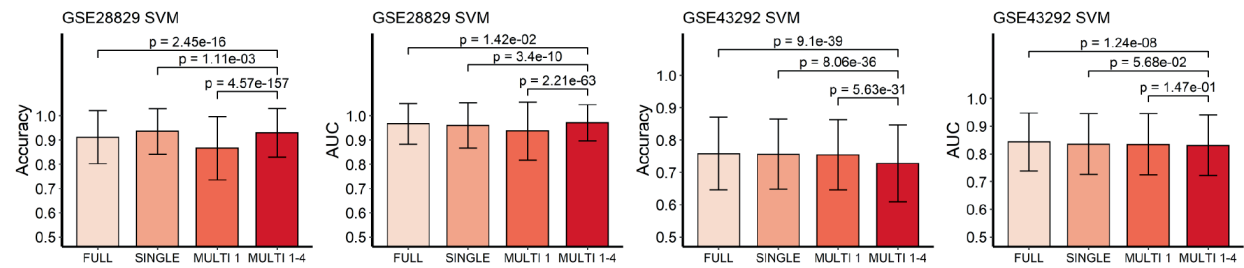

B
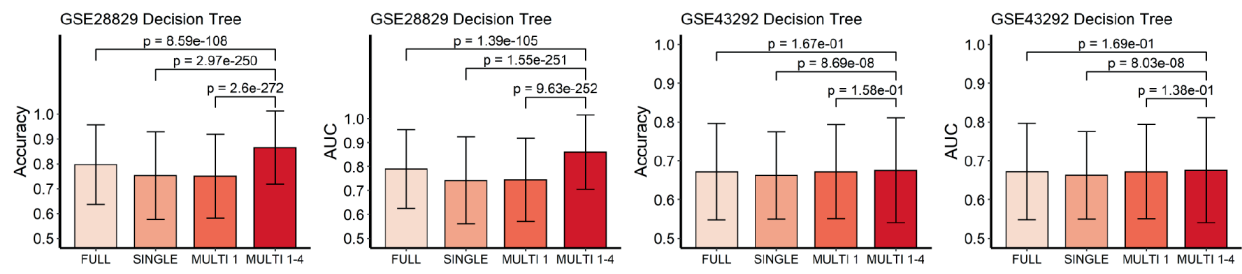

Figure S6. Validation of selected gene sets in independent cohorts

Classification performance based on full data (FULL), single-omics (SINGLE), multiomics component 1 (MULTI 1), and multi-omics component 1-4 (MULTI 1-4) feature subsets on two independent cohorts GSE28829 and GSE43292. Results were measured by accuracy and AUC using SVM (A) and decision tree (B), respectively, with stratified 5 -folds cross-validation and 1,000 random repeats. Results are presented as mean \pm SD. 


\begin{tabular}{|c|c|c|c|c|c|}
\hline Sample & Patient & Sample classification & Flanking sections & Age & Gender \\
\hline 1 & 1 & non-IPH & FCA & 78 & M \\
\hline 2 & 2 & non-IPH & FCA & 65 & $M$ \\
\hline 3 & 3 & non-IPH & $\mathrm{PIT}+\mathrm{FCA}$ & 78 & $M$ \\
\hline 4 & 5 & non-IPH & PIT & 83 & $\mathrm{M}$ \\
\hline 5 & 6 & non-IPH & FCA & 71 & $M$ \\
\hline 6 & 8 & non-IPH & FCA & 72 & $M$ \\
\hline 7 & 9 & non-IPH & IT & 80 & $\mathrm{M}$ \\
\hline 8 & 10 & non-IPH & FCA & 70 & $M$ \\
\hline 9 & 13 & non-IPH & FCA & 69 & $M$ \\
\hline 10 & 15 & non-IPH & FCA & 73 & $M$ \\
\hline 11 & 16 & non-IPH & FCA & 76 & $M$ \\
\hline 12 & 17 & non-IPH & FCA & 67 & $\mathrm{M}$ \\
\hline 13 & 18 & non-IPH & FCA & 66 & $M$ \\
\hline 14 & 20 & non-IPH & IT & 82 & $M$ \\
\hline 15 & 21 & non-IPH & FCA & 72 & $M$ \\
\hline 16 & 22 & non-IPH & FCA & 83 & $\mathrm{M}$ \\
\hline 17 & 1 & $\mathrm{IPH}$ & $\mathrm{IPH}$ & 78 & $\mathrm{M}$ \\
\hline 18 & 2 & $\mathrm{IPH}$ & $\mathrm{IPH}$ & 65 & $\mathrm{M}$ \\
\hline 19 & 3 & $\mathrm{IPH}$ & Ruptured & 78 & $M$ \\
\hline 20 & 4 & $\mathrm{IPH}$ & $\mathrm{IPH}$ & 64 & $\mathrm{M}$ \\
\hline 21 & 4 & $\mathrm{IPH}$ & $\mathrm{IPH}$ & 64 & $M$ \\
\hline 22 & 5 & $\mathrm{IPH}$ & $\mathrm{IPH}$ & 83 & $M$ \\
\hline 23 & 6 & $\mathrm{IPH}$ & $\mathrm{IPH}$ & 71 & $\mathrm{M}$ \\
\hline 24 & 8 & $\mathrm{IPH}$ & $\mathrm{IPH}$ & 72 & $M$ \\
\hline 25 & 9 & $\mathrm{IPH}$ & $\mathrm{IPH}$ & 80 & $M$ \\
\hline 26 & 10 & $\mathrm{IPH}$ & $\mathrm{IPH}$ & 70 & $\mathrm{M}$ \\
\hline 27 & 11 & $\mathrm{IPH}$ & $\mathrm{IPH}$ & 79 & $M$ \\
\hline 28 & 11 & $\mathrm{IPH}$ & $\mathrm{IPH}$ & 79 & $\mathrm{M}$ \\
\hline 29 & 12 & $\mathrm{IPH}$ & Ruptured & 78 & $M$ \\
\hline 30 & 13 & $\mathrm{IPH}$ & $\mathrm{IPH}$ & 69 & $M$ \\
\hline 31 & 14 & $\mathrm{IPH}$ & $\mathrm{IPH}$ & 76 & $\mathrm{M}$ \\
\hline 32 & 15 & $\mathrm{IPH}$ & IPH + Ruptured & 73 & $M$ \\
\hline 33 & 16 & $\mathrm{IPH}$ & IPH + Ruptured & 76 & $M$ \\
\hline
\end{tabular}


Chapter 3

\begin{tabular}{|l|l|l|l|l|l|}
\hline $\mathbf{3 4}$ & 17 & $\mathrm{IPH}$ & $\mathrm{IPH}$ & 67 & $\mathrm{M}$ \\
\hline $\mathbf{3 5}$ & 18 & $\mathrm{IPH}$ & $\mathrm{IPH}$ & 66 & $\mathrm{M}$ \\
\hline $\mathbf{3 6}$ & 19 & $\mathrm{IPH}$ & $\mathrm{IPH}$ & 64 & $\mathrm{M}$ \\
\hline $\mathbf{3 7}$ & 19 & $\mathrm{IPH}$ & $\mathrm{IPH}$ & 64 & $\mathrm{M}$ \\
\hline $\mathbf{3 8}$ & 20 & $\mathrm{IPH}$ & $\mathrm{IPH}$ & 82 & $\mathrm{M}$ \\
\hline $\mathbf{3 9}$ & 21 & $\mathrm{IPH}$ & $\mathrm{IPH}$ & 72 & $\mathrm{M}$ \\
\hline $\mathbf{4 0}$ & 22 & $\mathrm{IPH}$ & $\mathrm{IPH}+$ Ruptured & 83 & $\mathrm{M}$ \\
\hline $\mathbf{4 1}$ & 23 & $\mathrm{IPH}$ & $\mathrm{IPH}+$ Ruptured & 64 & $\mathrm{M}$ \\
\hline $\mathbf{4 2}$ & 24 & $\mathrm{IPH}$ & $\mathrm{IPH}$ & 67 & $\mathrm{M}$ \\
\hline
\end{tabular}

Table S1. Information of the patients. PIT/IT, (pathological) intimal thickening; FCA, fibrous cap atheroma; IPH, intraplaque haemorrhage. 


\begin{tabular}{|c|c|c|c|c|}
\hline & \multicolumn{3}{|c|}{ Single sPLS-DA } \\
\hline Component & Transcriptomics & Proteomics & Peptidomics \\
\hline Component 1 & 150 & 105 & 3 \\
\hline Component 2 & 54 & 6 & NA \\
\hline Component 3 & NA & NA & NA \\
\hline Component 4 & NA & NA & NA \\
\hline & & Integrative sPLS-DA \\
\hline Component & Transcriptomics & Proteomics & Peptidomics \\
\hline Component 1 & 10 & 10 & 10 \\
\hline Component 2 & 20 & 40 & 100 \\
\hline Component 3 & 25 & 10 & 100 \\
\hline Component 4 & 100 & 90 & 70 \\
\hline
\end{tabular}

Table S2. The optimal parameters for model building 
Chapter 3

\begin{tabular}{|l|l|l|}
\hline Gene symbol & PUBMED ID & Brief \\
\hline ANKRD6 & NA & NA \\
\hline ANO1 & $32894555 ; 28539652$ & Myocardial fibrosis \\
\hline CCR1 & 18088392 & Myocardial infarction \\
\hline EMILIN1 & $\begin{array}{l}22814752 ; 28545768 ; \\
25056700\end{array}$ & $\begin{array}{l}\text { Vascular smooth muscle cell, } \\
\text { extracellular matrix }\end{array}$ \\
\hline FBLN5 & $27089918 ; 27692634$ & Vascular inflammation \\
\hline FILIP1L & NA & NA \\
\hline HSPG2 & 10580178 & Coronary artery disease \\
\hline LPP & 12760907 & Smooth muscle cell \\
\hline LTBP1 & 25805620 & Cardiovascular development \\
\hline MAPKAPK3 & 23608535 & Fiber and cardiomyocyte \\
\hline MFAP4 & $26564819 ; 32856514$ & Vascular smooth muscle cell \\
\hline MYL9 & 22003410 & $\begin{array}{l}\text { Aging-associated cardiovascular } \\
\text { system }\end{array}$ \\
\hline NRK & 32330120 & Vascular inflammation \\
\hline NT5DC3 & NA & NA \\
\hline PAM & NA & NA \\
\hline PGM5 & NA & NA \\
\hline RRAS & NA & NA \\
\hline SLC25A4 & $30165862 ; 23401503$ & Cardiomyopathy \\
\hline TINAGL1 & NA & Nascular inflammation \\
\hline TLN1 & 30888838 & \\
\hline VCL & 32516855 & Coronary artery disease \\
\hline VIM & 30451917 & Cardiomyopathy \\
\hline
\end{tabular}

Table S3. Literature search for the MULTI 1 selected genes 




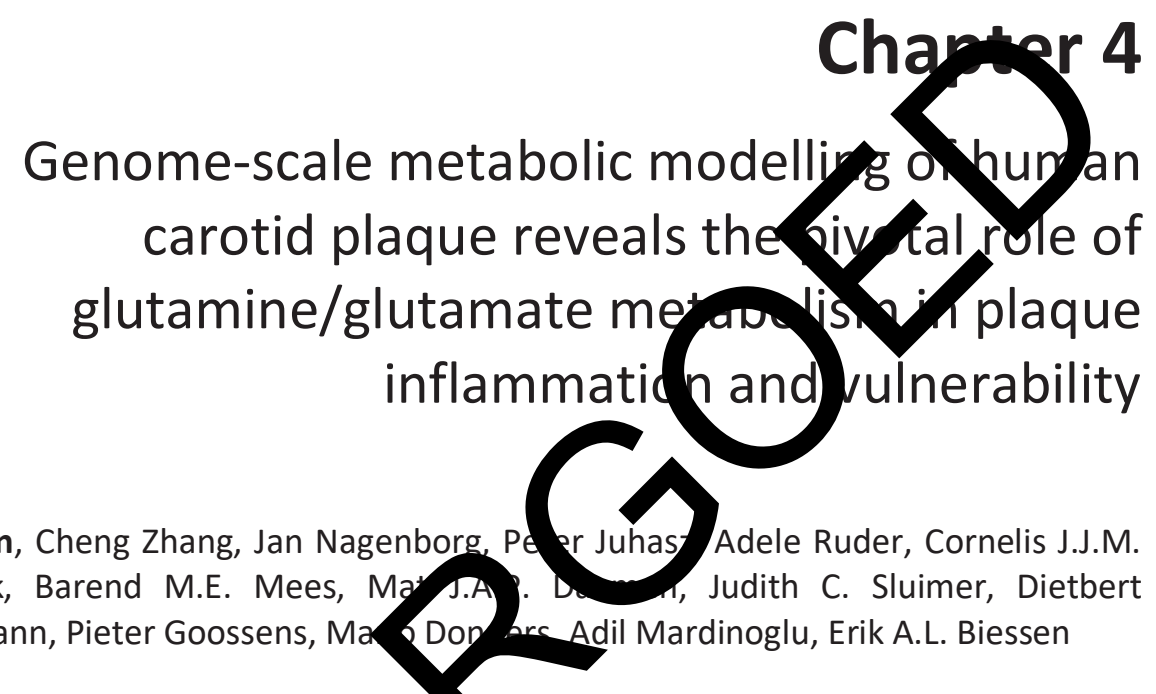

In preparation

Han Jin, Cheng Zhang, Jan Nagenborg, Pe r Juhas Adele Ruder, Cornelis J.J.M. Sikkink, Barend M.E. Mees, Ma J.A . Dumer, Judith C. Sluimet Diert Neumann, Pieter Goossens, Ma Sor hreAdil Mardinoglu, Erik A.L. Biessen

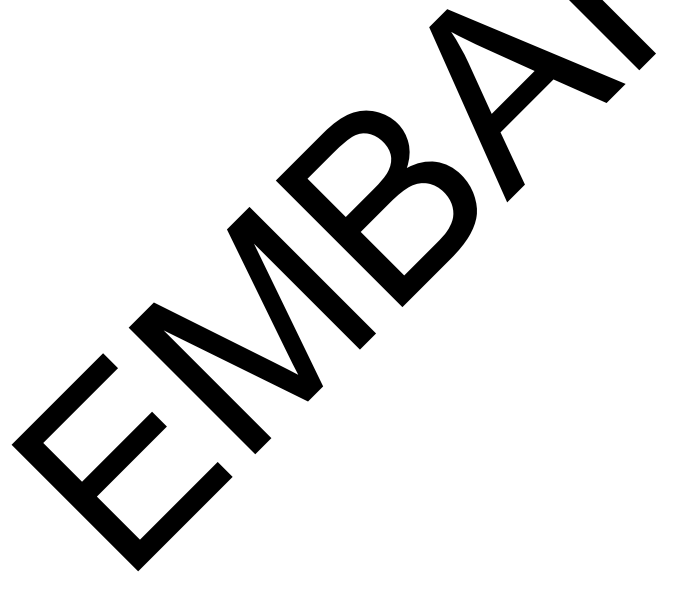




\section{Chapwar 5}

Towards a function-based model of acoph ge plasticity: the Macros ee plavorm

Margaux A.C. Fontaine, Han Jin, Marie Oestreich, Jo m Huckr de, Pieter Goossens, Mat Rousch, Erwin Wijnands, Ludwig Dubg, Tr.PH Naarenburg, Joachim Schultze, Judith C. Sluimer, Thomas Ulas* fieve T nerman*, Erik A.L. Biessen*

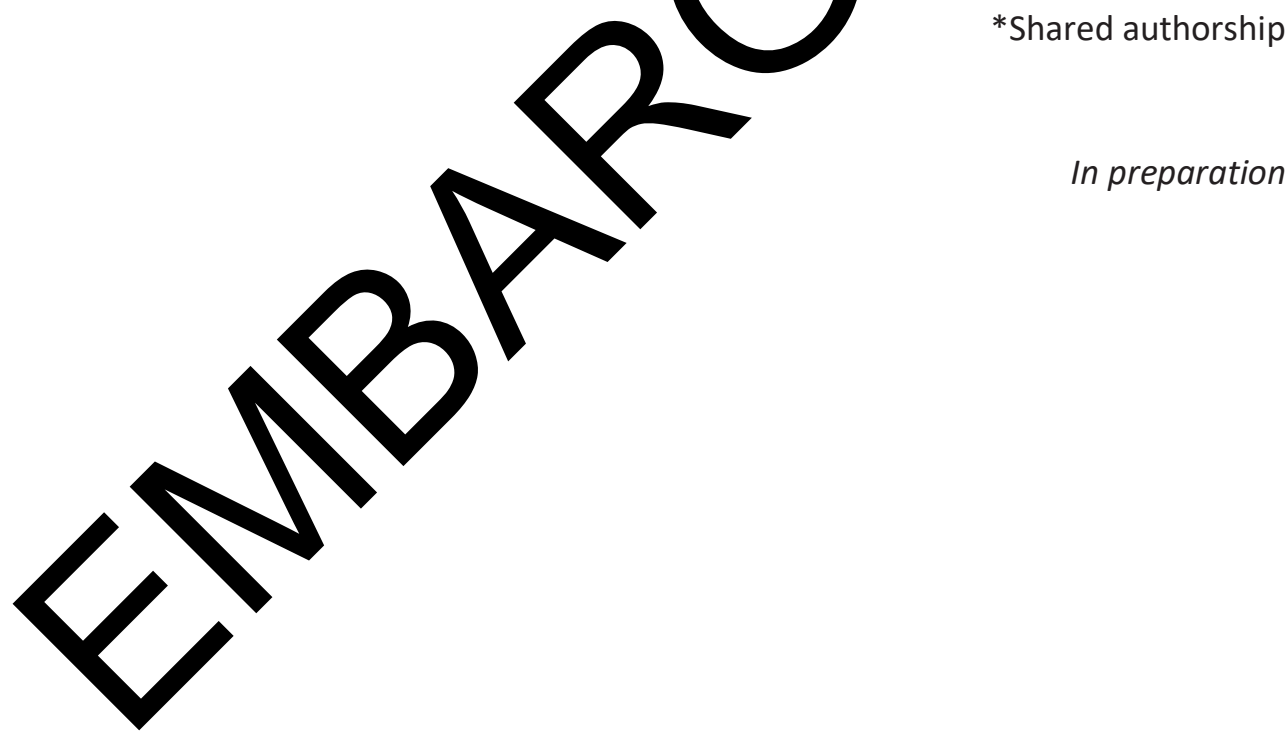




\section{Chapwar 6 Blood milieu in acute myocardial in arct on reprograms human macropha es or trama \\ Margaux A. C. Fontainet, Han Jint, Mick agliard Marnousch, Erwin Wijnands, Monika Stoll, Leon Schurgers, Chris Reute hgsperger Casper Schalkwijk, Nynke M. S. van den Akker, Daniel G.M. Molip ars Jlestad Jan Eritsland, Pavel Hoffman, Mona Skjelland, Geir $\varnothing$. Anderse Pål ukrus,, soél M.H. Karel, Evgueni Smirnov, Bente Halvorsen, Lieve Temmer ont Biessent}

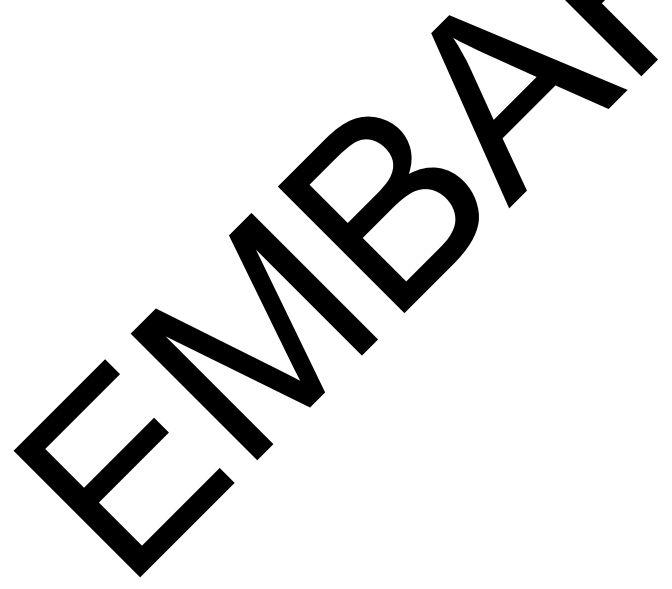

†Authors contributed equally

Submitted 


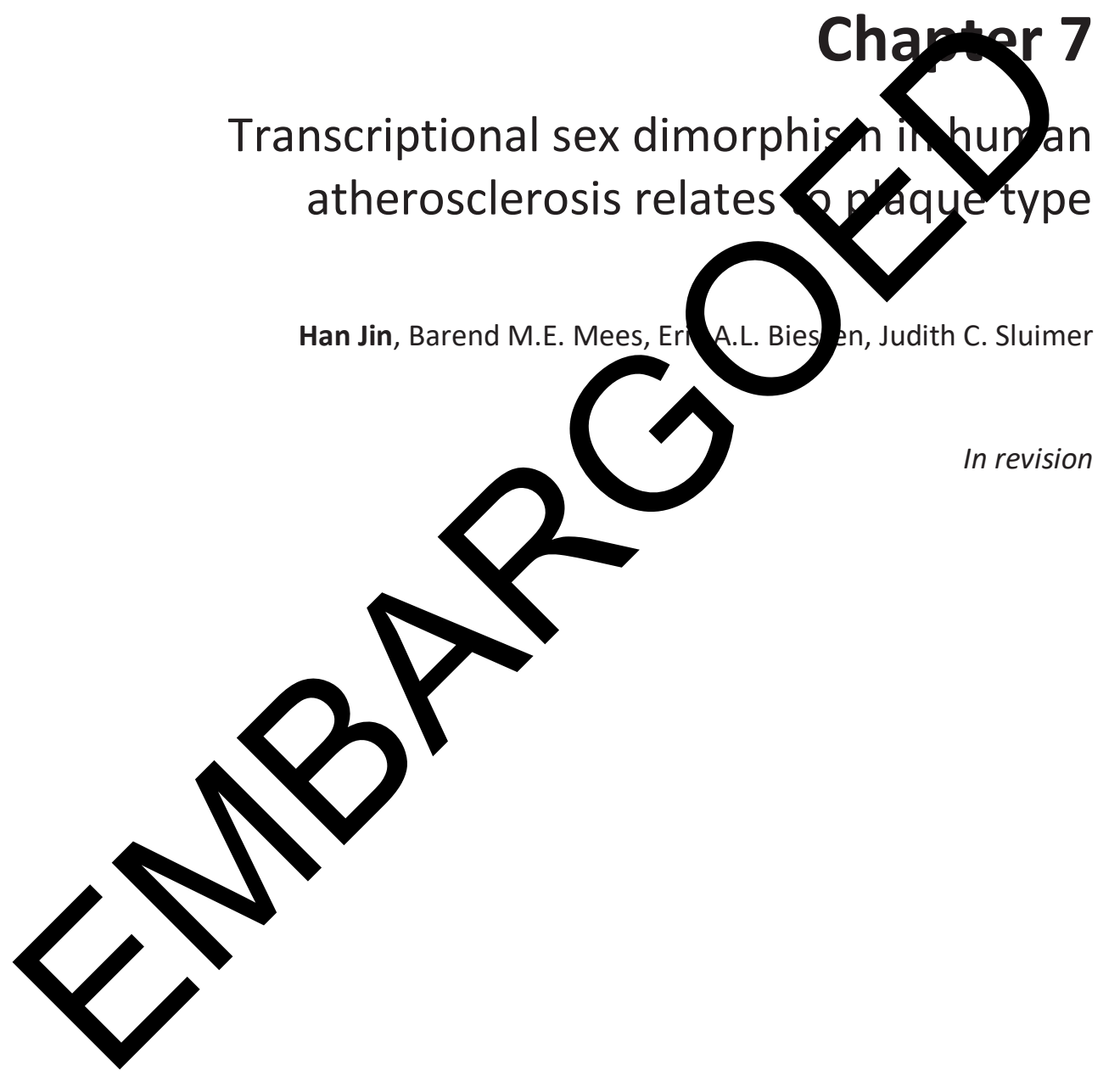




\section{Chapter 8}

General discussion 

Atherosclerosis is a complex disease hallmarked by chronic inflammation and lipid accumulation in the artery wall ${ }^{1}$. Various cell populations and their interplay are involved in disease progression ${ }^{2}$. Despite many efforts that have been made to study atherogenesis, a computational-driven, systematic, and comprehensive disease model incorporating multiple levels of biomolecules and their functions to study atherosclerosis is still lacking. In this thesis, we aimed at deploying state-ofthe-art biotechnology such as high-throughput profiling of genes/proteins/metabolites in combination with computational analysis strategies to dissect the underlying mechanisms in atherosclerosis. Our results demonstrate the power of applying in silico computational analysis for both pathological discovery and risk prediction in cardiovascular research. The main conclusions and discussion points are as follow:

1. This thesis presents several successful applications of machine learning in cardiovascular research for risk prediction and potential therapeutic target identification (chapter 2-6).

2. This thesis highlights the advantages of network-centred analysis compared with traditional bioinformatics approaches for high-throughput omics analysis (chapter 2, 4 and 6).

3. We systematically compared multi- with single-omics analysis in terms of robustness, biological significance and clinical translatability, and illustrated the sensemaking of extracted and integrated domain knowledge from multiple omics data compared with single-omics data (chapter 3 ).

4. This thesis shows the importance of harmonising in silico dry-lab analyses and in vitro wet-lab experiments to obtain solid findings and to reach high reliable conclusions (chapter $\mathbf{3}$ ).

5. Through in silico analyses, we were able to establish several network models depicting the plaque metabolism as well as the mechanisms of plaque stabilization and intraplaque inflammation. Analysing these models, we proposed several promising therapeutic targets and strategies for atherosclerosis intervention (chapter 2, 3, 4 and 6).

In this chapter, I will provide a meta-analysis of the key findings in my thesis, discuss them against current knowledge in the field, and offer future perspectives for further study or application. 


\section{Machine Learning in biomedical research: make things simple or complicated?}

Machine learning approaches were extensively used in this thesis to analyse the high-throughput data generated on plaque and plaque contained inflammatory cells. In general, we applied two machine learning strategies: supervised and unsupervised learning.

Supervised learning aims at assigning a label to an individual sample that for example can be related to a specific category. In this case, label assignment is realized by a classification model trained on labelled data. In biomedical research, supervised learning is of great interest and particularly useful in predicting disease outcomes based on patient's clinical measurements, or establishing the association between molecular changes and clinical syndrome ${ }^{3}$. For instance, in chapter 6 we exploited supervised machine learning models on the functional profiles of human macrophages reprogrammed by AMI patients' serum, and successfully demonstrated the capability of the functional measurements of macrophage behaviours for patient stratification as well as risk prediction. Similarly, in chapter 3 we used an integrative machine learning prediction model to select key elements from multiple omics datasets, and identified a serum response factor (SRF)regulated network associated with smooth muscle and fibroblast-like cells controlling plaque instability.

Machine learning prediction models usually operate as a black box linking data and decisions without providing explanations necessary for decision-making. This may cause a considerable amount of confusion if not concern to biomedical researchers ${ }^{4}$. With the development of more sophisticated models such as deep neural network, the learning and predicting processes become even more abstract and opaque ${ }^{5}$. Despite the successful application of such powerful prediction models in clinical diagnosis ${ }^{6,7}$, there is a need for explainable and interpretable modelling approaches to demystify the black-box model and to better understand the underlying biological mechanisms.

To overcome this issue, in chapter 6, we deployed an explainable machine learning strategy (i.e., Shapley Additive exPlanations, SHAP ${ }^{8}$ ) to reverse-engineer our prediction model based on functional profiles of AMI patients, to answer "why" the model assigns a profile to the AMI rather than control serum exposure group. Shapley values reflect the contribution of each individual feature to the outcome prediction. Compared with feature importance embedded in prediction models such as $\mathrm{LASSO}^{9}$ and tree-based classifiers, which only provide an (often coefficientor frequency-based) numeric weight of a feature's contribution to the prediction, the game theory-based Shapley values helped us to understand to what extent a measurement/feature is pushing the prediction of a sample towards a particular 
phenotype. Based on the Shapley values for the functions in the functional profile, we were able to identify how macrophage behaviour is impacted by the patient's blood circulatory system. Aside from that, in chapter 3, we applied a multi-omics supervised model projecting multiple high-dimensional omics data into a lowdimensional common space for predicting plaque phenotype. This low-dimensional space offered a straightforward visualization of the sample distributions and decisive hyperplane for sample phenotype segregation. We further decomposed the integrative prediction model by analysing component-component relationships and component-feature relationships to discover the most predictive features to plaque phenotype. Those selected features revealed an SRF-driven network associated with atherogenic processes and plaque stability. This chapter demonstrates once again in the context of the machine learning applications in this thesis, that we can offer understandable interpretations and biological meaningful discoveries to biomedical researchers, while utilizing the advantages of supervised machine learning in our research.

Unsupervised learning in the form of categorizing unlabelled data into different meaningful groups, was also extensively used in this thesis. Weighted Gene Coexpression Network Analysis (WGCNA) ${ }^{10}$, an unsupervised network-based clustering analysis was employed to group co-expressed genes into functional gene modules (chapter $\mathbf{2}$ and 6). Most published studies of applying WGCNA on gene expression data do not visualize the co-expression network as a whole, lacking a comprehensive overview of the network structure. However, in chapter 2 we successfully presented the gene co-expression network structure established on human atherosclerotic plaques, and observed the relationships between coexpression modules with a $\mathrm{T}$ cell, interferon, and angiogenesis signature, respectively, which was subsequently demonstrated using Bayesian network inference. In addition, hierarchical agglomerative clustering in combination with heatmap presentation was widely used in this thesis. A typical example can be found in chapter $\mathbf{5}$, where we used hierarchical clustering in conjunction with canonical correlation analysis (CCA)-based multi-omics integration to analyse transcriptional programming and functional behaviour of human macrophage activation. A common feature of these analyses is that, while identifying novel communities based on the similarity of biological entities, they provide straightforward and understandable visualization to researchers.

Our studies show that machine learning and data mining can help researchers to unravel hidden knowledge that is not directly observable from the data. With the rapid development of computer science, the complexity of algorithms and learning models is constantly increasing. However, more and more new techniques have been developed to help researchers to interpret machine learning models. Summarizing all above, we showed that the most widely applied approaches 
applicable in biomedical research have a series of common features, which are interpretable, visually, and biologically meaningful, which could help biomedical researchers to explore data at minimal learning and time cost. 


\section{Network-centred analysis: beyond conventional bioinformatics}

The conventional bioinformatic strategy for high-throughput gene expression analysis aims to identify genes that are differently expressed between conditions, which is an essential step for revealing the dysregulated functions and pathways associated with disease-induced changes. Despite the extensive application of this approach, screening individual biological entities solely based on a differential expression does not capture the relationships between the selected entities. This strategy ignores the knowledge hidden in the omics data, i.e., the co-functions of the selected biological entities, which could be identified and reconstructed by a more advanced approach.

Network-centred approaches in combination with unsupervised clustering overcome this shortcoming. Aiming at constructing a biological network from omics data, these network-centred approaches allow the linkage of different biological entities. The identification of functionally associated clusters through networkbased clustering analysis could provide a more contextualized explanation of the omics data. This is mainly owing to the evaluation of the associations between biological entities, a significant step beyond individual screening from omics data.

A distinctive feature of this network modelling is that the constructed biological networks often exhibit a scale-free property ${ }^{11}$. The scale-free property implicates that the majority of features are connected with or dominated by just a few hubs of high importance ${ }^{12}$. WGCNA's assumption that gene networks are scale-free is a key feature of this approach, when comparing with other clustering approaches for transcriptomics analysis ${ }^{13}$. This criterion was indeed seen to apply for the interactions between biological entities in our reconstructed biological networks (chapter $\mathbf{2}$ and 6), regardless of whether they involved gene co-expression ${ }^{14}$ or in protein-protein interactions ${ }^{15}$. The scale-free property of a co-expression network also implies that hub genes, that have connection numbers much higher than the average, can be considered as major dominators of the whole system. These hubs can be identified through centrality analysis. According to the scale-free property, the loss of the hubs will disconnect massive low-degree nodes, causing the whole (or parts of the) network to collapse ${ }^{15,16}$. Therefore, targeting the hubs of a disease network may destabilize the whole disease network, allowing precise intervention in the disease-induced environment. Taken together, constructing a scale-free biological meaningful network followed by prioritizing network hubs may give opportunities for novel drug target discovery, as it was presented in chapter $\mathbf{2}$.

Biological networks can be non-directional (association) or directional (causality). When describing gene co-expression or protein-protein interactions, a nondirectional network model fits this scenario. However, some biological networks, such as transcription factor-driven gene regulatory networks and metabolic 
networks, are directional. Establishing a biological network with directional connections can therefore help us to understand the causal relationships between biological entities. In chapter 2, we extended the human plaque WGCNA network with Bayesian network inference to identify the causal relationships between the functional co-expression gene clusters, and successfully revealed the effects of $T$ cell functions on interferon-related cytokine production and angiogenesis in atherosclerotic plaque. Another directional network from this thesis is the genomescale metabolic network that we used to reconstruct the whole plaque metabolic system. This is a directional network linking plaque metabolites by metabolic reactions, in which we identified dysregulated glutamine/glutamate pathways that associate with macrophage functions by network centrality analyses. This pathway could serve as a specific target for immunotherapy in the treatment of atherosclerosis.

Having established a biological network, various analyses can be applied to further explore the network's biological capacity. For example, network proximity-based drug repurposing can be applied on an interactome network to screen the best candidate drugs to the pharmacological target ${ }^{17-19}$. In system biology, a network model can represent the associations between biological entities from different omics, creating a biological system that can be used for further exploration. Summarizing all above, this thesis illustrates the advantages of network-centred over conventional gene-centred analytical strategies in terms of systematics, interpretability and contextualization.

Although the network-centred approaches are very powerful, many of the biological networks still rely on differential expression analysis of the network nodes to enable validation or assessment of network functionality. For example, through gene differential expression analysis of the gene co-expression modules, we identified the activation of T cell, interferon and angiogenesis-related pathways in vulnerable plaque (chapter 2). Therefore, differential expression analysis is still a fundamental step in high-throughput omics data analysis and needs to be explored first. Having obtained gene differential expression, it can be integrated with the network model in a more advanced and elegant way, like the one we used to link gene expression with metabolic reactions in the genome-scale metabolic modelling study (chapter 4). 


\section{Multi-omics integration for improved sensemaking vs single-omics analysis}

Analyses of high-throughput omics data have so far led to numerous breakthroughs in biomedical sciences ${ }^{20}$. However, screening key biological entities and/or modelling disease-induced systems from a single layer of omics provides limited information that is only restricted within this omics layer. RNA-seq and microarraybased transcriptional profiling have produced a huge amount of quantitative expression data for more than ten thousand genes/transcripts. However, transcriptomics data do not always reflect the changes that occur at the protein level, due to premature RNA breakdown, post-translational modification and protein degradation, amongst others. Proteomics, as well as metabolomics, are restricted by their limited coverage and relatively incomplete annotation of the biological entities. Thus, mining different omics could identify inter-omics complementary discoveries, which can be subsequently integrated into a comprehensive biological system to provide a systematic view.

In this thesis, we started with an analysis of transcriptomics data, using coexpression network modelling to prioritize the key genes for plaque T cell activity (chapter 2). In chapter $\mathbf{3}$ we integrated the transcriptomics data with proteomics/peptidomics using a machine learning-based multi-omics integration model to predict plaque phenotype and identify key elements that were associated with plaque instability. Based on this model, we compared the advantages of multiomics over single-omics analysis. The fully integrated model provided biologically more meaningful results than the single-omics model, without loss of prediction power, and offered deeper insights into the regulatory pathways in plaque destabilization.

Our multi-omics integration strategy was extended to genome-scale metabolic modelling by integrating transcriptomics with publicly available metabolic databases, as well as metabolomics to study disease-induced metabolic alterations (chapter 4). Through this network-based integrative analysis, we were able to reconstruct the human plaque metabolic system as a metabolic network, in which the metabolic reactions were connected with transcriptomics. Having integrated transcriptomics data into the genome-scale metabolic network, we were able to extract the expression of the corresponding enzymes regulating the reactions, thus inferring the metabolic activities of the whole network. In this context, we like to note that given multiple omics data, screening biological entities based on differential expression from every omics data can identify disease-associated biomarkers. However, this approach cannot provide further insights on the interrelation between biomarkers identified from different omics layers, and thus lack a comprehensive view of the whole system. In contrast, our study used a more 
advanced approach to utilize the full information from transcriptomics and metabolomics as well as publicly available databases to reconstruct the plaque metabolic system, simultaneously providing a high-resolution view of the system at a sub-cellular level.

In addition, to study macrophage functional polarization and reprogramming, we integrated transcriptomics with functionomics data. The latter involved a comprehensive evaluation of 19 macrophage functionalities (chapter 5). The consistency between transcriptomics and functionomics profiles of macrophage responses was further explored in chapter 5, through a canonical correlation analysis (CCA)-based approach to simultaneously extract the highly correlated information from the two omics domains. Using this approach, transcriptional and functional profiles of polarized macrophages by 28 stimuli plus control were projected into a common low-dimensional space, after which hierarchical clustering was used to group macrophage polarization based on the information shared between the two different omics layers. This integrated clustering resulted in a distinct grouping of stimulated macrophages compared with using only either transcriptomics or functionomics, showing the validity of this conjunctional approach in generating a consensus model, supported by both omics layers. Moreover, having projected transcriptomics and functionomics to the common space, macrophage functions were further clustered according to the functiongene associations in this common space. Compared with our integrative analysis, clustering on functionomics-only was limited by the information provided by the 19 available functional assays, whereas results of clustering of the high-dimensional transcriptomics were not directly translatable to functional activity. This integration also allows the identification of genes associated with a certain function, and even holds potential in predicting macrophage functions from the transcriptional profile through CCA-based transfer learning. With the above characteristics, this functiongene integrative platform can be used as a swift and low-budget platform for toxicogenomics analysis and in silico drug screening.

In chapter 6, the individual functional measurements were linked with the corresponding transcriptional profiles by correlating functional measurements with the gene co-expression modules analysed by WGCNA on transcriptomics. Surprisingly, the overall module correlations showed high consistency with the importance of functional measurements for the prediction of patient phenotype, implicating the shared information between transcriptomics and functionomics for risk stratification. By investigating correlations between macrophage functions and macrophage gene functional co-expression clusters, we successfully identified the correspondence between functional measurements and gene expression as well as the associated pathways, facilitating the understanding of the underlying biological processes in association with macrophage functional behaviours. 
Summarizing above, omics integration analyses presented in this thesis showed the merits of integrating transcriptomics data, a relatively easily approachable and well-annotated omics layer, with omics data of a higher level, i.e., from proteomics to metabolomics. This was extended to the functional domain, linking transcriptomics to functionomics data, providing unprecedented insights into the regulation of functions. Integration has helped to overcome the intrinsic limitations of the individual data layers (proteome: low coverage, with a bias towards abundant genes; genome: very high dimensionality, lack of translatability; metabolome: interpretation issues; functionome: limited resolution), and benefit translatability and interoperability of the ensuing model.

Multi-omics integration pursued in this thesis involved different strategies and led to 1) discovery of an SRF-driven gene-protein network controlling plaque stability; 2) establishment of a genome-scale metabolic atlas for human carotid plaque; and 3) dissection of circulatory macrophage functional changes upon exposure to the blood environment in $\mathrm{AMI}$, and their relation to post-MI repair. These multivariate, systematic discoveries could not be achieved either by single omics analysis or by conventional biomedical experimentation. Incorporating additional omics layers, such as genomics and lipidomics, may further strengthen the models and provide deeper insights into the disease mechanisms. In future, extended biological experiments would need to validate these findings and translate the discoveries into clinical application.

In conclusion, this thesis presents several clear illustrations of the potential of integrating omics domains, using study object guided strategies, over single-omics strategies for biomedical research. 


\section{In silico vs in vitro: guiding each other towards a promising future}

With the development of high-throughput sequencing technologies and machine learning/data mining algorithms, in silico analyses have been increasingly impacting traditional biomedical research and playing a more and more important role. Compared with traditional biomedical analytical strategies, which mostly focus on only one factor to the disease, high-throughput in silico analysis usually starts with a huge number of biological entities, such as genes, proteins, metabolites, and drugs, through an extensive exploration of the omics data by statistical models and computer-aided simulation procedures to grasp the global changes at molecular levels and thus identify the potential hits and drug targets. With this characteristic, high-throughput in silico analysis can be carried out in a hypothesis-free manner without biasing to a pre-established assumption, providing unprecedented implications and directions for further exploration.

More and more publicly available databases for high-throughput data, such as Gene Expression Omnibus (GEO), as well as the biological pathway databases such as $\mathrm{KEGG}^{21}$ and WikiPathways ${ }^{22}$, have been established and are continuously expanding, facilitating the data sharing and cross-validation between research groups. Biological big-data mining from publicly available databases becomes a promising research direction. Compared with traditional wet-lab experiments, computer algorithms are well-known for their flexibility, reproducibility, and are less timeand finance-consuming. Based on these characteristics, in silico analysis can be executed by iteratively adjusting and optimizing parameters to proximate the best performance of an algorithm, which is far more flexible and time-efficient to the experimental setup and parameter adjustment in wet-lab experiments.

However, for both dry-lab and wet-lab experiments, one relies on the quality of experimentation of the other to reach high reliable conclusions. While most wetlab experiments need to be done in-house, researchers often do not have access to all relevant accessory data. Moreover, pathway- and interaction-based biological databases are often built without weighing for quality of experimental evidence or equalizing scores of different backgrounds or formats (e.g., direct experimental evidence vs text mining, where the proximity of terms is used as a criterion). Furthermore, merging of datasets of different sources requires alignment, batch correction, or bridging steps at the minimum; nevertheless, the differences in experimental settings cannot be completely corrected.

Therefore, results from in silico analysis need to be validated by biological in vitro experiments and eventually in vivo to verify the validity and reliability of the findings. Without this critical step, in silico findings are only confined within the simulation environment, lacking the translational perspective from (pre)clinical experimentation. In addition, in silico analysis also have its limitation, namely that 
the findings could be biased towards prior knowledge discovered from previous biological experiments when using established biological databases. That is why, further in vitro experiments not only can validate the in silico results, but also can enrich and extend the results in a biological context.

Interestingly, while computer-aided and computational-based approaches have significantly accelerated the knowledge discovery in biomedical research, the development of new learning algorithms also benefits from biological knowledge. For example, Artificial Neural Networks (ANN) ${ }^{23}$ and their extensions such as Deep Neural Networks (DNN), are machine learning models consisting of blocks representing neurons, synapses and signal transduction originating from the biological neural system ${ }^{24}$. Genetic algorithms are another type of nature-inspired algorithms that are designed for flexible search by simulating biological evolutional and natural selection processes including reproduction, mutation, recombination, and selection to achieve optimised results ${ }^{25}$.

While both in vitro and in silico analyses can guide biomedical research to a farreaching development, in chapter $\mathbf{3}$, we harmonised these two complementary analyses by following the biological experiment-data analysis-biological validation procedure. This procedure showed the complementarity of each other, and a promising and challenging perspective for interdisciplinary collaboration. Furthermore, anticipated in vitro, ex vivo and in vivo biological experiments have been planned to validate and extend the results in chapter $\mathbf{2}$ and $\mathbf{4}$. It is believed that further in vitro study will significantly complement and improve the drug targets identified through in silico analyses in chapter 2, 4 and 6. 


\section{Therapeutic targets for plaque stabilization and regression}

In this thesis, we proposed several therapeutic targets for plaque stabilization. In chapter 2, through drug screening and repurposing based on connectivity score between network-organized gene module expression and drug perturbed gene expression, we discovered EGFR inhibitors as drug candidates targeting the T cell activities and angiogenesis for the interventional therapy for plaque stability. Another therapeutic target was provided in chapter 3, i.e., the dysregulated SRF regulating SMCs functions and potentially controlling plaque stability, which was identified through an integrated analysis of transcriptomics and proteomics/peptidomics using a multi-omics machine learning model. Moreover, in chapter 4, we reconstructed a plaque-specific genome-scale metabolic network followed by network topological analysis, and identified the glutamate/glutamine pathways as a potential target for immunomodulating plaque microenvironment so as to interfere with plaque stability.

Current therapeutic strategies for atherosclerosis often rely on plasma lipid lowering, increasingly even in "normolipidemics" subjects. For example, cholesterol medications such as statins, and new generation drugs including PCSK9 inhibitors, can significantly reduce the low-density lipoprotein cholesterol (LDL-C) and slow down lipid accumulation and build-up of a lipid core in the arterial wall. In parallel, the control of other comorbidities of cardiovascular diseases are targeted, such as hypertension, type 2 diabetes and morbid obesity, as well as "modifiable" cardiovascular risk factors including smoking, diet and exercise, to reduce the risk of cardiovascular events. Despite that great efforts have been made to control the risk of acute events induced by atherosclerosis, not all patients tolerate or are responsive to these therapeutic interventions. Thus, a novel target that can fundamentally slow down and regress the plaque progression is still lacking, requiring a more advanced and targeted therapeutic strategy for atherosclerosis intervention.

The discovered therapeutic targets in our studies can be roughly divided into two types: targeting key factors in SMCs to modulate SMCs functions to stabilize the plaque content, or modulating the immune microenvironment to reduce inflammatory responses so that to decelerate or reverse the plaque progression. Several trial studies have been carried out to control the risk of atherosclerosisinduced cardiovascular events through the reduction of inflammation in plaque atheroma by anti-inflammatory drugs. For example, the CANTOS trial used Canakinumab, a monoclonal antibody targeting interleukin-1 $\beta$ to reduce vascular inflammation so as to prevent myocardial infarction. This clinical trial showed a significantly lower rate of recurrent cardiovascular events than placebo under a particular dose without lowing the plasma lipid levels ${ }^{26,27}$. 
Our studies proposed several immune-related therapeutic targets, underpinned the promising perspective of immunomodulation in atherosclerosis therapy. Indeed, key pathways involved in atherosclerosis, such as immune response and angiogenesis, were also found to have an important role in tumour and cancer development. Nowadays, a lot of public research resources and analytical tools focusing on cancer, tumour and immune-associated research (e.g., The Cancer Genome Atlas, TCGA, and in silico immune infiltration analysis) have been established, facilitating the study of immune processes involved in atherosclerosis. With the massive efforts and resources that have been put into cancer research, atherosclerotic research can be beneficial from cancer/tumour research, especially in understanding the immune response of and discovering immunomodulatory therapy for atherosclerosis.

The complex cell populations and pathways involved in the progression of atherosclerosis make the development of effective drugs and targets for such a complex disease very challenging. Meanwhile, this gives researchers opportunities to design novel therapies for atherosclerosis that target other processes not affected by current drugs. This thesis demonstrates the advantages of analysing high-throughput data using state-of-the-art approaches to study the mechanisms of atherogenesis as well as to discover novel therapeutic targets for atherosclerosis. With the combination of machine learning, network analysis and omics integration analyses on high-throughput data, it is foreseeable that more effective and targeted therapeutic targets and strategies for atherosclerosis will be developed and applied in future. 


\section{Concluding remarks and future perspective}

In this thesis, we performed various computational analyses, including machine learning clustering and classification, network modelling and multi-omics integration on high-throughput omics data to study mechanisms of atherosclerosis and discover potential drug targets for disease therapy. These bioinformatic and computational approaches appeared to be robust, flexible and reproducible in biomedical data analysis, providing insights that could not be obtained from traditional experimental methods. Thus, this thesis illustrates the promising perspective and we expect that more and more applications of high-throughput omics data and computational analysis in atherosclerosis research will follow soon.

High-throughput biomedical research is entering a new era, i.e., the single-cell sequencing technology, enabling the evaluation of gene expression at a single-cell level. Considering the complex and heterogeneous cell composition of the atherosclerotic lesion, single-cell sequencing is particularly suitable and useful for the discovery of novel cell subpopulation (e.g., heterogeneous macrophage populations), the differentiation and development of plaque cell lineages, and the cell-cell interactions (e.g., plaque $\mathrm{T}$ cells and macrophages) in atherosclerosis research. Even though we were able to deconvolute our microarray data of bulk carotid endarterectomy tissue into several major immune cell populations (chapter 2), this however needs to be validated by single-cell sorted data. Moreover, existing approaches for bulk microarray and RNA-seq data, such as gene set enrichment analysis, transcriptional regulatory analysis, as well as metabolic network modelling can be adapted to single-cell sequencing data to discover new findings within a homogeneous cell population, rather than a mix of heterogeneous cell populations, in atherosclerotic plaque. With numerous successful applications of single-cell techniques in atherosclerosis in the past 2-3 years, further breakthroughs are to be expected in the near future.

In view of the expansion of high-throughput biological data and the development of computing power and algorithms, biomedical research has to embrace Big Data. Big Data allow us to model the biological system more precisely and elaborately using intelligent models, of which the complexity and performance are far beyond our imagination. It will help humanity to understand biology and support clinical decisions. Just like autonomous driving, many efforts remain to be done for in silico medicine, but the day will inevitably come that we all will not only accept but also adopt Al-based precision medicine. 


\section{REFERENCES}

1. Libby P, Ridker PM, Hansson GK. Progress and challenges in translating the biology of atherosclerosis. Nature. 2011;473(7347):317-325.

2. Orekhov AN, Andreeva ER, Andrianova I V., Bobryshev Y V. Peculiarities of cell composition and cell proliferation in different type atherosclerotic lesions in carotid and coronary arteries. Atherosclerosis. 2010;212(2):436443.

3. Sidey-Gibbons JAM, Sidey-Gibbons CJ. Machine learning in medicine: a practical introduction. BMC Med Res Methodol. 2019;19(1):64.

4. Murdoch WJ, Singh C, Kumbier K, Abbasi-Asl R, Yu B. Definitions, methods, and applications in interpretable machine learning. Proc Natl Acad Sci U S A. 2019;116(44):22071-22080.

5. Liu W, Wang Z, Liu X, Zeng N, Liu Y, Alsaadi FE. A survey of deep neural network architectures and their applications. Neurocomputing. 2017;234:11-26.

6. Hannun AY, Rajpurkar P, Haghpanahi M, et al. Cardiologist-level arrhythmia detection and classification in ambulatory electrocardiograms using a deep neural network. Nat Med. 2019;25(1):65-69.

7. Gurovich $Y$, Hanani Y, Bar O, et al. Identifying facial phenotypes of genetic disorders using deep learning. Nat Med. 2019;25(1):60-64.

8. Lundberg SM, Allen PG, Lee S-I. A Unified Approach to Interpreting Model Predictions.

9. Tibshirani R. Regression Shrinkage and Selection Via the Lasso. J R Stat Soc Ser B. 1996;58(1):267-288.

10. Langfelder $\mathrm{P}$, Horvath S. WGCNA: An R package for weighted correlation network analysis. BMC Bioinformatics. 2008;9(1):1-13.

11. Broido AD, Clauset A. Scale-free networks are rare. Nat Commun. 2019;10(1):1-10.

12. Barabási A-L, Gulbahce N, Loscalzo J. Network medicine: a network-based approach to human disease. Nat Rev Genet. 2011;12(1):56-68.

13. Zhang B, Horvath S. A general framework for weighted gene co-expression network analysis. Stat Appl Genet Mol Biol. 2005;4(1).

14. Barabási A-L, Oltvai ZN. Network biology: understanding the cell's functional organization. Nat Rev Genet. 2004;5(2):101-113.

15. Jeong $\mathrm{H}$, Mason SP, Barabási AL, Oltvai ZN. Lethality and centrality in protein networks. Nature. 2001;411(6833):41-42.

16. Albert $\mathrm{R}$, Jeong $\mathrm{H}$, Barabási AL. Error and attack tolerance of complex networks. Nature. 2000;406(6794):378-382.

17. Guney E, Menche J, Vidal M, Barábasi AL. Network-based in silico drug efficacy screening. Nat Commun. 2016;7(1):1-13.

18. Lotfi Shahreza M, Ghadiri N, Mousavi SR, Varshosaz J, Green JR. A review of 
network-based approaches to drug repositioning. Brief Bioinform. 2018;19(5):878-892.

19. Cheng F, Desai RJ, Handy DE, et al. Network-based approach to prediction and population-based validation of in silico drug repurposing. Nat Commun. 2018;9(1).

20. Costa FF. Big data in biomedicine. Drug Discov Today. 2014;19(4):433-440.

21. Okuda S, Yamada T, Hamajima M, et al. KEGG Atlas mapping for global analysis of metabolic pathways. Nucleic Acids Res. 2008;36(Web Server issue):423-426.

22. Martens $M$, Ammar A, Riutta $A$, et al. WikiPathways: connecting communities. Nucleic Acids Res. 2021;49(D1):D613-D621.

23. Jain AK, Mao J, Mohiuddin KM. Artificial neural networks: A tutorial. Computer (Long Beach Calif). 1996;29(3):31-44.

24. Lecun Y, Bengio Y, Hinton G. Deep learning. Nature. 2015;521(7553):436444.

25. Whitley D. A genetic algorithm tutorial. Stat Comput. 1994;4(2):65-85.

26. Ridker PM, Everett BM, Thuren T, et al. Antiinflammatory Therapy with Canakinumab for Atherosclerotic Disease. N Eng/ J Med. 2017;377(12):11191131.

27. Ridker PM, Thuren T, Zalewski A, Libby P. Interleukin-1 $\beta$ inhibition and the prevention of recurrent cardiovascular events: Rationale and Design of the Canakinumab Anti-inflammatory Thrombosis Outcomes Study (CANTOS). Am Heart J. 2011;162(4):597-605. 




\section{Chapter 9}

Summary | Samenvatting | 总结 



\section{Summary}

Cardiovascular diseases are one of the leading causes of death worldwide. The main underlying cause of cardiovascular diseases - atherosclerosis, is a chronic inflammatory disease, that implicates several complex biological processes, including lipid accumulation, inflammatory responses and fibrosis, in mutual interaction. To date, despite significant efforts that have been made towards a better understanding of atherogenesis, systematic and comprehensive modelling of atherosclerosis is still lacking. In this thesis, we deployed machine learning and computational-based data-driven methods on biological omics big-data to construct complex atherosclerotic systems and to clarify the disease mechanisms systematically. Moreover, through in silico analyses and modelling of cardiovascular diseases, we identified several new therapeutic targets for clinical intervention.

This thesis mostly focuses on carotid atherosclerosis, which is the main cause of ischemic stroke. In chapter $\mathbf{2}$, based on the transcriptional profiling of human carotid plaques, we constructed a gene co-expression network to unravel the major biological processes and pathways involved in the disease progression. The relationships between the associated pathways were subsequently described by a Bayesian inference network, highlighting the central role of T cell activity in human plaque. Furthermore, the scRNA-seq data exploration and construction of the gene regulatory network underpinned the important function of cytotoxic $\mathrm{CD} 8^{+} T$ cells in plaque destabilization. Finally, through in silico screening of drugs in a publicly available drug database, we identified several epidermal growth factor receptor inhibitors (EGFRi) as top candidates to target T cell-associated gene program in haemorrhaged human plaque, which could guide the design of in vitro and in vivo experiments to test the suitability of EGFRi for the treatment of clinical atherosclerosis.

In chapter 3, we deployed a multi-omics machine learning prediction model integrating transcriptomics with proteomics/peptidomics to stratify high-from lowrisk plaques. We compared the multi-omics with single-omics based models, and illustrated the advantages of truly integrative omics analysis in terms of accuracy, visibility and model interpretability. Our integrative multi-omics model revealed an SRF-driven gene/protein network associated with smooth muscle functions, controlling plaque instability, which could guide efforts for designing novel therapeutic strategies for plaque stabilization.

In addition, by integrating transcriptomics, proteomics, metabolomics and publicly available pathway databases, in chapter 4 we reconstructed a genome-scale metabolic network for human plaque. Based on our knowledge, this is so far the most comprehensive metabolic atlas of human atherosclerotic plaque, providing 
great details of plaque metabolism. Analysing this metabolic network for networkbased topological centrality, we were able to pinpoint the pivotal role of macrophage glutamate/glutamine metabolism in plaque inflammation and vulnerability. This pathway could be a promising immunomodulation target for the prevention and treatment of atherosclerosis.

In chapter 5, we established a high-throughput platform to evaluate the functional changes of macrophages in the context of cardiovascular inflammation. This "functionomics" compendium of human macrophage in response to a variety of stimuli, was subsequently integrated with macrophage transcriptomics, showing macrophage plasticity at both functional and transcriptional levels. This compendium can serve as a reference for the future study of macrophage heterogeneity.

Using this MacroScreen platform, in chapter 6 we evaluated the macrophage functional reprogramming by the systemic environment after acute myocardial infarction. In this chapter, machine learning models were deployed to stratify/distinguish the impact of AMI patients' serum from control on macrophage function. We then integrated functionomics with network-organised transcriptomics of AMI serum exposed cells, and identified gene networks correlating with poor clinical prognosis. Finally, through in silico drug screening, we identified PGE2 signalling as a potential target for the prevention of adverse postinfarct remodelling.

In chapter 7, we showed that sex-specific differences in plaque phenotype contribute considerably to the recently reported transcriptional sex dimorphism of human atherosclerotic plaque. This information is key for future studies of the cardiovascular community to design sex-specific therapeutics for precision medicine.

Finally, in chapter $\mathbf{8}$ we discussed the main findings and the potential perspectives of this thesis, as well as several conceptual questions for the application of in silico analyses in biomedical research.

In conclusion, by applying computational-based analyses, including statistical inference, machine learning, omics integration and network modelling on multiple omics data of human atherosclerosis and blood, we were able to model and analyse cardiovascular diseases at a molecular level, in a systematic manner. These computational and data-driven analyses provided novel insights into the disease mechanisms as well as into therapeutic targets and drugs for the treatment of atherosclerosis. 


\section{Samenvatting}

Hart- en vaatziekten betreffen wereldwijd een van de belangrijkste doodsoorzaken. De belangrijkste onderliggende oorzaak van hart- en vaatziekten, aderverkalking of te wel atherosclerose, is een chronische ontstekingsziekte van middelgrote en grote slagaders, waarin verschillende complexe biologische processen, zoals vetopstapeling, bloedvatvorming, ontstekingsreacties en fibrose, betrokken zijn. Ondanks een sterk toegenomen begrip van dit ziektebeeld, ontbreekt het tot op heden aan een systematische analyse van de moleculaire processen en hun onderlinge samenhang die hieraan ten grondslag liggen. In dit proefschrift hebben we kunstmatige intelligentie ("machine-learning") en computationele datagedreven methodieken toegepast op expressieprofielen van de verkalkte vaatwand maar ook in cultuur gebrachte ontstekingscellen toegepast om het ziekteproces te modelleren. Ook hebben wij, door middel van in silico analyses en modellering van hart- en vaatziekten, verschillende nieuwe therapeutische markers geïdentificeerd voor klinische interventie.

Dit proefschrift richt zich vooral op aderverkalking ("plaque") in de halsslagader, dat als gevolg van ruptuur kan leiden tot een beroerte.

In hoofdstuk $\mathbf{2}$ hebben wij op basis van transcriptionele profilering van menselijke halsslagader endarteriëctomieën een gen co-expressie netwerk geconstrueerd om de belangrijkste biologische processen en paden van de ziekteprogressie te ontrafelen. De relatie tussen "biochemische routes" worden vervolgens beschreven via een Bayesiaans inferentienetwerk, waaruit een centrale rol van Tlymfocyt activiteit in de menselijke plaque naar voren kwam. Bovendien wezen zowel de expressiedata als het afgeleide regulatoire netwerk op een belangrijke rol van cytotoxische $\mathrm{CD} 8^{+}$lymfocyten in de destabilisatie van de plaque. Tenslotte hebben we door middel van computergestuurde screening van geneesmiddelen uit een publiek beschikbaar databestand, epidermale groeifactor receptor remmer (EGFRi) geïdentificeerd als top-kandidaat voor interferentie met dit lymfocytgeassocieerde netwerk in atherosclerose. Dit is een veelbelovend startpunt voor studies in proefdiermodellen naar de effectiviteit van EGFRi voor behandeling van klinische atherosclerose.

In hoofdstuk 3 hebben we een voorspellend model ontwikkeld dat genexpressie profielen integreert met eiwit en peptideprofielen om hoog-risico plaques te kunnen onderscheiden van plaques met een laag risico. We vergeleken daarbij een model verkregen na integratie van deze datalagen met een model afgeleid van enkel genexpressieprofielen, en hebben de voordelen van eerstgenoemde in kaart gebracht (nauwkeurigheid, zichtbaarheid en interpreteerbaarheid). Uit ons integratieve model hebben we vervolgens een serum response factor-gedreven gen/eiwit netwerk geëxtraheerd. Dit netwerk correleerde met de aanwezigheid en 
activiteit van gladde spierfuncties, die belangrijk zijn voor regulatie van de plaquestabiliteit. Deze studie zou op termijn kunnen leiden tot het ontwerp van nieuwe therapeutische strategieën voor plaquestabilisatie.

Door de integratie van gen-, eiwit-, en metabolietprofielen van atherosclerotische plaque en combinatie met in de publieke ruimte beschikbare databestanden, hebben we in hoofdstuk 4 een metabool netwerk voor menselijke plaque gereconstrueerd. Dit is, voor zover ons bekend, de meest uitgebreide metabole atlas van de humane atherosclerotische plaque, en laat een gedetailleerd beeld zien van het metabolisme in de plaque en haar relatie met de klinische prognose van atherosclerose. Uit een diepere analyse van dit metabole netwerk bleek een centrale rol van het glutamaat/glutamine metabolisme van macrofagen in plaque ontsteking en stabiliteit. Deze metabole route zou daarmee een veelbelovend doel kunnen zijn voor preventie en behandeling van atherosclerose.

In hoofdstuk 5 hebben we een testplatform opgezet om de functionele veranderingen van macrofagen onder condities van hart- en vaatziekten te analyseren (de MacroScreen). "Functionomica" analyse middels dit platform resulteerde in een functioneel compendium van de reactie van humane ontstekingscellen (macrofagen) op een verscheidenheid aan stimulantia. Dit compendium werd vervolgens geïntegreerd met de corresponderende genexpressiedata van deze macrofagen blootgesteld aan dezelfde set van stimulantia. Hieruit bleek eens te meer de plasticiteit op zowel functioneel als expressie niveau van dit celtype. Dit compendium kan dienen als referentie voor de toekomstige studie van macrofaag heterogeniteit en voor screeningsdoeleinden

Met behulp van het MacroScreen platform hebben we vervolgens, in hoofdstuk 6, de functionele herprogrammering van macrofagen na blootstelling aan bloed van een patiënt met een acuut myocardinfarct bestudeerd. Ook hier werden machine learning modellen opgebouwd en toegepast om de impact van serum van patiënten met een hartinfarct op macrofaagfunctie te bepalen. Daaropvolgende integratie van de functionele en netwerk-georganiseerde genexpressiesignatuur van AMI serum blootgestelde cellen, leidde tot de identificatie van een genetisch netwerk dat correleerde met een ongunstige klinische prognose van deze patiënt. Tenslotte hebben we, door middel van computergestuurde geneesmiddel screening, een geneesmiddel geïdentificeerd dat dit netwerk mogelijk kan dempen, en dat dus mogelijk ingezet zou kunnen worden voor de preventie van ongunstige prognose na een hartinfarct.

In hoofdstuk 7 hebben we laten zien dat sekse-specifieke verschillen in plaque morfologie in belangrijke mate bijdragen aan de recent gerapporteerde sekse gedreven genetische dimorfisme van humane atherosclerotische plaque. Deze informatie is essentieel voor toekomstige studies van de cardiovasculaire 
gemeenschap om geslacht specifieke therapieën te ontwerpen voor precisie geneeskunde.

Tenslotte bespraken we in hoofdstuk 8 de belangrijkste bevindingen en de mogelijke perspectieven van dit proefschrift, evenals een aantal conceptuele vragen voor de toepassing van in silico analyses in biomedisch onderzoek.

Samenvattend laten we in dit proefschrift zien dat toepassing van computergestuurde analyses, waaronder statistische inferentie, kunstmatige intelligentie, data integratie en netwerktheorie op uitgebreide en complex expressiedatasets van de menselijke plaque en ontstekingscellen, ons in staat stelt om hart- en vaatziekten, op een systematische wijze, op moleculair niveau te modelleren en analyseren. Deze computationele en data-gedreven analyses leverden niet alleen nieuwe inzichten op in de ziektemechanismen maar leidde tevens tot nieuwe therapeutische doelwitten en geneesmiddelen voor behandeling van atherosclerose. 



\section{总结}

心血管疾病是世界范围内人类的头号死因。引起心血管疾病主要原因一一动脉粥样 硬化, 是一种慢性炎症性疾病。它的发生和发展牵涉到几个复杂的生物过程, 包括 脂质积累、炎症反应和纤维化等。这些生物过程之间相互影响和作用, 共同推动着 疾病的发展。尽管研究人员为更好地了解动脉濑样硬化疾病的机理做出了不解努力, 然而, 迄今为止, 我们仍然缺乏一个系统和全面的分子层面上的动脉粥样硬化模型 来描绘该疾病的发展机制。在这篇论文中, 我们将机器学习和基于计算和数据驱动 的研究方法应用于生物组学大数据, 进而构建复杂的动脉码样硬化系统模型用于系 统地阐明疾病机理。此外, 通过对心血管疾病的计算分析和建模, 我们还发现了几 个新的临床干预治疗靶点。

这篇论文主要关注颈动脉粥样硬化，它的发生发展是导致缺血性中风的主要原因。 在第二章中, 基于人类颈动脉斑块的转录组分析, 我们构建了一个基因共表达网络, 以揭示参与疾病进展的主要生物过程和途径。随后, 通过贝叶斯推理网络分析, 我 们刻画了相关生物过程之间的关系, 并凸显了 T细胞活动在人类动脉张样硬化斑块中 的核心作用。此外, 基于单细胞转录组的数据分析和基因调控网络的构建揭示了细 胞毒性 $C D 8^{+} \mathrm{T}$ 细胞在斑块不稳定中的重要功能。最后, 通过对公开的药物数据库中 的药物进行计算篮选，我们确定了几种表皮生长因子受体抑制剂（EGFRi）作为针对 出血性人类斑块中 $\mathrm{T}$ 细胞相关基因进行干预治疗的候选靶向药物。这项发现可以指导 后续体外和体内实验的设计, 以测试 EGFRi 是否适用于动脉硬化的临床治疗。

在第三章中, 我们整合了转录组、蛋白质组和肽组数据并构建了一个多组学机器学 习预测模型, 从而实现对高风险和低风险斑块表型进行预测。我们将多组学模型与 基于单一组学的机器学习模型进行了综合比较, 并详细论述了多组学分析在预测准 确性、结果可视化和模型可解释性方面的优势。通过解构该多组学模型, 我们构建 了一个与血管平滑肌功能相关的由血清应答因子 (SRF) 调控的基因/蛋白质网络, 其在影响玟块不稳定性方面发挥着重要的作用。该发现可用于构建动脉粥样硬化的 全新治疗方案, 进而达到稳定动脉唹样硬化斑并预防斑块破裂的目的。

此外, 通过整合转录组学、蛋白质组学、代谢组学和公开的生物通路数据库, 我们 在第四章中重建了人类斑块的基因层面的代谢网络。据我们所知, 这是迄今为止最 全面的人类动脉㛏样硬化斑块的代谢图谱，提供着斑块代谢的丰富细节。通过分析 这个代谢网络内部结点的拓扑中心性, 我们能够锁定巨噬细胞中谷氨酸/谷氨酰胺代 谢通路在斑块炎症和脆弱性中扮演的关键角色。这一新发现使该代谢通路有望成为 预防和治疗动脉断样硬化的一个免疫调节靶点。

在第五章中, 我们建立了一个高通量平台 (MacroScreen) 并以此采集数据来评估心 血管炎症背景下巨噬细胞的功能变化。通过将采集到的描述人类巨噬细胞应对各种 刺激的功能性改变的数据集与巨噬细胞转录组数据进行联合分析, 我们呈现出巨噬 细胞在功能层面和转录层面应对不同环境和刺激下的可塑性。该数据以及平台可以 作为未来研究巨噬细胞异质性的参考标准。 
利用这个平台, 在第六章中我们评估了急性心肌梗死患者发病后全身循环系统对血 液中巨噬细胞功能的重塑。在这一章中, 我们应用了机器学习模型来区分急性心肌 梗塞患者的血清和对照组血清对巨噬细胞功能的影响。随后, 我们将暴露于急性心 肌梗塞患者血清的巨噬细胞的功能组学数据与网络建模后的转录组数据进行整合分 析, 进而发现了与不良临床预后相关联的基因调控网络。最后, 通过网络建模驱动 的计算药物笁选, 我们发现前列腺素 E2 信号通路是干预心肌梗死不良预后发生的潜 在目标。

在第七章中, 我们证实动脉码样硬化斑块表征的性别特异性差异对最新发现的人类 动脉码样硬化斑块的转录组下的性别二态性有着显著的贡献。这一信息对于未来心 血管疾病的研究以及设计针对性别差异的精准医疗方案提供了非常关键的参考信息。

最后, 在第八章中, 我们探讨了本论文的主要发现和潜在的应用前景, 以及在生物 医学研究中应用计算机分析的几个概念性问题。

综上所述, 通过应用计算驱动分析, 包括统计推断、机器学习、多组学联合分析和 网络建模等, 于人类动脉粥样硬化组织和血液的多组学数据, 我们以系统和全面的 角度在分子水平上对心血管疾病进行了深度建模和分析。这些计算和数据驱动的分 析方法为了解疾病机制, 发现新的治疗靶点和药物以用于治疗动脉硬化提供了全新 的见解和启示。 


Chapter 10

Impact 



\section{SCIENTIFIC IMPACT}

\section{Systematic depiction of molecular processes in atherosclerosis}

Atherosclerosis is a chronic inflammatory disease hallmarked by a series of complex biological processes including intravascular lipid accumulation, innate and adaptive immune responses, and angiogenesis and the dysfunction of vascular smooth muscle cells ${ }^{1-3}$. Even though tremendous efforts have been made to study the mechanisms of atherogenesis, a systematic depiction of the formation and progression of atherosclerosis at a molecular level is still lacking. These insights are required for better understanding the disease progression, and the identification of therapeutic targets and the design of new drugs.

Biological entities, such as genes, proteins and metabolites, function as a system. With the recent progress in systems biology and computational biology, as well as the wide application of biological big-data collection, constructing a systematic disease model for atherosclerosis is becoming realistic. In this thesis, we demonstrated the advantages and perspectives of a systems biology approach to the study of atherosclerosis, as compared to a single focus. Specifically, through gene co-expression clustering followed by directional, causal network analysis, in chapter 2 we revealed the interaction of key atherogenic processes, and pinpointed the pivotal role of T cell activities in atherosclerosis. In chapter 4 , we constructed a metabolic model of the atherosclerotic plaque based on the mRNA expression profile of this tissue. To our knowledge, this is the first metabolic atlas for human atherosclerosis. Further analysis revealed metabolic key changes in atherosclerotic disease progression, including in glutamate/glutamine metabolism, and was able to link this to compromised macrophage function. This pathway could serve as a novel therapeutic target for the disease. However, the established plaque metabolic atlas is more versatile and represents a referential metabolic map of atherosclerosis to the cardiovascular research field, facilitating further explorations of disease-associated metabolites and reactions by the other researchers.

\section{Seeking innovations through interdisciplinary collaboration}

Innovations often emerge at the intersection of multiple disciplines. As a PhD candidate with a background in computer science and bioinformatics, I have been working in this biomedical group and collaborating with biologists throughout my $\mathrm{PhD}$ career. I received substantial supports in biological content from the vascular pathology group, meanwhile, my colleagues and biologists in collaboration have been influenced by the opportunities and power of computational strategies to biomedical research. The close collaboration between biologists and computer scientists eventually led to the discovery of novel findings, which could not have been obtained on the basis of a single discipline. 
The major part of this thesis aims at solving biological research questions using computational-driven biological data analysis, including machine learning, biological network modelling and multimodal data integration. These methods and analyses allow 1 ) extensive screening of valid targets from large data; 2) revealing hidden knowledge and associations underlying biological big-data; and 3) reconstruction of the whole biological system, to levels far beyond what conventional biomedical approaches could achieve. In this thesis, these computational-driven methods play essential roles in discovering new drug targets and providing novel insights into the disease mechanisms, creating unprecedented value (for example, by creating a metabolic map of human carotid plaque) across boundaries between computer science and biomedicine. Moreover, to address biomedical research questions, this thesis goes one step further, by harmonising and fusing data from biological experimentation, biological public databases, and omics analyses with help of advanced computer algorithms. Our studies inspire biologists to keep an open mind in tracking and deploying cutting-edge informatics and computational technology in biomedical research, and encourage more and more computer scientists to enter life science and develop algorithms for scientific and realistic biological questions. 


\section{SOCIAL IMPACT}

\section{Novel therapeutic targets and drugs for cardiovascular diseases}

Cardiovascular diseases, which are mainly driven by atherosclerosis, remain one of the leading causes of death worldwide ${ }^{4}$. It is estimated the CVD-related public healthcare expenditures will continuously rising, appealing to the urgent requirement for the discovery of new therapeutic targets and strategies for CVDs.

This thesis brings forward several potential therapeutic targets and drugs, as discussed in chapter 8. Current antiatherosclerotic therapies focus on the control of cardiovascular risk factors, such as dyslipidaemia, hypertension, and diabetes. However, a growing number of studies raised interest in targeting diseaseassociated molecular dysfunctions in plaque key cell types including $T$ cells, macrophages and smooth muscle cells ${ }^{5}$. The therapeutic targets that were identified in our studies all belong to the latter, and may hold promise for clinical application.

It should be noted that, in this thesis, we mainly relied on computational and network-based modelling of the atherosclerotic plaque and on machine learning algorithms for target discovery and drug screening. On the one hand, we made use of the publicly available omics data as well as biological pathway and drug target databases, which allowed establishing a comprehensive disease model while saving expenditures for unnecessary and redundant experiments. On the other hand, the extensive application of in silico analyses provided a more targeted approach to guide the subsequent biological exploration, which streamlines the following wetlab experiments, reduces the costs of the experiments and, in the end, of novel target identification and drug design. Compared with the conventional timeconsuming and costly design and development of new drugs, in silico screening and repurposing of "old" drugs that are approved by the U.S. Food and Drug Administration (FDA) or the European Medicines Agency (EMA) is much faster, as these drugs have already proved to be safe in clinical trials. This important feature of in silico data-driven drug screening and repurposing can help to significantly reduce the costs for drug development and shorten the developing timelines ${ }^{6}$.

Based on network-guided in silico drug screening and repurposing, in chapter 2 we identified epidermal growth factor receptor (EGFR) inhibitors as candidate drugs, targeting adverse $T$ cell functions related to plaque stability. To translate our findings into clinical application, we will perform ex vivo experiments to test the effect of these drugs on the human plaque. Further in vivo experiments and clinical trials would need to verify the validity of the candidate drugs; however, the safety of these drugs has been tested, which may simplify the trial design and accelerate the landing on the ground. Moreover, in chapter 4 we proposed the central role of 
macrophage glutamate/glutamine metabolism in plaque inflammation. Future experiments have been planned to validate the Glu/GIn metabolic pathways in vitro by inhibiting key enzymes in this pathway, such as glutamine synthetase (GLUL), in human macrophage culture, and evaluating the functional changes of GLUL inhibition through the macrophage functionomics platform we have established (see chapter 5 ). These novel therapeutic solutions could add to the current lipidlowing strategy, especially in cardiovascular patients that are insufficiently responding to the latter therapy. 


\section{CONCLUSIONS}

In conclusion, this thesis attempts to explore atherosclerotic disease by systemsbased computational approaches, in order to identify novel targets and drugs for therapeutic intervention. These findings may bring new conceptions for the pathogenesis, but also for the prevention or treatment of this disease, which could benefit the patients and reduce the socio-economic burden of CVD. But more importantly, it illustrates the merits of the employed systems-based methodology to study atherosclerosis, and may encourage further and more advanced applications of computational-driven big-data analysis in biomedicine research. 


\section{REFERENCES}

1. Bentzon JF, Otsuka F, Virmani R, Falk E. Mechanisms of plaque formation and rupture. Circ Res. 2014;114(12):1852-1866.

2. Shah PK. Mechanisms of plaque vulnerability and rupture. J Am Coll Cardiol. 2003;41(4 SUPPL. 4):S15-S22.

3. Tabas I, Lichtman AH. Monocyte-Macrophages and T Cells in Atherosclerosis. Immunity. 2017;47(4):621-634.

4. Virani SS, Alonso A, Benjamin EJ, et al. Heart disease and stroke statistics2020 update: A report from the American Heart Association. Circulation. 2020;141:E139-E596.

5. Libby P, Everett BM. Novel Antiatherosclerotic Therapies. Arterioscler Thromb Vasc Biol. 2019;39(4):538-545.

6. Pushpakom S, lorio F, Eyers PA, et al. Drug repurposing: Progress, challenges and recommendations. Nat Rev Drug Discov. 2018;18(1):41-58. 




\section{Appendices}

List of abbreviations 



\begin{tabular}{|c|c|}
\hline$\alpha \mathrm{SMA}$ & Alpha smooth muscle actin \\
\hline AAA & Amino acid analysis \\
\hline $\mathrm{ACN}$ & Acetonitrile \\
\hline $\mathrm{AHA}$ & American Heart Association \\
\hline AKG & $\alpha$-Ketoglutarate \\
\hline AMI & Acute myocardial infarction \\
\hline ANN & Artificial neural network \\
\hline ApoB & Apolipoprotein B \\
\hline ARACNe & Algorithm for the reconstruction of accurate cellular networks \\
\hline ARG1 & Arginase 1 \\
\hline ASC & Apoptosis-associated speck like \\
\hline ATAC & Assay for transposase accessible chromatin \\
\hline ATP & Adenosine triphosphate \\
\hline AUC & Area under the curve \\
\hline BER & Balanced error rate \\
\hline BiKE & Biobank of Karolinska Endarterectomy \\
\hline BLVRB & Biliverdin reductase $B$ \\
\hline CAD & Coronary artery disease \\
\hline CANTOS & Canakinumab Anti-Inflammatory Thrombosis Outcome Study \\
\hline CAS & Chemical Abstracts Service \\
\hline CCA & Canonical correlation analysis \\
\hline CCM & Correlation coefficient matrix \\
\hline CD & Cluster of differentiation \\
\hline CEA & Carotid endarterectomy \\
\hline CES & Cluster enrichment score \\
\hline ChIP-seq & Chromatin immunoprecipitation followed by sequencing \\
\hline $\mathrm{Cl}$ & Confidence interval \\
\hline CITE-seq & Cellular indexing of transcriptomes and epitopes by sequencing \\
\hline CMR & Cardiac magnetic resonance \\
\hline CNV & Copy number variation \\
\hline CTMM & Centre for Translational Molecular Medicine \\
\hline CVD & Cardiovascular Disease \\
\hline CyTOF & Mass cytometry \\
\hline DCFDA & $2^{\prime}, 7^{\prime}$-dichlorofluorescin diacetate \\
\hline DEG & Differentially expressed gene \\
\hline DIABLO & $\begin{array}{l}\text { Data integration analysis for biomarker discovery using latent } \\
\text { components }\end{array}$ \\
\hline DNN & Deep neural network \\
\hline DPI & Data processing inequality \\
\hline DTT & Dithiothreitol \\
\hline EC & Endothelial cell \\
\hline
\end{tabular}




\begin{tabular}{|c|c|}
\hline ECG & Electrocardiogram \\
\hline ECM & Extracellular matrix \\
\hline EDV & End-diastolic volume \\
\hline EF & Ejection fraction \\
\hline EGFR & Epidermal growth factor receptor \\
\hline ELISA & Enzyme-linked immunosorbent assay \\
\hline ELK4 & ETS transcription factor ELK4 \\
\hline EMA & European Medicines Agency \\
\hline ER & Endoplasmic reticulum \\
\hline FACS & Fluorescence-activated cell sorting \\
\hline FDA & U.S. Food and Drug Administration \\
\hline FEA & Femoral endarterectomy \\
\hline GC & Glucocorticoids \\
\hline GENIE3 & Gene network inference with ensemble of trees \\
\hline GEO & Gene expression omnibus \\
\hline Gln & Glutamine \\
\hline GLS & Glutaminase \\
\hline Glu & Glutamate \\
\hline GLUL & Glutamate-ammonia ligase \\
\hline GM-CSF & Granulocyte-macrophage colony-stimulating factor \\
\hline GO & Gene ontology \\
\hline GOBP & Gene ontology biological process \\
\hline GOMF & Gene ontology molecular function \\
\hline GOCC & Gene ontology cellular component \\
\hline GSEA & Gene set enrichment analysis \\
\hline GSOA & Gene set overrepresentation analysis \\
\hline GWAS & Genome-wide association study \\
\hline GEM & Genome-scale metabolic model \\
\hline GRN & Gene regulatory network \\
\hline$H \& E$ & Haematoxylin and eosin \\
\hline HCA & High content analysis \\
\hline HDL & High-density lipoprotein \\
\hline $\mathrm{HF}$ & Heart failure \\
\hline HGNC & HOGO Gene Nomenclature Committee \\
\hline HMR & Human metabolic reaction \\
\hline HPLC & High-performance liquid chromatography \\
\hline IC & Immune complexes \\
\hline IFN & Interferon \\
\hline IHD & Ischemic heart disease \\
\hline IL & Interleukin \\
\hline INFSIZE & Infarct size \\
\hline
\end{tabular}




\begin{tabular}{|c|c|}
\hline iNOS & Inducible nitric oxide synthase \\
\hline $\mathrm{IPH}$ & Intraplaque haemorrhage \\
\hline IRF7 & Interferon regulatory factor 7 \\
\hline ITRAQ & Isobaric tag for relative and absolute quantitation \\
\hline KEGG & Kyoto Encyclopedia of Genes and Genomes \\
\hline k-NN & k-nearest neighbours \\
\hline LA & Lauric acid \\
\hline LASSO & Least absolute shrinkage and selection operator \\
\hline LC-MS & Liquid chromatography-mass spectrometry \\
\hline LDL & Low-density lipoprotein \\
\hline LiA & Linoleic acid \\
\hline LINCS & Library of Integrated Network-based Cellular Signatures \\
\hline LPS & Lipopolysaccharide \\
\hline LXRB & Liver $X$ receptor beta \\
\hline MaasHPS & Maastricht Human Plaque Study \\
\hline MALDI & Matrix-assisted laser desorption ionization \\
\hline M-CSF & Macrophage colony-stimulating factor \\
\hline MFAP4 & Microfibril associated protein 4 \\
\hline MI & Myocardial infarction \\
\hline MIC & Maximal information coefficient \\
\hline MKL1/2 & Megakaryoblastic leukemia-1/2 \\
\hline MPTC & Maastricht pathology tissue collection \\
\hline mRMR & minimal-redundancy-maximal-relevance \\
\hline mRNA & messenger RNA \\
\hline MRTF & Myocardin-related transcription factor \\
\hline MS & Mass spectrometry \\
\hline MSigDB & Molecular Signatures Database \\
\hline MSO & Methionine sulfoximine \\
\hline MSTFA & N-methyl-N-(trimethylsilyl) trifluoroacetamide \\
\hline $\mathrm{NCBI}$ & National Center for Biotechnology Information \\
\hline NES & Normalized enrichment score \\
\hline NF-KB & Nuclear factor-kappa B \\
\hline NMOC & Non-mitochondrial oxygen consumption \\
\hline OA & Oleic acid \\
\hline oxLDL & Oxidized low-density lipoprotein \\
\hline P3C & Pam3CSK4 \\
\hline PA & Palmitic acid \\
\hline PBMC & Peripheral blood mononuclear cell \\
\hline PBS & Phosphate buffered saline \\
\hline PCA & Principal component analysis \\
\hline PCC & Pearson's correlation coefficient \\
\hline
\end{tabular}




\begin{tabular}{ll}
\hline PCI & Percutaneous coronary intervention \\
PCSK9 & Proprotein convertase subtilisin/kexin type 9 \\
PDGFR & Platelet-derived growth factor receptor alpha \\
PFA & Paraformaldehyde \\
PFAS & Phosphoribosylformylglycinamidine synthase \\
PGE2 & Prostaglandine E2 \\
PPAR- $y$ & Peroxisome proliferator-activated receptor gamma \\
PPI & Protein-protein interaction \\
PRDM1 & PR domain zinc finger protein 1 \\
PWM & Position weight matrix \\
QC & Quality control \\
RAAS & Renin-angiotensin-aldosterone system \\
RF & Random forest \\
RIN & RNA integrity number \\
RNA-seq & RNA sequencing \\
ROC & Receiver operating characteristic \\
ROS & reactive oxygen species \\
RSN & Robust spline normalization \\
RUNX3 & RUNX family transcription factor 3 \\
RAR- $\alpha$ & Retinoic acid receptor alpha \\
RAR- - & Retinoic acid receptor gamma \\
SA & Stearic acid \\
SCRNA-seq & single-cell RNA sequencing \\
SCX & Strong cation exchange \\
SD & Standard deviation \\
SEM & Standard error of the mean \\
SHAP & Shapley additive explanations \\
SLC25A12/13 & Solute carrier family 25 member 12/13 \\
SLPS & Standard lipopolysaccharide \\
SMAD & Small mothers against decapentaplegic \\
SMC & Smooth muscle cell \\
SNP & Single-nucleotide polymorphism \\
SPLS-DA & sparse partial least squares -discriminant analysis \\
SRF & Serum response factor \\
STARNET & Stockholm-Tartu Atherosclerosis Reverse Networks Engineering \\
& Tasks \\
STEMI & ST-elevation myocardial infarction \\
SVM & Support vector machine \\
TCA cycle & Tricarboxylic acid cycle \\
TCF21 & Transcription factor 21 \\
TCGA & The Cancer Genome Atlas \\
Page I 270 & \\
& \\
&
\end{tabular}




$\begin{array}{ll}\text { TEAB } & \text { Triethylammonium bicarbonate } \\ \text { TF } & \text { Transcription factor } \\ \text { TFA } & \text { Trifluoroacetic acid } \\ \text { TGF } & \text { Transforming growth factor beta } \\ \text { Th } & \text { Helper T cell } \\ \text { tINIT } & \text { task-driven integrative network inference for tissues } \\ \text { TNF } & \text { Tumour necrosis factor } \\ \text { TOM } & \text { Topological overlap matrix } \\ \text { TPM } & \text { Transcripts per million } \\ \text { TPP } & \text { TNF }+ \text { PGE2 + P3C } \\ \text { Treg } & \text { Regulatory T cell } \\ \text { tSNE } & \text { t-distributed stochastic neighbour embedding } \\ \text { TSS } & \text { Transcription start site } \\ \text { UMAP } & \text { Uniform manifold approximation and projection } \\ \text { upLPS } & \text { Ultra-pure lipopolysaccharide } \\ \text { VEGF(R) } & \text { Vascular endothelial growth factor (receptor) } \\ \text { WHO } & \text { World health Organization } \\ \text { WGCNA } & \text { Weighted gene co-expression network analysis } \\ \text { XGB } & \text { XGBoost }\end{array}$





\section{Appendices}

Acknowledgements | 致谢 

First, I would like to acknowledge the funding I received from the China Scholarship Council (CSC) to support my PhD study.

I would also like to thank the members of my thesis assessment committee: Prof. dr. Ilja Arts (Chair), Prof. dr. Menno de Winther, Prof. dr. Yvan Saeys, and Dr. S.L.M. Steinbusch - Coort, for the time and effort you spent on reading and evaluating my thesis.

Next, I would like to express my sincere gratitude to my supervision team.

Prof. dr. Erik A.L. Biessen, you are the only one who supervised all my 5-year PhD study, acting as the promoter, the supervisor, and the daily supervisor. Five years have passed, I can still remember the skype interview we had, and the first day we met in the corridor at the MUMC. That is where my PhD started. Thank you for offering me this great opportunity to join our group and bringing me into cardiovascular research. To be honest, I was totally unconfident with my PhD study at the beginning. But now, with the nourishment of five-year PhD experiences in our group, I am certain to say I grow up outstandingly, and more importantly, I realize that I do like our research.

The knowledge and research are not the only things I gain under your supervision. Your enthusiasm for applying state-of-the-art technology and bold attempts in doing research encourage me to pursue endless progress and further development. You also tell me that never underestimating my ability and effort. I will always follow your edification and hope to make you proud of me. Besides, I also learn from you how to cultivate PhD students, and how to manage a research team. These experiences will be valuable treasures in my future academic career.

Prof. dr. Judith C. Sluimer, standing at the end of my PhD, I could not imagine how things will go if I work under your supervision earlier. Despite the short format, it is still unbelievable how fast we made our research letter in only one month, from just an idea to submission. What if we started earlier? In my first or second year? Then we probably will have one letter per month, and finally get 60 letters! Just kidding... : ) Of course, we were not born for letters, but for big projects. Your plaque fibroblast data and study design make me feel very surprised! It must be a magnificent project and I am pretty sure it will eventually be a milestone in the vascular fibroblast field. If we start earlier or I stay longer, we must have a lot of cool single-cell sequencing things!

I feel very enjoyable with my work and research under your supervision. You are always kind and patient, tolerating my stupid questions and never get bored. But these are not all my impressions of you. You always speak super-fast; your fast pace with high heel sound often distracts me from my work and tensions me a lot; your tons of typos in emails and manuscript comments make me confused for thousand 
times... Luckily, I get survived, and I have imparted my experiences to Baixue with a book How to work with Judith!

Dr. Evgueni Smirnov, the most reliable computer scientist in my PhD study, thank you for all your efforts and inputs on my PhD study. You always come the first to help me to solve mathematical problems. I have a local Dutch phone number, but you are the only one who called me in these five years. This is why I was a bit confused when I picked up the phone, did you feel that? Also, thanks for organising the symposium COPA 2018. This is my first time to see a talk by a top scientist, Vladimir Vapnik, which I only read in a textbook before.

I would also like to thank two mentors for acting key roles in my PhD study, Dr. Lieve Temmerman and Dr. Joël M.H. Karel.

Lieve, you probably do not remember the first presentation I made in our lab meeting. It was just one month after I joined the group. And frankly, I am an extremely nervous guy, with little experience in giving a presentation, not to mention how to make it fully and fluently in English. This made my first lab meeting horrible. But I remember, for every sentence I said, I can always read an exaggerated expression and action for confirmation from your face. Five years later, if I look back, I guess at that time you did not understand what I was talking about at all, and your actions for confirmation were so dramatic. But these are very important to me - without your responses and encouragement, I probably could not continue my first lab meeting presentation. Thank you for your encouragement, thank you for being patient with our collaborative studies, and thank you for your adaption to working with a bioinformatician with a mountain of data and results. These two are the most important studies in my PhD.

Joël, you always use the simplest case to explain the most complicated theory. Every time I ask mathematical questions, it always makes me feel I am talking with the best tutor in the world. You must be a very good teacher and tutor at DKE. You put a lot of time and effort into my PhD studies, and I hope you are satisfied with my achievements now. Thank you for all your supports in the past five years.

Then, I would like to thank the people with whom I collaborated in my PhD. Dr. Ljubica Matic from Karolinska Institute, thank you for your endless supports of my first paper. We will become neighbours after I moving to Solna, I wish we will have more collaborations and be more productive in future. Prof. dr. Adil Mardinoglu and Dr. Cheng Zhang from the King's College London and the SciLifeLab, thank you for all your supports to our joint study. I believe our collaborative study will come to a very interesting and impactful paper in cardiovascular research. Moreover, I am looking forward to my postdoc at SciLifeLab! Dr. Thomas Ulas and Marie Oestreich from the University of Bonn, it is my honour to work with you two. Thank 
you for your patience and all the R scripts and discussions we had for our study.

Now the guys in our lab. Margaux, I always think if you stay longer, we can do more exciting things. I believe our collaborative studies will be the highlights in my PhD. For this, I will try my best to publish our studies. By the way, your French-speaking is very pleasant to me, despite I understand nothing in your language. I expect to hear your voice in future! Jan, the one who started PhD at the same time! Thank you for being responsible for our collaborative studies. I wish you all the best with your PhD and more importantly, the great job and peaceful life you will have, no matter in your hometown or somewhere afterwards. Pieter, thank you for paying me for my first lunch meal in MUMC, and also for your great help in our study. Wish you together with Chang have more exciting outcomes published in future! Kim, the OG of our PhDs! Thank you for all our collaborations in the past, also for all your instructions on my PhD thesis and defense preparation. I hope you like my gift for you, from an oriental country. Chang, I hope you enjoy your life and study here. Take easy with everything. Sometimes things you expected will come true when you no longer have eager expectations. Good luck with your PhD and I hope one year after you will get a good position for your further development. Adele, when I met you the first time (I assumed I was trying to fix your computer) I did not realize you will be as brilliant as you are today. Your chariness and responsibility are the noble characteristics that I am lacking. The only thing I feel regret is that we do not have a joint project to work together, and I strongly wish we could have more opportunities for collaboration in future. Renée, thank you for your greeting card and kind words! In my eye you are a very reliable mate and work very hard, that is why I told Baixue that she should learn as much as from you. Of course, you will be a very good mentor and researcher! Elias, I wish you could join our group earlier, then we will make street photography together and solidify many memories in Maastricht. You must show me how to develop films before I leave. This is the thing staying in my mind for many years when I started using film cameras. We can make it, right? Olivia, you don't know how much I want to work with you. With your help, I never worry about my manuscript writing. I always think about how I feel if someone asks me for language checking in Chinese. It must be a wonderful experience, don't you think? Sebastiaan, hey guy, special thanks to you "for bringing my shape back"! Also for the thesis, without you, I cannot make my Dutch summary, which makes this thesis look great! I wish one day your plan will become true, you speak fluence Chinese and get you onboard this fast-developing train. By then I will run an affiliated company with you in China! Laura, Valeria, Mat, Gregorio, Marjo, Axel, Dietbert, Marion, Jenny, Jasper, Ruud, Anke, thank you all for your help and supports in my PhD. I wish you all the best in future!

Besides, I would like to thank the people from Helmholtz Centre for Infection Research (HZI) in Braunschweig. Prof. dr. Alice McHardy, thank you for offering me 
the opportunity to study in Germany, as well as your tolerance and patience in my work. I feel regret that I did not make a substantial contribution to your group, however, I hope you will be satisfied with my progress and will felicitate for the opportunity you offered to me when you receive this thesis. Dr. Aaron Weimann, thank you for being my mentor, I hope all the best for your future development. Gary, thanks for the time and moments we had in the past. Goslar is probably the smallest city I have ever visited. I hope someday in future we can meet again. My best wishes to your family and your little daughter! Thorsten and Jacqueline, it is great to meet and work with you, and I wish you all the best in Germany.

To my Chinese friends and colleagues in Maastricht, it is my great pleasure to meet you, to work and enjoy our life together in a foreign country. 很荣幸在马城能认识 赵俊芳师兄和李婕一师姐。我对你们在祖国抗疫一线舍身忘我的奉献精神感到由衰 钦佩, 我为我有你们这样的师兄师姐感到骄傲。祝你们在武汉一切都好, 也祝你们 的小宝贝健康茁壮成长。张明师姐, 很希望有一天能到海口去看你, 我们一起撸串, 烤海鲜, 吃榴莲, 看海, 顺便再让张教授耳提面命面授机宜一番, 提升一下我的科 研素养和觉悟。祝愿兵哥最后一年在比利时的学业顺利, 这样我们四个就可以早日 在海南团聚。张硕师兄, 在我写下这些文字的时候, 距离你答辩就只有三天了。祝 贺你终于走完了你人生中在马城的这一段路。我想, 不论未来你选择哪条路都不会 错, 而且我预感我们回国以后应该相距不远, 希望我们有一天仍然有机会在夕阳下 坐在河边喝着啤酒聊着天。乔奇, 你的博士生涯, 在 2020 年的那个初秋就这样干脆 利落地结束了。你回国以后我时常在想, 也许马城和荷兰的这段经历在你生命中就 像是一场梦一样, 如小桥流水一般涓涓流淌而过, 在你回国后就永远变成了遥远的 记忆, 似乎自己曾经来到过这么一个地方, 有过这么一段经历, 然后梦醒了, 结束 了, 便似乎真的不存在了。希望你在郑州和你先生经营好自己的事业和家庭, 过着 属于你的平和安心的生活。傲敏, 依稀记得你我和乔奇在我们刚到马城的艰难岁月 中互相帮助，扶持和鼓励。希望荷兰的这段经历让你心中最初的那个梦想生根发芽， 长成参天大树。也许你现在的工作仍然面临着很大的挑战, 无论如何, 希望你在北 京一切都好, 也希望我们回国以后还能再聚。此外还要感恩结识的除医学院以外其 他学院的同学。爽姐, 遗憾没办法去上海和你做同事了。真的非常佩服你的能力和 敢想敢干的风格, 希望你在上海一切都好, 我们来日再聚。溪溪, 其实我们没来马 城就已经加微信了, 对吗? 也许我比法学院的同学认识你都早。谢谢你帮我改签, 不然我真的要跑到韩国去了。真的很佩服你知识面的广阔, 还有办事的沉稳周密, 你是我在这边认识的同学里最靠谱的人, 你有这样的能力无论到哪里都会发光的。 骁哥, 我们以后还有机会一起自驾游吗? 不要忘了我们曾经在艳阳高照的南法一路 前行, 在普罗旺斯看到的花海, 在阿维尼翁吃过的晚餐, 在马赛, 尼斯, 摩纳哥, 红土城, 塞南克修道院走过的每一步, 看过的每一个风景, 还有在那北极圈的冰天 雪地里一起謷过的漫漫长夜和追寻过的极光。海洋, 谦谦, 宋柯, 感恩我们在马城 所经历的一切美好时光。特别是海洋, 临走之前无论如何咱俩还得再干一瓶威士忌。 这里特别要感谢欣欣, 如果刚来就认识你就好了, 那我们这些年一定会留下很多欢 乐和美好的回忆。回想起来, 在不认识你的时候, 我就在东方行门口见过你在那里 
展示中国茶艺文化呢。真的很佩服你工作上的开疆拓土、披荆斩棘的能力。如果我 有一天回到马城我一定第一时间联系你, 也希望有朝一日我们可以在大连的天津街 上一起吃烤生蚝。

此外, 我还想感谢我的上司刘亦薇女士对我出国留学以及在选择人生道路上做出过 的帮助和指引。如果没有当初在贵公司的经历, 就不会有今天的我。也许当初我们 看不到那么长远, 然而回顾过去的经历我无意间发现, 您竟然是在我人生中最具决 定性的两个节点出现的关键 NPC。在您一直以来的关照下, 让一个爱玩游戏的我找 到了人生的航向, 完成了求学之路上中的两次重大跨越。遗憾的是没能在布达佩斯 见到您。说真的, 老于我们都在布鲁塞尔见了, 咱怎么就没见到呢? 不过我想以后 还有机会的。另外我还要特别感谢一下画家李文阿姨, 您亲自创作的封面给这本学 术的论文增添了艺术的色彩。看着您的作品, 仿佛又让我重走了一遍卢浮宫, 奥赛, 泰特, 普拉多……回顾了我在欧洲看过的所有艺术作品, 再次感受了一遍这些艺术作 品中源源不断涌现出的灵感, 以及曾经在那个城市漂泊着的心情。

最后, 我想把我最诚挚的感谢留给我的家人。感谢我的父母, 为我创造了一个宽松 的环境让我能够无所顾忌地去追寻自己的梦想。在外留学最挂念不下的就是父母。 希望这些年我没有㙜负您们对我的期望，希望您们会为我感到骄傲，更希望以后能 有更多的时间去陪伴您们，去弥补这些年没能留在您们身边的遗憾。最后最后，我 要感谢我的女朋友司全乐博士，是你在身边伴我走过了这人生中最不寻常、最值得 回忆的五年。你的精心照顾扫清了我的后顾之忧, 让我可以全身心地投入到学术当 中。你的耐心和包容让我变得更加成熟。我们已经一起走过二十多个国家了，从撒 哈拉沙漠到地中海, 从东非大草原到北极圈, 还有更多的地方等着我们去征服。感 谢能在马城遇到你, 这是我在欧洲求学之路上最意外的收获。 



\section{Appendices}

Curriculum Vitae 



\section{About the Author}

Han Jin was born on the $31^{\text {st }}$ of January, 1987 in Tianjin, China, a coastal city that has a similar scale of population (15 million) as in the Netherlands. He was a crazy fan of video games and spent most of his childhood and teenage leisure time playing video games. During his high school study, he raised interests in computer programming and took part in the Olympiad competition in Informatics in China, which confirmed his talent and passion for computer science. From 2005 to 2009, he studied computer science for his bachelor's degree at Tianjin University of Science and Technology, after which he worked in a mobile game company as a programmer aiming at developing the world's best game. Tetris is the first game he developed, which worked very well on his Motorola mobile phone. His talent caught the eye of his boss, therefore he was encouraged by the company and his parents for further development. In 2011, he started his master's study at Nankai University, focusing on machine learning, data mining and bioinformatics. Having finished his master's study, in 2014 he left his hometown and mother country, to a faraway place, Braunschweig, Germany, and worked at the Helmholtz Centre for Infection Research (HZI) for an internship for one year. This valuable experience helped him to obtain his PhD position at Maastricht University, the Netherlands, specializing in computational biology in cardiovascular research. His PhD study started in 2016, and now it is coming to the end. In October 2021, he will continue his story in the EU as a postdoctoral researcher at the Science for Life Laboratory (SciLifeLab) and the KTH Royal Institute of Technology in Stockholm, Sweden. 
Personal Information

Name:

Date of birth:

Place of birth:

Nationality:

E-mail:

\section{Education}

Han Jin

$31^{\text {st }}$ of January, 1987

Tianjin, China

Chinese

hjin.unimaas@outlook.com

\section{PhD student}

Computational Biology in Cardiovascular Diseases

Department of Pathology, Maastricht University

Medical Center+ (MUMC+), Maastricht, The

Netherlands

Promoters: Prof. dr. Erik A.L. Biessen

Prof. dr. Judith C. Sluimer

Co-promoter: dr. Evgueni Smirnov

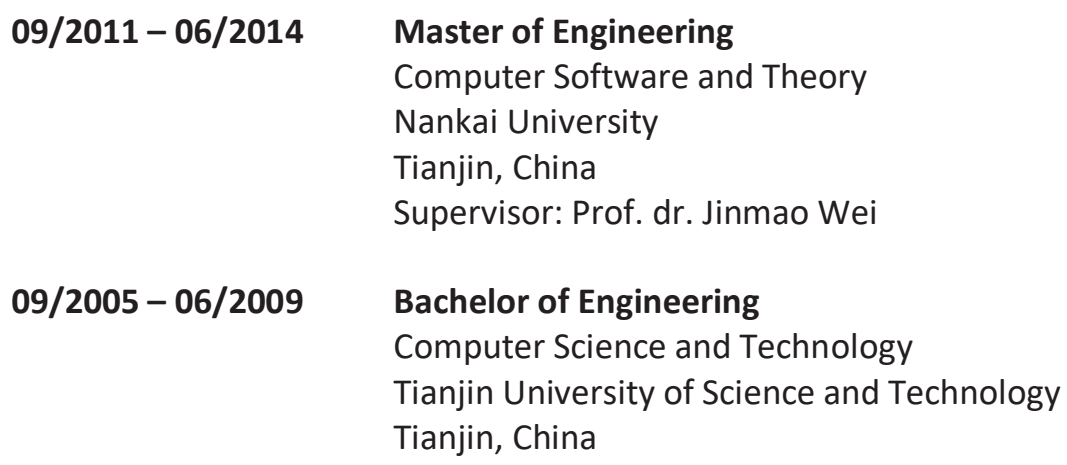

Scientific Visiting and Internship

$\begin{array}{cl}\mathbf{0 8 / 2 0 1 9 - 0 8 / 2 0 1 9} & \begin{array}{l}\text { Visiting scholar at Life and Medical Sciences Institute } \\ \text { (LIMES) } \\ \text { University of Bonn, Bonn, Germany } \\ \text { Supervisor: Dr. Thomas Ulas }\end{array} \\ \mathbf{0 8 / 2 0 1 8 - 0 8 / 2 0 1 8} & \begin{array}{l}\text { Visiting scholar at Science for Life Laboratory } \\ \text { (SciLifeLab) } \\ \text { KTH Royal Institute of Technology, Stockholm, Sweden } \\ \text { Supervisors: Prof. dr. Adil Mardinoglu } \\ \text { Dr. Cheng Zhang }\end{array}\end{array}$


09/2014-09/2015 Internship at Helmholtz Centre for Infection Research

(HZI)

Braunschweig, Germany

Supervisor: Prof. dr. Alice Carolyn McHardy

\section{Publications}

Integrative multi-omics analysis of human atherosclerosis reveals a serum response factor-driven network associated with intraplaque haemorrhage Han Jin, Pieter Goossens, Wouter Eijgelaar, Marco Manca, Joël Karel, Evgueni Smirnov, Cornelis J.J.M. Sikkink, Barend M.E. Mees, Olivia Waring, Kim van Kuijk, Gregorio Fazzi, Marion Gijbels, Martina Kutmon, Chris T.A. Evelo, Ulf Hedin, Judith C. Sluimer, Mat J.A.P. Daemen, Ljubica Matic, Erik A.L. Biessen Clinical and Translational Medicine, 2021

Deficiency of myeloid PHD proteins aggravates atherogenesis via macrophage apoptosis and paracrine fibrotic signalling

Kim van Kuijk, Jasper A F Demandt, Javier Perales-Patón, Thomas L Theelen, Christoph Kuppe, Elke Marsch, Jenny de Bruijn, Han Jin, Marion J Gijbels, Ljubica Matic, Barend M E Mees, Chris P M Reutelingsperger, Ulf Hedin, Erik A L Biessen, Peter Carmeliet, Andrew H Baker, Rafael K Kramann, Leon J Schurgers, Julio SaezRodriguez, Judith C Sluimer

Cardiovascular Research, 2021

The hypoxia-sensor carbonic anhydrase IX affects macrophage metabolism, but is not a suitable biomarker for human cardiovascular disease

J. A. F. Demandt, L. J. Dubois, K. van Kuijk, M. Zat'ovičová, H. Jin, S. Parkkila, S. W. van der Laan, L. Jelenska, B. M. E. Mees, C. P. M. Reutelingsperger, K. B. J. M. Cleutjens, C. J. H. van der Kallen, C. G. Schalkwijk, M. M. J. van Greevenbroek, E. A. L. Biessen, G. Pasterkamp, S. Pastoreková, C. D. A. Stehouwer, J. C. Sluimer Scientific Reports. 11.1 (2021): 1-14.

Low human and murine Mcl-1 expression leads to a pro-apoptotic plaque phenotype enriched in giant-cells

Margaux A. C. Fontaine, Marijke M. Westra, Ilze Bot, Han Jin, Aimée J. P. M. Franssen, Martine Bot, Saskia C. A. de Jager, Ivan Dzhagalov, You-Wen He, Bart J. M. van Vlijmen, Marion J. J. Gijbels, Chris P. Reutelingsperger, Theo J. C. van Berkel, Judith C. Sluimer, Lieve Temmerman, Erik A. L. Biessen

Scientific Reports, 9.1 (2019): 1-11. 
Gene Selection Based on Supervised Vector Representation of Genes

Yu T, Gao F, Jin H, Wei J

In Pacific Rim International Conference on Artificial Intelligence 2014 Dec 1 (pp.

834-841).

Probabilistic confusion entropy for evaluating classifiers

Wang XN, Wei JM, Jin H, Yu G, Zhang HW

Entropy. 2013 Nov;15(11):4969-92.

Learning decision trees using confusion entropy

Jin H, Wang XN, Gao F, Li J, Wei JM

In 2013 International Conference on Machine Learning and Cybernetics $2013 \mathrm{Jul}$ 14 (Vol. 2, pp. 560-564).

Feature selection based on complementarity of feature classification capability Gao F, Yu T, Wei Y, Jin H, Wei JM

In 2013 International Conference on Machine Learning and Cybernetics $2013 \mathrm{Jul}$ 14 (Vol. 1, pp. 130-135).

A search log clustering algorithm based on the idea of hierarchy

Hou SS, Jin H, Wei Y, Wang X, Fan BQ, Wei JM

In 2013 International Conference on Machine Learning and Cybernetics $2013 \mathrm{Jul}$ 14 (Vol. 1, pp. 69-74).

Blood milieu in acute myocardial infarction reprograms human macrophages for trauma repair

Margaux A. C. Fontaine†, Han Jint, Mick Gagliardi, Mat Rousch, Erwin Wijnands, Monika Stoll, Leon Schurgers, Chris Reutelingsperger, Casper Schalkwijk, Nynke M. S. van den Akker, Daniel G.M. Molin, Lars Gullestad, Jan Eritsland, Pavel Hoffman, Mona Skjelland, Geir $\varnothing$. Andersen, Pål Aukrust, Joël M.H. Karel, Evgueni Smirnov, Bente Halvorsen, Lieve Temmermant, Erik A.L. Biessen†

+ Authors contributed equally

Submitted to Science Immunology

Transcriptional sex dimorphism in human atherosclerosis relates to plaque type

Han Jin, Barend M.E. Mees, Erik A.L. Biessen, Judith C. Sluimer

Submitted to Circulation Research, in revision 
Low density lipoprotein exposure of plasmacytoid dendritic cells blunts Tolllike receptor $7 / 9$ signaling via NUR77

Erik A.L. Biessen, Pieter Goossens, Erwin Wijnands, Han Jin, Bart Legein, Tammy Oth, Aaron Issace, Monica Stoll, Joris Vanderlocht, Mat J.A.P. Daemen, Martin Zenke, Anette Christ

Submitted to Journal of Lipid Research, in revision

Identification of a PRDM1-driven gene network to regulate $T$ cell activity/exhaustion in ruptured human plaque

Han Jin, Emiel van der Vorst, Sanne L. Maas, Chang Lu, Jan Nagenborg, Marco Manca, Joël M.H. Karel, Rachel Cavill, Olivia Waring, Cornelis J.J.M. Sikkink, Barend M.E. Mees, Mat J.A.P. Daemen, Evgueni Smirnov, Judith C. Sluimer, Erik A.L. Biessen In preparation

Genome-scale metabolic modelling of human carotid plaque reveals the pivotal role of glutamine/glutamate metabolism in plaque inflammation and vulnerability

Han Jin, Cheng Zhang, Jan Nagenborg, Peter Juhasz, Adele Ruder, Cornelis J.J.M. Sikkink, Barend M.E. Mees, Mat J.A.P. Daemen, Judith C. Sluimer, Dietbert Neumann, Pieter Goossens, Marjo Donners, Adil Mardinoglu, Erik A.L. Biessen In preparation

Towards a function-based model of macrophage plasticity: the MacroScreen platform

Margaux A.C. Fontaine, Han Jin, Marie Oestreich, Joram Huckriede, Pieter Goossens, Mat Rousch, Erwin Wijnands, Ludwig Dubois, MPH van de Waarenburg, Joachim Schultze, Judith C. Sluimer, Thomas Ulast, Lieve Temmerman†, Erik A.L. Biessen†

† Authors contributed equally

In preparation 
Conference Presentations

July 2021

$1^{\text {st }}$ Online EVBO/ESM (European Vascular Biology Organization and European Society of Microcirculation) Summer School

Presentation and Q\&A together with Prof. Judith Sluimer Human atherosclerosis transcriptomics and deep phenotyping yield new regulators of plaque (lymph)angiogenesis

November 2020 Maastricht School for Cardiovascular Diseases (CARIM) Symposium 2020. Maastricht, the Netherlands Selected postervideo presentation Network analyses on human plaque gene expression revealed pivotal roles of $T$ cells in atherosclerosis

May 2019

European Atherosclerosis Society (EAS) Meeting 2019. Maastricht, the Netherlands

Selected e-poster presentation

Integrative multi-omics analysis of human atherosclerosis reveals an intraplaque hemorrhage associated network, which offers better prediction of disease stage

March 2018

Applied Bioinformatics in Life Sciences ( $2^{\text {nd }}$ edition), Leuven, Belgium

Poster presentation

Integrative multi-omics analysis of human atherosclerosis reveals an intraplaque hemorrhage associated network

July 2013

International Conference on Machine Learning and Cybernetics, ICMLC 2013, Tianjin, China

Oral presentation

Learning decision trees using confusion entropy 

